Laurent Bartholdi · Markus Neuhauser · Wolfgang Woess

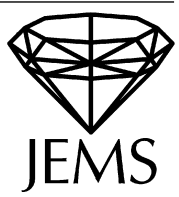

\title{
Horocyclic products of trees
}

Received February 1, 2006 and in revised form January 23, 2007

\begin{abstract}
Let $T_{1}, \ldots, T_{d}$ be homogeneous trees with degrees $q_{1}+1, \ldots, q_{d}+1 \geq 3$, respectively. For each tree, let $\mathfrak{h}: T_{j} \rightarrow \mathbb{Z}$ be the Busemann function with respect to a fixed boundary point (end). Its level sets are the horocycles. The horocyclic product of $T_{1}, \ldots, T_{d}$ is the graph $\operatorname{DL}\left(q_{1}, \ldots, q_{d}\right)$ consisting of all $d$-tuples $x_{1} \cdots x_{d} \in T_{1} \times \cdots \times T_{d}$ with $\mathfrak{h}\left(x_{1}\right)+\cdots+\mathfrak{h}\left(x_{d}\right)=0$, equipped with a natural neighbourhood relation. In the present paper, we explore the geometric, algebraic, analytic and probabilistic properties of these graphs and their isometry groups. If $d=2$ and $q_{1}=q_{2}=q$ then we obtain a Cayley graph of the lamplighter group (wreath product) $\mathfrak{Z}_{q} 2 \mathbb{Z}$. If $d=3$ and $q_{1}=$ $q_{2}=q_{3}=q$ then DL is a Cayley graph of a finitely presented group into which the lamplighter group embeds naturally. In general, when $d \geq 4$ and $q_{1}=\cdots=q_{d}=q$ is such that each prime power in the decomposition of $q$ is larger than $d-1$, we show that DL is a Cayley graph of a finitely presented group. This group is of type $F_{d-1}$, but not $F_{d}$. It is not automatic, but it is an automata group in most cases. On the other hand, when the $q_{j}$ do not all coincide, $\operatorname{DL}\left(q_{1}, \ldots, q_{d}\right)$ is a vertextransitive graph, but is not a Cayley graph of a finitely generated group. Indeed, it does not even admit a group action with finitely many orbits and finite point stabilizers. The $\ell^{2}$-spectrum of the "simple random walk" operator on $\mathrm{DL}$ is always pure point. When $d=2$, it is known explicitly from previous work, while for $d=3$ we compute it explicitly. Finally, we determine the Poisson boundary of a large class of group-invariant random walks on DL. It coincides with a part of the geometric boundary of DL.
\end{abstract}

Keywords. Restricted wreath product, trees, horocycles, Diestel-Leader graph, growth function, normal form, Markov operator, spectrum

\section{Contents}

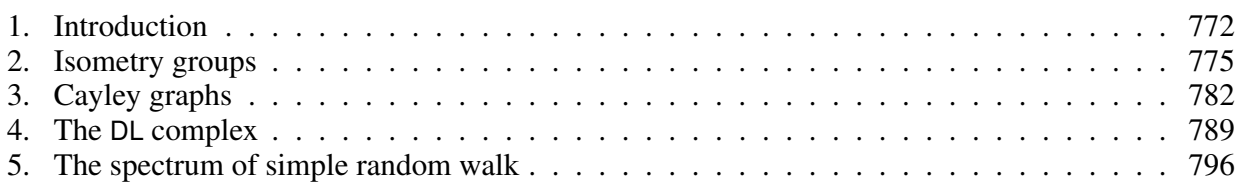

L. Bartholdi: École Polytechnique Fédérale de Lausanne (EPFL), Institut de Mathématiques B (IMB), 1015 Lausanne, Switzerland; e-mail: laurent.bartholdi@gmail.com

M. Neuhauser: Lehrstuhl A für Mathematik, RWTH Aachen, 52056 Aachen, Germany; e-mail: markus.neuhauser@matha.rwth-aachen.de

W. Woess: Institut für Mathematische Strukturtheorie (Math C), Technische Universität Graz, Steyrergasse 30, 8010 Graz, Austria; e-mail: woess@TUGraz.at

Mathematics Subject Classification (2000): 05C50, 20E22, 47A10, 60B15 
6. The Poisson boundary of a random walk . . . . . . . . . . . . . . . . . 808

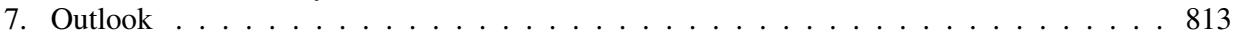

\section{Introduction}

Let $X$ be a locally finite, infinite, connected graph. We write $x \sim y$ if $x, y$ are neighbours (connected by an edge), and $\operatorname{deg}(x)$ for the number of neighbours of $x$. We assume that $X$ has no loops, that is, $x \ngtr x$ for all $x \in X$. Suppose that $X$ is written as a disjoint union of non-empty sets $H_{k}, k \in \mathbb{Z}$ (the horocycles), where each element in $H_{k}$ has neighbours both in $H_{k-1}$ and in $H_{k+1}$, but none in any other $H_{l}$. (This condition is tailored to our purposes and can be generalized.) The associated surjection $\mathfrak{h}: X \rightarrow \mathbb{Z}$, where $\mathfrak{h}(x)=k$ if $x \in H_{k}$, is a graph homomorphism of $X$ onto the two-way-infinite path $\mathbb{Z}$. We call it a Busemann function, although this terminology is justified completely only in specific cases (see below), and we say that $(X, \mathfrak{h})$ is a Busemann pair. Now let $X_{1}, \ldots, X_{d}$ be a family of such graphs with associated Busemann functions $\mathfrak{h}: X_{j} \rightarrow \mathbb{Z}$ (we use the same symbol $\mathfrak{h}$ for each of them). Then their horocyclic product is

$$
\prod_{j=1}^{d} \mathfrak{h} X_{j}=\left\{x_{1} \cdots x_{d} \in X_{1} \times \cdots \times X_{d}: \mathfrak{h}\left(x_{1}\right)+\cdots+\mathfrak{h}\left(x_{d}\right)=0\right\}
$$

with neighbourhood

$$
\begin{aligned}
& x_{1} \cdots x_{d} \sim y_{1} \cdots y_{d} \Leftrightarrow \\
& \text { there are } i \neq j \text { such that } x_{i} \sim y_{i}, x_{j} \sim y_{j} \text { and } x_{k}=y_{k} \text { for all } k \neq i, j .
\end{aligned}
$$

In particular, one must have $\mathfrak{h}\left(x_{i}\right)-\mathfrak{h}\left(y_{i}\right)=\mathfrak{h}\left(y_{j}\right)-\mathfrak{h}\left(x_{j}\right)= \pm 1$. Thus, $x=x_{1} \cdots x_{d} \mapsto$ $\mathfrak{H}(x)=\left(\mathfrak{h}\left(x_{1}\right), \ldots, \mathfrak{h}\left(x_{d}\right)\right)$ is a graph homomorphism of $\prod_{\mathfrak{h}} X_{j}$ onto the simplicial lattice $\mathbb{A}_{d-1}=\left\{\mathbf{k}=\left(k_{1}, \ldots, k_{d}\right) \in \mathbb{Z}^{d}: k_{1}+\cdots+k_{d}=0\right\}$. In that lattice, two points are neighbours if they differ by a vector $\mathbf{e}_{i}-\mathbf{e}_{j}$, where $i \neq j$ and $\mathbf{e}_{i} \in \mathbb{Z}^{d}$ is the unit vector with a 1 in its $i$-th coordinate.

There is an analogous construction for groups (cf. Kaimanovich and Woess [32, p. 356]). Let $\Gamma_{1}, \ldots, \Gamma_{d}$ be topological (e.g. in particular, discrete) groups, each one equipped with a continuous homomorphism $\mathfrak{h}: \Gamma_{j} \rightarrow \mathbb{Z}$ (or $\rightarrow \mathbb{R}$; we again use the same symbol $\mathfrak{h}$ for each of them). Then their horocyclic product is

$$
\prod_{j=1}^{d} \mathfrak{h} \Gamma_{j}=\left\{g_{1} \cdots g_{d} \in \Gamma_{1} \times \cdots \times \Gamma_{d}: \mathfrak{h}\left(g_{1}\right)+\cdots+\mathfrak{h}\left(g_{d}\right)=0\right\},
$$

which is a closed subgroup of the direct product of the $\Gamma_{j}$. For finitely generated groups $\Gamma_{j}$, this kind of construction was used previously by Bestvina and Brady [7]. However, our approach has a different "history", and below, the groups will be non-discrete isometry groups of homogeneous trees. Here, horocyclic products of groups will arise as isometry (automorphism) groups of horocyclic products of graphs. 
If $\left(X_{1}, \mathfrak{h}\right)$ and $\left(X_{2}, \mathfrak{h}\right)$ are two Busemann pairs, then a Busemann isometry $g$ from the former to the latter is a graph isomorphism $g: X_{1} \rightarrow X_{2}$ such that $x_{1} \mapsto \mathfrak{h}\left(g x_{1}\right)-\mathfrak{h}\left(x_{1}\right)$ is constant. We write $\mathfrak{h}(g)$ for this constant. The group Aut $(X, \mathfrak{h})$ of a given Busemann pair $(X, \mathfrak{h})$ consists of all Busemann isometries $X \rightarrow X$. Given $\left(X_{j}, \mathfrak{h}\right)$ as above $(j=$ $1, \ldots, d)$, the group $\prod_{j=1}^{d} \mathfrak{h} \operatorname{Aut}\left(X_{j}, \mathfrak{h}\right)$ acts on $\prod_{j=1}^{d} \mathfrak{h} X_{j}$ by graph isometries via

$$
g x=\left(g_{1} x_{1}\right) \cdots\left(g_{d} x_{d}\right), \quad \text { where } \quad g=g_{1} \cdots g_{d} \quad \text { and } \quad x=x_{1} \cdots x_{d}
$$

There are many examples of Busemann pairs, as well as generalizations of the definition. Some of them will be outlined in the final $\$ 7$.

The basic example of a Busemann pair arises when the underlying graph is a tree $T$, that is, a connected graph without cycles, where $2 \leq \operatorname{deg}(x)<\infty$ for every vertex $x$. There are several choices (one for each element in the boundary of the tree, see below) to equip the edge set of $T$ with an orientation such that each vertex $x$ has a unique predecessor $x^{-}$and $\operatorname{deg}(x)-1$ successors $y \in T$ such that $y^{-}=x$. Then it is easily understood that in the induced partial order, the ancestor relation $\preccurlyeq$, any two vertices $x, y \in T$ have a greatest common ancestor $x \curlywedge y$. If $o \in T$ is a reference vertex (origin), then we define $\mathfrak{h}(x)=d(x, o \curlywedge x)-d(o, o \curlywedge x)$, where $d(\cdot, \cdot)$ denotes the usual graph metric (cf. e.g., the seminal paper of Cartier [17]). Then $(T, \mathfrak{h})$ is the typical example of a Busemann pair.

In the present paper, we shall deal with homogeneous trees $T=\mathbb{T}_{q}$, where each vertex has degree $q+1(q \geq 2)$. In this case, the horocyclic structure (i.e., the ancestor relation) is unique up to isomorphism. We write $\operatorname{DL}\left(q_{1}, \ldots, q_{d}\right)$ for the horocyclic product of the trees $T_{1}=\mathbb{T}_{q_{1}}, \ldots, T_{d}=\mathbb{T}_{q_{d}}$. The "DL" stands for Diestel and Leader, who were the first [23] to introduce the graph $\operatorname{DL}(2,3)$ in an attempt to answer a question raised by Woess [44, 41]: "is there a locally finite vertex-transitive graph which is not quasiisometric with a Cayley graph of any finitely generated group?" A very recent paper by Eskin, Fisher and Whyte [25] confirms that the graphs $\operatorname{DL}\left(q_{1}, q_{2}\right)$ for $q_{1} \neq q_{2}$ are such examples. (Recall that a graph is called vertex-transitive if its isometry group acts transitively on the vertex set. Recall also that being quasi-isometric-see $\$ 3 \mathrm{~B}$ for the definition-is far more general than being isometric.)

The purpose of this paper is to present a picture of many interesting features of the graphs $\mathrm{DL}\left(q_{1}, \ldots, q_{d}\right)$.

In $\$ 2$, we first recall in more detail the horocyclic structure of the homogeneous tree $\mathbb{T}_{q}$ and the group Aff $\left(\mathbb{T}_{q}\right)=\operatorname{Aut}\left(\mathbb{T}_{q}, \mathfrak{h}\right)$ of all its Busemann self-isometries. This group has been called the affine group of the tree by analogy with the affine group over $\mathbb{R}$ acting on the hyperbolic upper half-plane. We determine the full isometry group Aut(DL) of $D L=$ $\operatorname{DL}\left(q_{1}, \ldots, q_{d}\right)$. We prove that it is a finite extension of the group $\mathcal{A}=\prod_{j=1}^{d} \mathfrak{h} \operatorname{Aff}\left(\mathbb{T}_{q_{j}}\right)$. The group $\mathcal{A}$ acts transitively on $\mathrm{DL}$ and is amenable as a locally compact, totally disconnected group with the topology of pointwise convergence.

If the $q_{j}$ do not all coincide, we show that the group $\mathcal{A}$ is also non-unimodular (i.e., the left Haar measure is not right-invariant). Consequently, by a theorem of Soardi and 
Woess [41], the graph DL is non-amenable, i.e., it satisfies a strong isoperimetric inequality (the Cheeger inequality). We also conclude that Aut(DL) cannot have a co-compact lattice, that is, there is no discrete (closed) subgroup that acts on DL with finitely many orbits. In particular, if the $q_{j}$ do not all coincide, then DL is vertex-transitive, but is not the Cayley graph of a finitely generated group.

In $\$ 3$, we study $\operatorname{DL}_{d}(q)=\operatorname{DL}(q, \ldots, q)$, the horocyclic product of $d$ copies of $\mathbb{T}_{q}$. We use an approach that is reminiscent of the method for constructing lattices in Lie groups over local fields, as outlined on the first page of the book by Margulis [35]. When $q=p_{1} \cdots p_{r}$ is the factorization of $q$ as a product of prime powers, and $p_{\iota} \geq d-1$ for all $\iota \in\{1, \ldots, r\}$, the graph $\operatorname{DL}_{d}(q)$ is a Cayley graph of a group of affine matrices over a ring of Laurent polynomials whose coefficients come from a suitable finite ring. There is some degree of freedom in the choice of the ring of coefficients. When $d=2$ or $d=3$, we can take the ring $\mathfrak{Z}_{q}=\mathbb{Z} / q \mathbb{Z}$ of integers modulo $q$, and for $d=2$ this is a way to describe the lamplighter group $\mathfrak{Z}_{q} 2 \mathbb{Z}$, while for $d=3$ we obtain a finitely presented group into which the lamplighter group embeds. This group has appeared in previous work by Baumslag [5] and others. The graph $\mathrm{DL}_{d}(q)$ is quasi-isometric with $\mathrm{DL}_{d}\left(q^{s}\right)$ for every $s \geq 1$, so $\mathrm{DL}_{d}(q)$ is always quasi-isometric with a Cayley graph of a finitely generated group. On the other hand, [23] and [25] suggest that the vertex-transitive graph $\mathrm{DL}\left(q_{1}, \ldots, q_{d}\right)$ is not quasi-isometric with any Cayley graph when the $q_{j}$ do not all coincide.

In $\$ 4$, we consider $\operatorname{DL}\left(q_{1}, \ldots, q_{d}\right)$ as a $(d-1)$-dimensional cell complex and explore its homotopy type, which is that of a union of countably many $(d-1)$-spheres glued together at a single point. This should be compared with a deep theorem of Bestvina and Brady [7]. Thus, when DL is a Cayley graph of a group, then this group is of type $F_{d-1}$, but not of type $F_{d}$, and in particular it is finitely presented when $d \geq 3$. We deduce that, for each $d$, the lamplighter group can be embedded in a metabelian group of type $F_{d}$. In general, it is known [10] that every metabelian group embeds in a metabelian group of type $F_{3}$, while embeddability in $F_{d}$ for larger $d$ is conjectured.

In $\$ 5$, we turn our attention to a more analytic-probabilistic object. The simple random walk on any locally finite, connected graph $X$ is the Markov chain whose transition matrix $P=(p(x, y))_{x, y \in X}$ is given by

$$
p(x, y)= \begin{cases}1 / \operatorname{deg}(x) & \text { if } y \sim x, \\ 0 & \text { otherwise. }\end{cases}
$$

$P$ acts on functions $f: X \rightarrow \mathbb{R}$ by

$$
P f(x)=\sum_{y} p(x, y) f(y) .
$$

In our case, $\operatorname{deg}(\cdot)=(d-1)\left(q_{1}+\cdots+q_{d}\right)$ is constant, and we are interested in the spectrum of $P$ on the space $\ell^{2}(\mathrm{DL})$ of all square-summable complex functions on $\mathrm{DL}$. The spectral radius $\rho(P)$ is equal to 1 if and only if $q_{1}=\cdots=q_{d}$. As a set, $\operatorname{spec}(P)$ is an interval contained in $[-1 /(d-1), \rho(P)]$ and, with the exception of a "degenerate" case, it coincides with it. In particular, for $\mathrm{DL}_{d}(q)$ the spectrum of $P$ is the same as the spectrum of $P$ 's projection to the lattice $\mathbb{A}_{d-1}$. This last spectrum is absolutely contin- 
uous. On the other hand, for arbitrary $q_{1}, \ldots, q_{d}$, the spectrum of $P$ on $\operatorname{DL}\left(q_{1}, \ldots, q_{d}\right)$ is pure point: there is an orthonormal basis of $\ell^{2}(\mathrm{DL})$ that consists of finitely supported eigenfunctions of $P$. This extends previous results regarding the lamplighter group and the basic Diestel-Leader graphs $\operatorname{DL}\left(q_{1}, q_{2}\right)$ (see Grigorchuk and Żuk [29], Dicks and Schick [22] and Bartholdi and Woess [3]). For the case $d=2$, the eigenvalues and eigenfunctions were computed explicitly in those references. Here, we present explicit computations for $d=3$ and $\mathrm{DL}_{3}(q)$, while the general case seems intractable (except numerically).

Finally, in $\$ 6$, we study the general class of random walks on DL whose transition matrix is irreducible, invariant under the group $\mathcal{A}$, and has finite first moment. Using results of Cartwright, Kaimanovich and Woess [19] and Brofferio [12] concerning random walks on $\operatorname{Aff}\left(\mathbb{T}_{q}\right)$, we show that those random walks on DL converge almost surely to the geometric boundary of DL. This boundary is the ideal boundary added to DL when considering the closure of DL in $\prod_{i} \widehat{T}_{i}$, where $\widehat{T}_{i}$ is the well-known end compactification of $T_{i}$. We then use the ray criterion of Kaimanovich (see [32]) to prove that the active part of the boundary (i.e., the support of the limit distribution of the random walk) is the "largest possible" model for distinguishing limit points of the random walk: it is the Poisson boundary.

\section{Isometry groups}

We start with a picture of the homogeneous tree $\mathbb{T}_{2}$ in horocyclic layers (Fig. 1), since it will be useful throughout the paper to keep this description in mind. Note that the negative direction is "upwards" in the picture.

Along with that picture comes a more detailed description of the geometry of $T=\mathbb{T}_{q}$.

Any pair of vertices $x$ and $y$ is connected by a unique geodesic path $\overline{x y}$ whose length (number of edges) is the distance $d(x, y)$. A geodesic ray is a one-sided infinite geodesic path (isometric embedding of a half-line graph). Two rays are called equivalent if their symmetric difference (as sets of vertices) is finite. An end of $T$ is an equivalence class of rays. The boundary $\partial T$ of $T$ is the set of ends of $T$. For each $\xi \in \partial T$ and each $x \in T$ there is a unique geodesic ray $\overline{x \xi}$ that represents $\xi$ and starts with $x$. We choose an origin (root) $o \in T$ and write $|x|=d(x, o)$. If $w, z \in \widehat{T}=T \cup \partial T$ then their confluent $c(w, z)$ is the last common element on $\overline{o w}$ and $\overline{o z}$, a vertex of $T$ unless $w=z \in \partial T$. With the ultrametric

$$
\theta(w, z)= \begin{cases}q^{-|c(w, z)|} & \text { if } w \neq z \\ 0 & \text { if } w=z\end{cases}
$$

$\widehat{T}$ becomes a compact space.

We now select an end $\omega \in \partial T$ and write $\partial^{*} T=\partial T \backslash\{\omega\}$. Given $\omega$, we can define the predecessor $x^{-}$of $x \in T$ as the neighbour of $T$ that lies on $\bar{x}$. Thus, the ancestor relation is

$$
x \preccurlyeq y \Leftrightarrow x \in \overline{y \omega},
$$

and for $x, y$ in general position, $x \curlywedge y$ is the greatest common ancestor. We write $\mathfrak{u}(x, y)=$ 


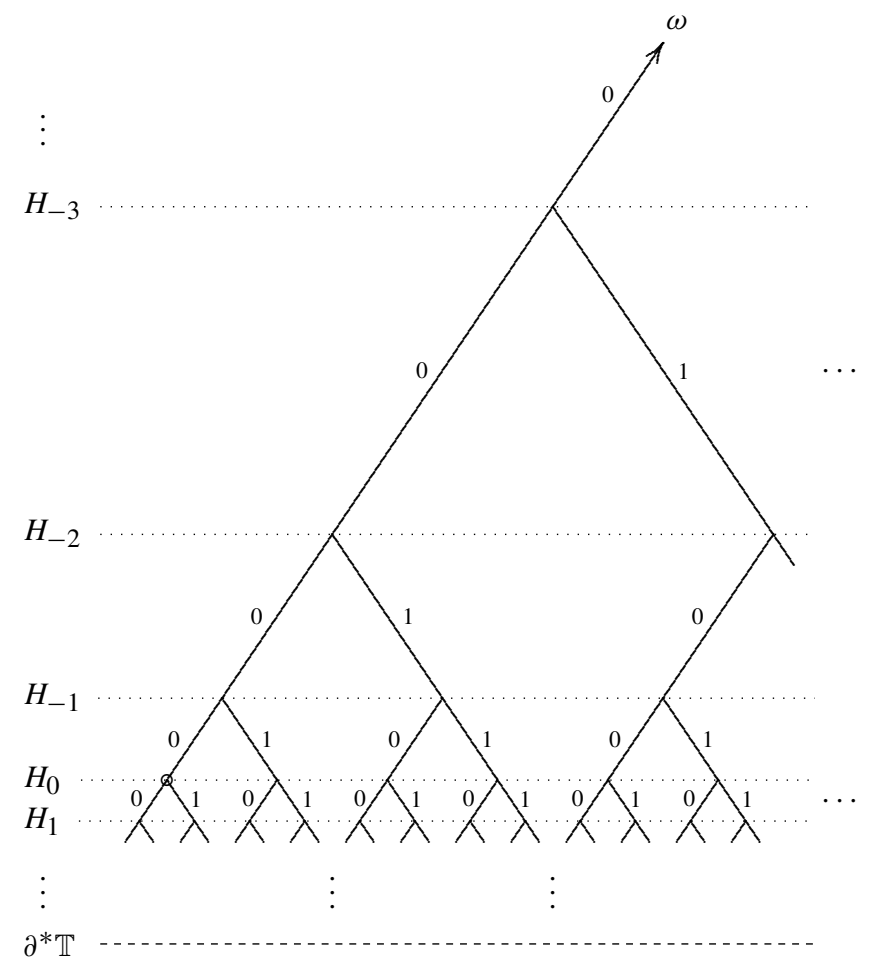

Fig. 1

$d(x, x \curlywedge y)$. Then the horocycle index of $x$ (the Busemann function with respect to $\omega$ ) is

$$
\mathfrak{h}(x)=\mathfrak{u}(x, o)-\mathfrak{u}(o, x),
$$

and the $k$-th horocycle is $H_{k}=\{x \in T: \mathfrak{h}(x)=k\}$. In particular,

$$
d(x, y)=\mathfrak{u}(x, y)+\mathfrak{u}(y, x) \text { and } \mathfrak{h}(x)-\mathfrak{h}(y)=\mathfrak{u}(x, y)-\mathfrak{u}(y, x) .
$$

As in Figure 1, we can label the edges of $T$ with the elements of $\mathfrak{Z}_{q}$ so that the edges between a vertex and its $q$ successors carry distinct labels, and so that on the geodesic from any vertex to $\omega$, only finitely many labels are non-zero. This labelling will be used several times in what follows.

For $T=\mathbb{T}_{q}$, its affine group Aff $\left(\mathbb{T}_{q}\right)$ is the stabilizer of $\omega$ in Aut $\left(\mathbb{T}_{q}\right)$. It is an amenable and non-unimodular closed subgroup of Aut $\left(\mathbb{T}_{q}\right)$ that acts transitively on $\mathbb{T}_{q}$, and all its elements are Busemann isometries. We have $\mathfrak{h}(g)=\mathfrak{h}(g o)$ for $g \in \operatorname{Aff}\left(\mathbb{T}_{q}\right)$. See Cartwright, Kaimanovich and Woess [19] for more details about the structure of Aff $\left(\mathbb{T}_{q}\right)$.

We shall need some basic facts about the modular function of an isometry group of a locally finite graph $X$ which is closed with respect to pointwise convergence. For more details, see Trofimov [43] and Woess [44]. If $\Gamma \leq \operatorname{Aut}(X)$ is such a group, and $x \in X$, 
then $\Gamma_{x}$ denotes the stabilizer of $x$ in $\Gamma$, while $\Gamma x$ is the orbit of $x$ under $\Gamma$. Since $\Gamma$ is locally compact, it carries a left Haar measure $d g$. The modular function $\Delta$ on $\Gamma$ is the unique multiplicative homomorphism $\Gamma \rightarrow \mathbb{R}_{+}$which satisfies

$$
\Delta\left(g_{0}\right) \int_{\Gamma} f\left(g g_{0}\right) d g=\int_{\Gamma} f(g) d g
$$

for every $g_{0} \in \Gamma$ and every continuous, compactly supported function $f$ on $\Gamma$. Inserting for $f$ the indicator function of $\Gamma_{x}$ (which is an open, compact subgroup of $\Gamma$ ), one finds the formula

$$
\Delta(g)=\left|\Gamma_{x}(g x)\right| /\left|\Gamma_{g x} x\right|
$$

for $g \in \Gamma$ and for arbitrary $x \in X$, where $\left|\Gamma_{x} y\right|$ is the (finite) number of elements in the $\Gamma_{x}$-orbit of $y$; see e.g. [43, 44]. In particular, one has the following.

(2.4) Lemma. If $\Gamma$ acts transitively on $X$ then $\Gamma$ is unimodular if and only if $\left|\Gamma_{x} y\right|=$ $\left|\Gamma_{y} x\right|$ for some ( $\Leftrightarrow$ every) $x \in X$ and all its neighbours $y$.

In the following, we fix integers $q_{1}, \ldots, q_{d} \geq 2$, and write $o_{j}$ for the origin of $T_{j}=\mathbb{T}_{q_{j}}$, while the symbol $o$ will be used for the origin $o=o_{1} \cdots o_{d}$ of $\mathrm{DL}=\mathrm{DL}\left(q_{1}, \ldots, q_{d}\right)$. If $x, y \in \mathrm{DL}$, then we say that a neighbour $y$ of $x$ has type $\mathbf{e}_{i}-\mathbf{e}_{j}$ if $y_{i}^{-}=x_{i}$ and $y_{j}=x_{j}^{-}$. In this case, $x$ is a neighbour with type $\mathbf{e}_{j}-\mathbf{e}_{i}$ of $y$. We write $N_{i, j}(x)$ for the set of neighbours with type $\mathbf{e}_{i}-\mathbf{e}_{j}$ of $x$.

(2.5) Proposition. The group

$$
\mathcal{A}=\mathcal{A}\left(q_{1}, \ldots, q_{d}\right)=\prod_{j=1}^{d} \mathfrak{h} \operatorname{Aff}\left(\mathbb{T}_{q_{j}}\right)
$$

acts transitively on $\mathrm{DL}=\mathrm{DL}\left(q_{1}, \ldots, q_{d}\right)$ via (1.4). It is amenable. Furthermore, $\mathcal{A}$ is unimodular if and only if $q_{1}=\cdots=q_{d}$.

Proof. Let $x=x_{1} \cdots x_{d}$ be in DL. Then there are $g_{j} \in \operatorname{Aff}\left(\mathbb{T}_{q_{j}}\right)$ such that $g_{j} o_{j}=x_{j}$, $j=1, \ldots, d$. Setting $g=g_{1} \cdots g_{d}$ as in 1.4 , we get $g \in \mathcal{A}$, since $\sum_{j} \mathfrak{h}\left(g_{j}\right)=$ $\sum_{j} \mathfrak{h}\left(x_{j}\right)=0$. Thus, $g o=x$, and the action is transitive. Amenability of $\mathcal{A}$ follows from the fact that it is a closed subgroup of the direct product of the locally compact amenable groups $\operatorname{Aff}\left(\mathbb{T}_{q_{j}}\right)$.

Regarding unimodularity, let $x$ be in DL. By construction, $\mathcal{A}_{x}$ must map every neighbour $y$ of $x$ of type $\mathbf{e}_{j}-\mathbf{e}_{i}$ to a neighbour of $x$ of the same type, and every permutation of this kind can be achieved. Now $x$ has exactly $q_{j}$ neighbours of type $\mathbf{e}_{j}-\mathbf{e}_{i}$. Therefore, $\left|\mathcal{A}_{x} y\right|=q_{j}$, and (by exchanging $x \leftrightarrow y$ and $\left.i \leftrightarrow j\right)\left|\mathcal{A}_{y} x\right|=q_{i}$. If we vary $i, j(i \neq j)$ and apply Lemma 2.4 , then we see that our group is unimodular if and only if all $q_{j}$ coincide.

Besides the elements of $\mathcal{A}$, there may be further isometries of DL. Let $\mathfrak{S}=\mathfrak{S}\left(q_{1}, \ldots, q_{d}\right)$ be the group of all permutations $\sigma$ of $\{1, \ldots, d\}$ such that $q_{\sigma(j)}=q_{j}$ for all $j$. Then $\mathfrak{S}$ 
acts on DL by

$$
\sigma x=x_{\sigma^{-1}(1)} \cdots x_{\sigma^{-1}(d)},
$$

that is, $\sigma$ permutes identical trees in the horocyclic product. Thus, $\mathfrak{S}$ also acts on $\mathcal{A}$ by group automorphisms $(\sigma, g) \mapsto g^{\sigma}=\sigma g \sigma^{-1}$. If all $q_{j}$ are distinct, then $\mathfrak{S}\left(q_{1}, \ldots, q_{d}\right)$ is of course trivial. We shall prove the following.

(2.7) Theorem. The full isometry group of $\mathrm{DL}\left(q_{1}, \ldots, q_{d}\right)$ is the semidirect product of $\mathfrak{S}$ with $\mathcal{A}$ with respect to the action $(\sigma, g) \mapsto g^{\sigma}$,

$$
\operatorname{Aut}(\mathrm{DL})=\mathfrak{S} \ltimes \mathcal{A} .
$$

Thus, Aut(DL) is amenable, and it is unimodular if and only if all $q_{i}$ coincide.

For the proof, we need a description of the (graph-theoretical) link $N(x)$ of a vertex $x \in \mathrm{DL}$, that is, the subgraph of DL spanned by the neighbours of $x$. Under the graph homomorphism $\mathfrak{H}: \mathrm{DL} \rightarrow \mathbb{A}_{d-1}$, where $\mathfrak{H}(x)=\mathbf{k}=\left(\mathfrak{h}\left(x_{1}\right), \ldots, \mathfrak{h}\left(x_{d}\right)\right)$, the link $N(x)$ maps onto the link $N(\mathbf{k})$ in the lattice $\mathbb{A}_{d-1}$. This last link has $(d-1)$-cliques (complete graphs on $d-1$ vertices) as its building blocks. Namely, for $i \in\{1, \ldots, d\}$, write

$$
S_{i}^{+}(\mathbf{k})=\left\{\mathbf{k}+\mathbf{e}_{i}-\mathbf{e}_{j}: j \neq i\right\} \quad \text { and } \quad S_{i}^{-}(\mathbf{k})=\left\{\mathbf{k}+\mathbf{e}_{j}-\mathbf{e}_{i}: j \neq i\right\} .
$$

Each of those spans a complete subgraph of $N(\mathbf{k})$. We have $N(\mathbf{k})=\bigcup_{i=1}^{d} S_{i}^{+}(\mathbf{k})=$ $\bigcup_{i=1}^{d} S_{i}^{-}(\mathbf{k})$,

$S_{i}^{+}(\mathbf{k}) \cap S_{j}^{+}(\mathbf{k})=S_{i}^{-}(\mathbf{k}) \cap S_{j}^{-}(\mathbf{k})=\emptyset \quad$ and $\quad S_{i}^{+}(\mathbf{k}) \cap S_{j}^{-}(\mathbf{k})=\left\{\mathbf{k}+\mathbf{e}_{i}-\mathbf{e}_{j}\right\} \quad(i \neq j)$.

We write $S_{i}^{ \pm}(x)$ for the set of all points in $N(x)$ which are mapped to $S_{i}^{ \pm}(\mathbf{k})$ by $\mathfrak{H}$. Note that $S_{i}^{+}(x) \cap S_{j}^{-}(x)=N_{i, j}(x)$. The edges in $N(x)$ are as follows.

(1) If $y, z \in S_{i}^{+}(x)$ then there are $j, k \neq i$ such that $\mathfrak{H}(y)=\mathbf{k}+\mathbf{e}_{i}-\mathbf{e}_{j}$ and $\mathfrak{H}(z)=\mathbf{k}+\mathbf{e}_{i}-\mathbf{e}_{k}$. In this case, there is an edge between $y$ and $z$ if and only if $j \neq k$ and $y_{i}=z_{i}$, in which case $z$ is a neighbour of type $\mathbf{e}_{j}-\mathbf{e}_{k}$ of $y$ (i.e., $z_{j}^{-}=y_{j}$ and $y_{k}^{-}=z_{k}$ ). Thus, the subgraph of $N(x)$ that is mapped onto an edge in $S_{i}^{+}(\mathbf{k})$ is the graph $D\left(q_{i}, q_{i}\right)$ consisting of $q_{i}$ independent edges with their endpoints; see Figure 2a.

(2) If $y, z \in S_{i}^{-}(x)$ then there are $j, k \neq i$ such that $\mathfrak{H}(y)=\mathbf{k}+\mathbf{e}_{j}-\mathbf{e}_{i}$ and $\mathfrak{H}(z)=\mathbf{k}+\mathbf{e}_{k}-\mathbf{e}_{i}$. In this situation, there is an edge between $y$ and $z$ if and only if $j \neq k$; furthermore, $z$ is a neighbour of type $\mathbf{e}_{k}-\mathbf{e}_{j}$ of $y$ (i.e., $z_{k}^{-}=y_{k}$ and $y_{j}^{-}=z_{j}$ ). Thus, the subgraph of $N(x)$ that is mapped onto the edge $\left[\mathbf{k}+\mathbf{e}_{j}-\mathbf{e}_{i}, \mathbf{k}+\mathbf{e}_{k}-\mathbf{e}_{i}\right]$ in $S_{i}^{+}(\mathbf{k})$ is the complete bipartite graph $K\left(q_{j}, q_{k}\right)$; see Figure $2 \mathrm{~b}$.

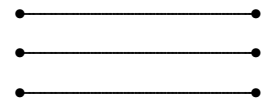

Fig. 2a. $D(3,3)$

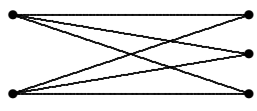

Fig. 2b. $K(2,3)$ 


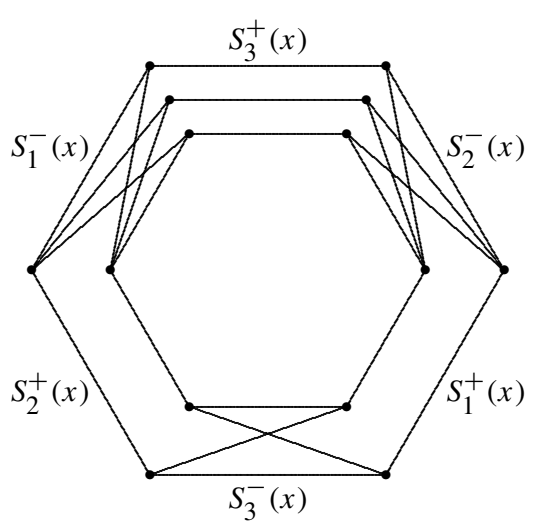

Fig. 3a. $N(x)$ in $\operatorname{DL}(2,2,3)$

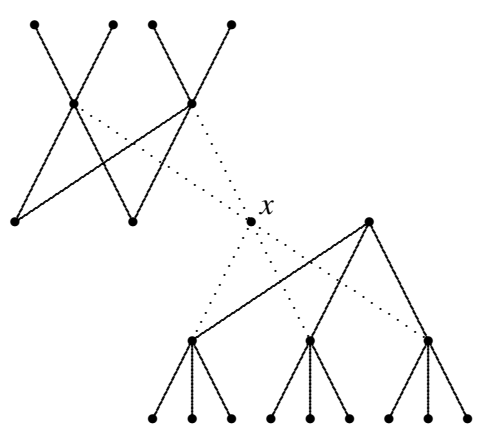

Fig. 3b. $N_{2}(x)$ in $\operatorname{DL}(3,2)$

Figure 3a shows the link of a vertex of $\mathrm{DL}(2,2,3)$. When $d \geq 3$, the link is connected. When $d=2$, it consists of $q_{1}+q_{2}$ isolated points, and in this case, it will be more useful to consider the 2-link $N_{2}(x)$ spanned by all points at distance 1 and 2 from $x$. Each of the $q_{1}$ neighbours $v$ of type $\mathbf{e}_{1}-\mathbf{e}_{2}$ of $x=x_{1} x_{2}$ is connected by an edge to each of the $q_{2}-1$ points $x_{1} y_{2}$, where $y_{2} \neq x_{2}$ is a sibling of $x_{2}$ in $T_{2}$, that is, $y_{2}^{-}=x_{2}^{-}$. In turn, there is an edge between each of those neighbours $v$ of $x$ and its $q_{1}$ neighbours of the same type $\mathbf{e}_{1}-\mathbf{e}_{2}$. Exchanging the role of $\mathbf{e}_{1}$ and $\mathbf{e}_{2}$, one finds the other part of $N_{2}(x)$. See Figure $3 \mathrm{~b}$, where we have also drawn the edges from $x$ to its neighbours in dotted lines.

(2.8) Lemma. For every $x \in \mathrm{DL}$ and every $g$ in the stabilizer Aut(DL) $)_{x}$ of $x$, there is $\sigma \in \mathfrak{S}$ such that for all $i, j(i \neq j)$, we have $g N_{i, j}(x)=N_{\sigma^{-1}(i), \sigma^{-1}(j)}(x)$.

Proof. Our $g$ acts as a graph isometry on $N(x)$, and also on $N_{2}(x)$. If $d=2$ (see Figure $3 b$ ) then $g$ must permute the two connected components of $N_{2}(x)$. This permutation must be trivial unless $q_{1}=q_{2}$. Thus, the statement follows when $d=2$.

If $d \geq 3$, then by the above, each $S_{i}^{-}(x)$ is a complete $(d-1)$-partite subgraph of $N(x)$. That is, its vertex set is partitioned into the sets $N_{j, i}(x)$ having cardinality $q_{j}$, $j \neq i$, such that every pair of vertices in $N_{j, i}(x) \times N_{k, i}(x), k \neq j$, is connected by an edge, while there are no edges between different vertices within each $N_{j, i}(x)$. Also, $S_{i}^{-}(x)$ is a maximal complete $(d-1)$-partite subgraph of $N(x)$ (i.e., it is not contained in any bigger complete $(d-1)$-partite subgraph). This property must be preserved by isometries of $N(x)$, and $g S_{i}^{-}(x)$ must again be a maximal complete $(d-1)$-partite subgraph of $N(x)$, whose $d-1$ classes must have the same cardinalities $q_{j}$ as the sets $N_{i, j}(x), j \neq i$. Thus, there is a permutation $\sigma$ of $\{1, \ldots, d\}$ such that $g S_{i}^{-}(x)=S_{\sigma^{-1}(i)}^{-}(x)$, and from $\left|\left\{j \neq i: q_{j}=q_{i}\right\}\right|=\left|\left\{k \neq \sigma^{-1}(i): q_{k}=q_{i}\right\}\right|$ we deduce $q_{i}=q_{\sigma^{-1}(i)}$ for each $i$. Therefore, $\sigma \in \mathfrak{S}$.

For each $j \neq i$, we must have $g N_{j, i}(x)=N_{k, \sigma^{-1}(i)}(x)$ and $g N_{i, j}(x)=N_{l, \sigma^{-1}(j)}(x)$ for some $k \neq \sigma^{-1}(i), l \neq \sigma^{-1}(j)$. We still have to show that $k=\sigma^{-1}(j)$, and consequently $l=\sigma^{-1}(i)$. Now note that with respect to its ("inner") graph metric, $N(x)$ has diameter 3 , and that the only points at distance 3 from all $y \in N_{i, j}(x)$ are precisely those 
in $N_{j, i}(x)$. Therefore

$$
N_{l, \sigma^{-1}(j)}(x)=g N_{i, j}(x)=N_{\sigma^{-1}(i), k}(x)
$$

as sets, and thus $\left(l, \sigma^{-1}(j)\right)=\left(\sigma^{-1}(i), k\right)$. This completes the proof of the lemma.

We shall also need the following preparatory lemma.

(2.9) Lemma. If $g \in \operatorname{Aut}(\mathrm{DL})$ satisfies $g N_{i, j}(x)=N_{i, j}(g x)$ for all $i, j(i \neq j)$, then there is $h \in \mathcal{A}$ such that $\left.\left.g\right|_{N(x)} \equiv h\right|_{N(x)}$.

(As a matter of fact, it will turn out below that $g$ itself must belong to $\mathcal{A}$.)

Proof. For each pair $(i, j)$, the isometry $g$ maps the $q_{i}$ elements of $N_{i, j}(x)$ to those of $N_{i, j}(g x)$. By the structure of $S_{i}^{+}(x)$ and $S_{i}^{+}(g x)$, this map must be independent of $j$ $(j \neq i)$. That is, there is a map $h_{i}$ between the successors of $x_{i}$ in $T_{i}$ and those of $(g x)_{i}$ such that $(g y)_{i}=h_{i} y_{i}$ for all $y \in N_{i, j}(x)$ and all $j \neq i$. This permutation can be extended to an isometry of $T_{i}$, again denoted $h_{i}$, that maps $x_{i}$ to $(g x)_{i}$ and maps the branches of $T_{i}$ "below" $x_{i}$ to those "below" $(g x)_{i}$ according to the map of $y_{i}$ to $(g y)_{i}$. Setting $h=h_{1} \cdots h_{d}$ according to [1.4], we obtain the required element of $\mathcal{A}$.

Proof of Theorem 2.7. Consider an arbitrary $g \in \operatorname{Aut(DL).~Set~} x=g^{-1} o$, where $o$ is the root of DL. Then there is $g_{x} \in \mathcal{A}$ such that $x=g_{x}^{-1} o$, whence $g g_{x}^{-1} o=o$. By Lemma 2.8 there is $\sigma \in \mathfrak{S}$, acting on DL by 2.6, such that $g^{\prime}=\sigma^{-1} g g_{x}^{-1} \in \operatorname{Aut}(\mathrm{DL})_{o}$ satisfies $g^{\prime} N_{i, j}(o)=N_{i, j}(o)$ for all $i, j$. We claim that $g^{\prime}$ is type-preserving, that is, for all $x \in \mathrm{DL}$,

$$
g^{\prime} N_{i, j}(x)=N_{i, j}\left(g^{\prime} x\right) \quad \forall i, j \in\{1, \ldots, d\}(i \neq j) .
$$

(Note that this does hold for every $g^{\prime} \in \mathcal{A}$.) Since DL is connected and 2.10 is true for $x=o$, it is sufficient to show the following.

$$
\text { If 2.10) holds for some } x \in \mathrm{DL} \text { then it holds for every } y \in N(x) \text {. }
$$

So suppose that 2.10$)$ holds for some $x$. Let $h \in \mathcal{A}$ be as in Lemma 2.9, associated with $g^{\prime}$, and set $g^{\prime \prime}=h^{-1} g^{\prime}$. Then $g^{\prime \prime} v=v$ for every $v \in\{x\} \cup N(x)$. Let $y \in N(x)$, so that $y \in N_{i, j}(x)$ for some $i, j$. Since $g^{\prime \prime} y=y$, Lemma 2.8 implies that there is $\tau \in \mathfrak{S}$ such that $g^{\prime \prime} N_{i, j}(y)=N_{\tau^{-1}(i), \tau^{-1}(j)}(y)$ for all $i, j$.

Since $x \in N_{j, i}(y)$ and $g^{\prime \prime} x=x$, we find that $\tau(i)=i$ and $\tau(j)=j$. Also, if $k \neq i, j$, then $N_{k, i}(y)=N_{k, j}(x)$ is stabilized by $g^{\prime \prime}$. Thus, $\tau(k)=k$. We see that $\tau$ is the identity, and as $h \in \mathcal{A}$,

$$
g^{\prime} N_{i, j}(y)=h g^{\prime \prime} N_{i, j}(y)=h N_{i, j}(y)=N_{i, j}(h y)=N_{i, j}\left(g^{\prime} y\right),
$$

as claimed. This proves 2.11) and consequently 2.10).

We now use 2.10) to show that $g^{\prime} \in \mathcal{A}$. Our claim is the following.

(2.12) If $x, y \in \mathrm{DL}$ satisfy $x_{i}=y_{i}$ for some $i \in\{1, \ldots, d\}$ then $\left(g^{\prime} x\right)_{i}=\left(g^{\prime} y\right)_{i}$. 
Indeed, if this holds, then define $g_{i} \in \operatorname{Aff}\left(\mathbb{T}_{q_{i}}\right)$ as follows. Given $x_{i} \in T_{i}$, choose $x \in \mathrm{DL}$ with $i$-th coordinate $x_{i}$, and set $g_{i} x_{i}=\left(g^{\prime} x\right)_{i}$. This is independent of the specific choice of $x$ by 2.12). We therefore get $g^{\prime}=g_{1} \cdots g_{d} \in \mathcal{A}$.

For any $d$ and $i, j \in\{1, \ldots, d\}$ with $i \neq j$, we can define recursively $N_{i, j}^{(0)}(x)=\{x\}$ and $N_{i, j}^{(k)}(x)=\bigcup\left\{N_{i, j}(y): y \in N_{i, j}^{(k-1)}(x)\right\}$, where $x \in \mathrm{DL}$. We observe that

$$
v_{j}=w_{j} \quad \text { for all } v, w \in N_{i, j}^{(k)}(x) .
$$

The proof of 2.12 is different in the cases $d=2$ and $d \geq 3$. In both cases, we may assume without loss of generality that $i=1$ in 2.12). Suppose therefore that $x, y \in \mathrm{DL}$ satisfy $x_{1}=y_{1}$.

Case 1: $d=2$. Consider $x_{2}, y_{2}$ and their common ancestor $u_{2}=x_{2} \curlywedge y_{2}$ in $T_{2}$. Since $x_{1}=y_{1}$, we have $\mathfrak{h}\left(x_{2}\right)=\mathfrak{h}\left(y_{2}\right)$, whence $d\left(x_{2}, u_{2}\right)=d\left(y_{2}, u_{2}\right)=\mathfrak{h}\left(x_{2}\right)-\mathfrak{h}\left(u_{2}\right)=$ : $k \geq 0$. We can find $u_{1} \in T_{1}$ such that $x_{1} \preccurlyeq u_{1}$ and $\mathfrak{h}\left(x_{1}\right)-\mathfrak{h}\left(u_{1}\right)=-k$, so that $u=u_{1} u_{2} \in \mathrm{DL}$ and $x, y \in N_{2,1}^{(k)}(u)$. Since $g^{\prime}$ is type-preserving, we have

$$
g^{\prime} N_{2,1}^{(k)}(u)=N_{2,1}^{(k)}\left(g^{\prime} u\right) \ni g^{\prime} x, g^{\prime} y
$$

Using the above observation, we get $\left(g^{\prime} x\right)_{1}=\left(g^{\prime} y\right)_{1}$, which proves 2.12).

Case 2: $d \geq 3$. The subgraph of $\operatorname{DL}\left(q_{1}, \ldots, q_{d}\right)$ spanned by the set $\left\{v=v_{1} \cdots v_{d} \in \mathrm{DL}\right.$ : $\left.v_{1}=x_{1}\right\}$ is connected; indeed, it is isomorphic to $\operatorname{DL}\left(q_{2}, \ldots, q_{d}\right)$. Thus, there is a path from $x$ to $y$ in $\mathrm{DL}$ all of whose vertices have the same first coordinate $x_{1}$ : if $v, w$ are successive vertices on this path then $w \in N_{i, j}(v)$ where $i, j \neq 1$, and $v_{1}=w_{1}=x_{1}$. But then 2.10 implies $g^{\prime} w \in N_{i, j}\left(g^{\prime} v\right)$, so that $g^{\prime} v$ and $g^{\prime} w$ differ only in the $i$-th and $j$-th coordinates. In particular, $\left(g^{\prime} w\right)_{1}=\left(g^{\prime} v\right)_{1}$, whence inductively $\left(g^{\prime} y\right)_{1}=\left(g^{\prime} x\right)_{1}$.

We conclude that $g=\sigma g^{\prime}$, where $\sigma \in \mathfrak{S}$ and $g^{\prime} \in \mathcal{A}$, so that we have completed the description of Aut(DL).

If $h \in \mathcal{A}$ and $g=\sigma g^{\prime} \in \operatorname{Aut}(\mathrm{DL})$ with $\sigma \in \mathfrak{S}$ and $g^{\prime} \in \mathcal{A}$, then $g h g^{-1}=$ $\sigma\left(g^{\prime} h g^{\prime-1}\right) \sigma^{-1}$ is type-preserving, so that 2.12 implies $g h g^{-1} \in \mathcal{A}$. It is now obvious that the factor group $\operatorname{Aut}(\mathrm{DL}) / \mathcal{A}$ is $\mathfrak{S}$.

We briefly remind the reader of the concept of amenability of a locally compact group (三 existence of a finitely additive, left invariant probability measure on the group); see Paterson [37]. Recall that a locally finite graph $X$ is called amenable if its isoperimetric constant is 0 , that is, the number

$$
\kappa=\inf \{|\partial F| / \operatorname{Vol}(F): F \subset X \text { finite }\}
$$

where $|\cdot|$ is cardinality, $\partial F$ is the set of edges between $F$ and $X \backslash F$, and $\operatorname{Vol}(F)=$ $\sum_{x \in F} \operatorname{deg}(x)$. By the well-known criterion of Følner, a finitely generated group is amenable if and only if one ( $\Leftrightarrow$ each) of its Cayley graphs is amenable in the latter sense.

(2.13) Corollary. The graph $\operatorname{DL}\left(q_{1}, \ldots, q_{d}\right)$ is amenable if and only if all $q_{i}$ coincide. 
Proof. By a theorem of Soardi and Woess [41], a vertex-transitive graph is amenable if and only if its automorphism group (or equivalently, any closed transitive subgroup) is both amenable and unimodular. Theorem 2.7 yields the result.

A co-compact lattice in a locally compact group is a discrete, countable subgroup such that the factor space is compact. The following is standard.

(2.14) Lemma. If $X$ is a locally finite, vertex-transitive graph, then a subgroup $\Gamma$ of Aut $(X)$ is a co-compact lattice if and only if $\Gamma$ acts on $X$ with finitely many orbits and finite vertex stabilizers.

Proof. We prove the "only if" part; the "if" part is then an easy exercise. If $\Gamma$ is cocompact then there is a relatively compact set $K \subset \operatorname{Aut}(X) \operatorname{such}$ that $\operatorname{Aut}(X)=\Gamma K$. If $o \in X$ is arbitrarily chosen (a "root"), then relative compactness of $K$ means that $F=K o \subset X$ is finite. Transitivity of $\operatorname{Aut}(X)$ implies that $\Gamma F=X$, whence $\Gamma$ acts with finitely many orbits.

Suppose that for some $x$ the stabilizer $\Gamma_{x}$ contains a sequence of distinct elements. Since the stabilizer of $x$ in $\operatorname{Aut}(X)$ is compact, that sequence must have an accumulation point. This contradicts discreteness of $\Gamma$.

A non-unimodular group cannot contain a co-compact lattice. Recall that when $\Gamma$ is a finitely generated group and $S=S^{-1} \subset \Gamma$ is a finite set of generators, then the Cayley graph $X(\Gamma, S)$ of $\Gamma$ with respect to $S$ has vertex set $\Gamma$, and the edges are all $[x, y]$, where $x, y \in \Gamma$ and $x^{-1} y \in S$. The group $\Gamma$ acts by left multiplication on $X(\Gamma, S)$, the action is transitive, and all vertex stabilizers are trivial. In view of Theorem 2.7, we now get the following.

(2.15) Corollary. Assume that the $q_{j}$ do not all coincide; then Aut(DL) does not possess any co-compact lattice. Therefore, $\mathrm{DL}\left(q_{1}, \ldots, q_{d}\right)$ cannot be a Cayley graph of a finitely generated group.

\section{Cayley graphs}

We now study in more detail the case when $q_{1}=\cdots=q_{d}=q$ all coincide. In this case, we write $\mathrm{DL}_{d}(q)$ for the horocyclic product of $d$ trees with degree $q+1$. The fact that $\mathrm{DL}_{2}(q)=\operatorname{DL}(q, q)$ is a Cayley graph of the lamplighter group $\mathfrak{Z}_{q} 2 \mathbb{Z}$, where $\mathfrak{Z}_{q}=$ $\mathbb{Z} / q \mathbb{Z}$, has been explained (and exploited) in the papers by Woess [46] alone and with Bartholdi [3] and Brofferio [14]. We now study the general case. For investigations in a similar spirit, but with different methods and applied to different graphs (one-skeletons of affine buildings), see e.g. Cartwright and Steger [20].

Let $\mathfrak{L}_{q}$ be a commutative ring of order $\left|\mathfrak{L}_{q}\right|=q$ with multiplicative unit 1 , and choose $\ell \in \mathfrak{L}_{q}$. Consider the ring $\mathfrak{L}_{q}((\mathrm{t}+\ell))$ of all formal Laurent series

(3.1) $\mathrm{f}=\mathrm{f}(\mathrm{t}+\ell)=\sum_{k=-\infty}^{\infty} a_{k}(\mathrm{t}+\ell)^{k}, \quad a_{k} \in \mathfrak{L}_{q}, \quad \exists n_{0} \in \mathbb{Z}: a_{k}=0 \forall k<n_{0}$. 
If $a_{n_{0}} \neq 0$ in this representation, then we write $v_{\ell}(\mathrm{f})=n_{0}$ for the valuation of $\mathrm{f}$ with respect to $t+\ell$, setting $v_{\ell}(0)=\infty$. The corresponding absolute value is

$$
|\mathrm{f}|_{\ell}=q^{-\mathrm{v}_{\ell}(\mathrm{f})}
$$

It defines a complete ultrametric on $\mathfrak{L}_{q}((\mathrm{t}+\ell))$. Every closed ball in this ultrametric has radius $q^{n}$ for some $n \in \mathbb{Z}$ and is also open.

We shall write $\mathfrak{L}_{q}((\mathrm{t}+\ell))^{*}$ for the multiplicative group of invertible elements in $\mathfrak{L}_{q}((\mathrm{t}+\ell))$.

Following the explanation given in [19], we can identify $\mathfrak{L}_{q}((\mathrm{t}+\ell))$ with the "lower" boundary $\partial^{*} \mathbb{T}_{q}$, where the vertex set of $\mathbb{T}_{q}$ is the set of all closed balls $x=B_{\ell}\left(\mathrm{f}, q^{n}\right)$ with radius $q^{n}, n \in \mathbb{Z}$, and centre $\mathrm{f} \in \mathfrak{L}_{q}((\mathrm{t}+\ell))$. Relating this to the description of $\S 2$, the predecessor of vertex $x$ as above is $x^{-}=B_{\ell}\left(\mathrm{f}, q^{n+1}\right)$. The vertices on the $n$-th horocycle are, by definition, precisely all balls with radius $q^{-n}$, which form a partition of $\mathfrak{L}_{q}((\mathrm{t}+\ell))$.

Conversely, starting with $\mathbb{T}_{q}$, for any $k \in \mathbb{Z}$, we can label the edges between each vertex in $H_{k}$ and its $q$ successors with the elements of $\mathfrak{L}_{q}$ as in Figure 1, so that the sequence of labels on the geodesic from $\omega$ to $x \in H_{n}$ is $\left(a_{k}\right)_{k<n}$ with only finitely many $a_{k}$ non-zero. Then $x=B_{\ell}\left(\mathrm{g}, q^{-n}\right)$, where $\mathrm{g}(\mathrm{t})=\sum_{k<n} a_{k}(\mathrm{t}+\ell)^{k}$.

Using this description, one sees that the group $\operatorname{Aff}\left(\mathfrak{L}_{q}((\mathrm{t}+\ell))\right)$ of all affine matrices

$$
g=\left(\begin{array}{ll}
\mathrm{a} & \mathrm{b} \\
0 & 1
\end{array}\right), \quad \mathrm{a} \in \mathfrak{L}_{q}((\mathrm{t}+\ell))^{*}, \mathrm{~b} \in \mathfrak{L}_{q}((\mathrm{t}+\ell))
$$

embeds into $\operatorname{Aff}\left(\mathbb{T}_{q}\right)$ as a closed subgroup that acts transitively on the tree. Namely, the image of a ball $x=B_{\ell}\left(\mathrm{f}, q^{n}\right)$ as above under such a matrix is the ball

$$
g x=B_{\ell}\left(\mathrm{b}+\mathrm{a} \cdot \mathrm{f}, q^{n-m}\right), \quad \text { where } \quad m=\mathrm{v}_{\ell}(\mathrm{a}) .
$$

In the same way, we can also consider the ring $\mathfrak{L}_{q}\left(\left(\mathrm{t}^{-1}\right)\right)$ of all formal Laurent series $\mathrm{f}=\sum_{k} a_{k} \mathrm{t}^{-k}$ over $\mathfrak{L}_{q}$ in the variable $\mathrm{t}^{-1}$ with the valuation $\mathrm{v}_{\infty}(\mathrm{f})=n_{0}$ if $n_{0}$ is minimal such that $a_{n_{0}} \neq 0$. Again, we have the identification with $\partial^{*} \mathbb{T}_{q}$, but in this specific case, we define the $n$-th horocycle to consist of all closed balls in $\mathfrak{L}_{q}\left(\left(\mathrm{t}^{-1}\right)\right)$ with radius $q^{-n-1}$. Again, the group $\operatorname{Aff}\left(\mathfrak{L}_{q}\left(\left(\mathrm{t}^{-1}\right)\right)\right)$ embeds into $\operatorname{Aff}\left(\mathbb{T}_{q}\right)$ as a closed subgroup that acts transitively.

Now suppose that there are distinct elements $\ell_{1}, \ldots, \ell_{d-1} \in \mathfrak{L}_{q}$ such that their pairwise differences $\ell_{i}-\ell_{j}(i \neq j)$ are invertible. Consider the ring

$$
\mathcal{R}_{d}\left(\mathfrak{L}_{q}\right)=\mathfrak{L}_{q}\left[\left(\mathrm{t}+\ell_{1}\right)^{-1}, \ldots,\left(\mathrm{t}+\ell_{d-1}\right)^{-1}, \mathrm{t}\right]
$$

of all polynomials over $\mathfrak{L}_{q}$ in the variables $\left(\mathrm{t}+\ell_{1}\right)^{-1}, \ldots,\left(\mathrm{t}+\ell_{d-1}\right)^{-1}$, $\mathrm{t}$. They are of the form

$$
\mathrm{P}=\mathrm{P}\left(\mathrm{t}+\ell_{1}, \ldots, \mathrm{t}+\ell_{d-1}\right)=\sum_{k_{1}, \ldots, k_{d-1} \in \mathbb{Z}} a_{k_{1}, \ldots, k_{d-1}}\left(\mathrm{t}+\ell_{1}\right)^{k_{1}} \cdots\left(\mathrm{t}+\ell_{d-1}\right)^{k_{d-1}},
$$

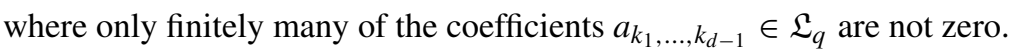

Note that $\mathcal{R}_{d}\left(\mathfrak{L}_{q}\right)$ depends not only on $d$ and $\mathfrak{L}_{q}$, but also on the choice of the $\ell_{i}$. The same is true for the group $\Gamma_{d}\left(\mathfrak{L}_{q}\right)$ in the next theorem. 
(3.6) Theorem. If $\mathfrak{L}_{q}$ contains distinct elements $\ell_{1}, \ldots, \ell_{d-1}$ such that their pairwise differences are invertible, then the Diestel-Leader graph $\mathrm{DL}_{d}(q)$ is the Cayley graph of the group $\Gamma=\Gamma_{d}\left(\mathfrak{L}_{q}\right)$ of affine matrices

$$
\left(\begin{array}{cc}
\left(\mathrm{t}+\ell_{1}\right)^{k_{1}} \cdots\left(\mathrm{t}+\ell_{d-1}\right)^{k_{d-1}} & \mathrm{P} \\
0 & 1
\end{array}\right), \quad k_{1}, \ldots, k_{d-1} \in \mathbb{Z}, \mathrm{P} \in \mathcal{R}_{d}\left(\mathfrak{L}_{q}\right),
$$

with respect to the generators

$$
\begin{gathered}
\left(\begin{array}{cc}
\mathrm{t}+\ell_{i} & b \\
0 & 1
\end{array}\right)^{ \pm 1}, \quad b \in \mathfrak{L}_{q}, i \in\{1, \ldots, d-1\}, \\
\left(\begin{array}{cc}
\left(\mathrm{t}+\ell_{i}\right)\left(\mathrm{t}+\ell_{j}\right)^{-1} & b\left(\mathrm{t}+\ell_{j}\right)^{-1} \\
0 & 1
\end{array}\right), \quad b \in \mathfrak{L}_{q}, i, j \in\{1, \ldots, d-1\}, i \neq j .
\end{gathered}
$$

Proof. Each of $\mathfrak{L}_{q}\left(\left(\mathrm{t}+\ell_{i}\right)\right)(i=1, \ldots, d-1)$ and $\mathfrak{L}_{q}\left(\left(\mathrm{t}^{-1}\right)\right)$ is the completion of $\mathcal{R}_{d}\left(\mathfrak{L}_{q}\right)$ in its respective ultrametric. Indeed, for each $k \in \mathbb{Z}$ and distinct $i, j \in\{1, \ldots, d-1\}$, we can write $\left(\mathrm{t}+\ell_{j}\right)^{k}$ as a Laurent series in $\mathrm{t}+\ell_{i}$ as well as in $\mathrm{t}^{-1}$, namely

$$
\left(\mathrm{t}+\ell_{j}\right)^{k}=\sum_{n=0}^{\infty}\left(\begin{array}{l}
k \\
n
\end{array}\right)\left(\ell_{j}-\ell_{i}\right)^{k-n}\left(\mathrm{t}+\ell_{i}\right)^{n}=\sum_{n=-k}^{\infty}\left(\begin{array}{c}
k \\
k+n
\end{array}\right) \ell_{j}^{n+k_{\mathrm{t}}-n} .
$$

Also, for each non-negative $k \in \mathbb{Z}$ and $j \in\{1, \ldots, d-1\}$, we can write $\mathrm{t}^{k}$ as a polynomial in $\mathrm{t}+\ell_{j}$,

$$
\mathrm{t}^{k}=\sum_{n=0}^{k}\left(\begin{array}{l}
k \\
n
\end{array}\right)\left(-\ell_{j}\right)^{k-n}\left(\mathrm{t}+\ell_{j}\right)^{n}
$$

A straightforward computation shows that for all $k_{1}, \ldots, k_{d-1} \in \mathbb{Z}$,

$$
\begin{aligned}
& \mathrm{v}_{\ell_{i}}\left(\left(\mathrm{t}+\ell_{1}\right)^{k_{1}} \cdots\left(\mathrm{t}+\ell_{d-1}\right)^{k_{d-1}}\right)=k_{i}, \quad i=1, \ldots, d-1, \\
& \mathrm{v}_{\infty}\left(\left(\mathrm{t}+\ell_{1}\right)^{k_{1}} \cdots\left(\mathrm{t}+\ell_{d-1}\right)^{k_{d-1}}\right)=-k_{1}-\cdots-k_{d-1},
\end{aligned}
$$

summing up to 0 . In particular, it follows from 3.10 that the diagonal embedding

$$
\mathcal{R}_{d}\left(\mathfrak{L}_{q}\right) \hookrightarrow \prod_{i=1}^{d-1} \mathfrak{L}_{q}\left(\left(\mathrm{t}+\ell_{i}\right)\right) \times \mathfrak{L}_{q}\left(\left(\mathrm{t}^{-1}\right)\right),
$$

where $\mathrm{P} \mapsto\left(\mathrm{P}_{1}, \ldots, \mathrm{P}_{d}\right)$ with $\mathrm{P}_{i}=\mathrm{P}$ for all $i$, is discrete in this last direct product.

Following the above explanations, we can identify the vertices of the tree $\mathbb{T}_{q}$ with balls in the respective ultrametric. Thus, we get $d$ copies of $\mathbb{T}_{q}$. The first $d-1$ correspond to $\mathfrak{L}_{q}\left(\left(\mathrm{t}+\ell_{i}\right)\right) \equiv \partial^{*} \mathbb{T}_{q} \quad(i=1, \ldots, d-1)$, and recall that in each of these cases, we define the $n$-th horocycle to consist of all closed balls with radius $q^{-n}(n \in \mathbb{Z})$. The last copy of $\mathbb{T}_{q}$ corresponds to $\mathfrak{L}_{q}\left(\left(\mathrm{t}^{-1}\right)\right) \equiv \partial^{*} \mathbb{T}_{q}$, but also recall that this time, we define the $n$-th horocycle to consist of all closed balls with radius $q^{-n-1}$.

Each of the $d$ groups $\operatorname{Aff}\left(\mathfrak{L}_{q}\left(\left(\mathrm{t}+\ell_{i}\right)\right)\right), i=1, \ldots, d-1$, and $\operatorname{Aff}\left(\mathfrak{L}_{q}\left(\left(\mathrm{t}^{-1}\right)\right)\right)$ is a closed subgroup of $\operatorname{Aff}\left(\mathbb{T}_{q}\right)$ that acts transitively on the tree. By 3.11$)$, the diagonal embedding 
of $\Gamma_{d}\left(\mathfrak{L}_{q}\right)$ into their direct product is discrete. By 3.10 , combined with 3.3 , the action of $\Gamma_{d}\left(\mathfrak{L}_{q}\right)$ on $\left(\mathbb{T}_{q}\right)^{d}$ is such that the subgraph $\mathrm{DL}_{d}(q)$ is preserved. The last step in showing that $\mathrm{DL}_{d}(q)$ is a Cayley graph of our group consists in exhibiting a correspondence between graph and group that is bijective and compatible with the group action:

Claim. For all $k_{1}, \ldots, k_{d-1} \in \mathbb{Z}$, each $\mathrm{P} \in \mathcal{R}$ has a unique decomposition into Laurent polynomials $\mathrm{P}=\mathrm{P}_{k_{1}, \ldots, k_{d-1}}^{(1)}+\cdots+\mathrm{P}_{k_{1}, \ldots, k_{d-1}}^{(d)}$, where

$$
\begin{aligned}
& \mathrm{P}_{k_{1}, \ldots, k_{d-1}}^{(i)}=\sum_{n<k_{i}} a_{n, i}\left(\mathrm{t}+\ell_{i}\right)^{n}, \quad i=1, \ldots, d-1, \\
& \mathrm{P}_{k_{1}, \ldots, k_{d-1}}^{(d)}=\sum_{n \geq-\left(k_{1}+\cdots+k_{d-1}\right)} a_{n, d} \mathrm{t}^{n}
\end{aligned}
$$

with $a_{n, i} \in \mathfrak{L}_{q}$ and $\neq 0$ only for finitely many $n(i=1, \ldots, d)$.

Proof of the claim. By multiplying with $\left(\mathrm{t}+\ell_{1}\right)^{-k_{1}} \cdots\left(\mathrm{t}+\ell_{d-1}\right)^{-k_{d-1}}$, one sees that it is sufficient to prove this for $k_{1}=\cdots=k_{d}=0$. The existence of a decomposition 3.12) is an easy exercise which we leave to the reader. Uniqueness requires a bit more care. To this end, we have to prove that

$$
\mathrm{P}_{0, \ldots, 0}^{(1)}+\cdots+\mathrm{P}_{0, \ldots, 0}^{(d)}=0
$$

implies that $\mathrm{P}_{0, \ldots, 0}^{(i)}=0$ for all $i$. Suppose that for some $i \in\{1, \ldots, d-1\}$, we have $\mathrm{P}_{0, \ldots, 0}^{(i)} \neq 0$. Write $\mathrm{Q}$ for the left-hand side of 3.13 after subtracting $\mathrm{P}_{0, \ldots, 0}^{(i)}$. Then $\mathrm{v}_{\ell_{i}}\left(\mathrm{P}_{0, \ldots, 0}^{(i)}\right)<0$, but by 3.8 and 3.9 ,

$$
\mathrm{v}_{\ell_{i}}\left(\mathrm{P}_{0, \ldots, 0}^{(i)}\right)=\mathrm{v}_{\ell_{i}}(\mathrm{Q}) \geq 0
$$

a contradiction. Thus, $\mathrm{P}_{0, \ldots, 0}^{(i)}=0$ for $i=1, \ldots, d-1$, and consequently also for $i=d$, concluding the proof of the claim.

Our choice for the origin is now $o=o_{1} \cdots o_{d}$, where $o_{i}=B_{\ell_{i}}(0,1)$ for $i=1, \ldots, d-1$ and $o_{d}=B_{\infty}\left(0, q^{-1}\right)$. Let

$$
g=\left(\begin{array}{cc}
\left(\mathrm{t}+\ell_{1}\right)^{k_{1}} \cdots\left(\mathrm{t}+\ell_{d-1}\right)^{k_{d-1}} & \mathrm{P} \\
0 & 1
\end{array}\right) \in \Gamma_{d}(q) .
$$

In view of the claim we obtain a bijective correspondence $g \leftrightarrow x=x_{1} \cdots x_{d}=g o$, where

$$
\begin{aligned}
& x_{i}=g_{i} o_{i}=B_{\ell_{i}}\left(\mathrm{P}_{k_{1}, \ldots, k_{d-1}}^{(i)}, q^{-k_{i}}\right), \quad i=1, \ldots, d-1, \\
& x_{d}=g_{d} o_{d}=B_{\infty}\left(\mathrm{P}_{k_{1}, \ldots, k_{d-1}}^{(d)}, q^{k_{1}+\cdots+k_{d-1}-1}\right) .
\end{aligned}
$$

Here, when writing $g o$, we mean the image of $g$ under the diagonal embedding, while $g_{i} o_{i}$ refers again to $g_{i}=g$, but this time acting on the $i$-th tree of the horocyclic product: as isometries of $T_{i}=\mathbb{T}_{q}$, the $g_{i}$ are distinct.

Compatibility with the group action is straightforward: $(g h) o=g(h o)$ for all $g, h \in \Gamma$. 
Finally, using 3.3 , one checks that for each $b \in \mathfrak{L}_{q},\left(\begin{array}{cc}\mathrm{t}+\ell_{i} & b \\ 0 & 1\end{array}\right)$ maps the origin $o$ to one of its neighbours of type $\mathbf{e}_{i}-\mathbf{e}_{d}$, where $i \neq d$. Analogously, $\left(\begin{array}{cc}\left(\mathrm{t}+\ell_{i}\right)\left(\mathrm{t}+\ell_{j}\right)^{-1} & b\left(\mathrm{t}+\ell_{j}\right)^{-1} \\ 0 & 1\end{array}\right)$ maps $o$ to one of its neighbours of type $\mathbf{e}_{i}-\mathbf{e}_{j}$, where $i \neq j$.

We now study how we can implement Theorem 3.6 for specific values of $q$ and $d$.

\section{A. $\mathrm{DL}_{3}(q)$ and a finitely presented extension of the lamplighter group}

If $q \geq 2$ is arbitrary and $d=2$ (two trees) or $d=3$ (three trees), then we can use for $\mathfrak{L}_{q}$ the ring $\mathfrak{Z}_{q}$ of integers modulo $q$. Indeed, we may choose $\ell_{1}=0$ and $\ell_{2}=1$.

Thus, for the lamplighter group, we start with the $\operatorname{ring} \mathcal{R}_{2}\left(\mathfrak{Z}_{q}\right)=\mathfrak{Z}_{q}\left[\mathrm{t}^{-1}, \mathrm{t}\right]$ of all Laurent polynomials $\mathrm{P}=\mathrm{P}(\mathrm{t})=\sum_{k=K}^{L} a_{k} \mathrm{t}^{k}$ (with integers $K \leq L$ and $a_{k} \in \mathfrak{Z}_{q}$ ).

(3.14) Corollary. The graph $\mathrm{DL}_{2}(q)=\mathrm{DL}(q, q)$ is the Cayley graph of the lamplighter group $\mathfrak{Z}_{q} 2 \mathbb{Z}$, represented as the group $\Gamma_{2}\left(\mathfrak{Z}_{q}\right)$ of affine matrices

$$
\left(\begin{array}{ll}
\mathrm{t}^{k} & \mathrm{P} \\
0 & 1
\end{array}\right), \quad k \in \mathbb{Z}, \mathrm{P} \in \mathfrak{Z}_{q}\left[\mathrm{t}^{-1}, \mathrm{t}\right],
$$

with respect to the generators

$$
\left(\begin{array}{ll}
\mathrm{t} & b \\
0 & 1
\end{array}\right), \quad b \in \mathfrak{Z}_{q},
$$

and their inverses.

For $\mathrm{DL}_{3}(q)$, we use $\mathcal{R}_{3}\left(\mathfrak{Z}_{q}\right)=\mathfrak{Z}_{q}\left[\mathrm{t}^{-1},(\mathrm{t}+1)^{-1}, \mathrm{t}\right]$, which consists of all

$$
\mathrm{P}=\mathrm{P}(\mathrm{t}, \mathrm{t}+1)=\sum_{k=K}^{L} \sum_{m=M}^{N} a_{k, m} \mathrm{t}^{k}(\mathrm{t}+1)^{m},
$$

where $K, L, M, N \in \mathbb{Z}, K \leq L, M \leq N, a_{k, m} \in \mathfrak{Z}_{q}$.

(3.15) Corollary. The Diestel-Leader graph $\mathrm{DL}_{3}(q)=\mathrm{DL}(q, q, q)$ is the Cayley graph of the group $\Gamma=\Gamma_{3}\left(\mathfrak{Z}_{q}\right)$ of affine matrices

$$
\left(\begin{array}{cc}
\mathrm{t}^{k}(\mathrm{t}+1)^{m} & \mathrm{P} \\
0 & 1
\end{array}\right), \quad k, m \in \mathbb{Z}, \mathrm{P} \in \mathfrak{Z}_{q}\left[\mathrm{t}^{-1},(\mathrm{t}+1)^{-1}, \mathrm{t}\right],
$$

with respect to the generators

$$
\left(\begin{array}{ll}
\mathrm{t} & b \\
0 & 1
\end{array}\right),\left(\begin{array}{cc}
\mathrm{t}+1 & b \\
0 & 1
\end{array}\right),\left(\begin{array}{cc}
\mathrm{t}^{-1}+1 & b \mathrm{t}^{-1} \\
0 & 1
\end{array}\right), \quad b \in \mathfrak{Z}_{q},
$$

and their inverses.

The group $\Gamma_{3}\left(\mathfrak{Z}_{q}\right)$ is finitely presented (see Baumslag [4, 5] and also below in $\$ 4$ ). Thus, by Corollaries 3.14 and 3.15 we recover a concrete way of embedding the infinitely presented group $\mathfrak{Z}_{q}<\mathbb{Z}$ into the finitely presented, metabelian group $\Gamma_{3}\left(\mathfrak{Z}_{q}\right)$. 
How can we extend the above to $\mathrm{DL}_{d}(q)$ with larger $d$ ? If $d \geq 4$, we cannot always find $d-1$ elements $\ell_{i} \in \mathfrak{Z}_{q}$ whose pairwise differences are invertible. We note the following cases where we can still work with $\mathfrak{Z}_{q}$.

(i) When $q$ is odd and $d=4$ then we can use $\ell_{1}=0, \ell_{2}=1$ and $\ell_{3}=-1 \equiv q-1$ (modulo $q$ ). Thus, $\mathcal{R}_{4}\left(\mathfrak{Z}_{q}\right)=\mathfrak{Z}_{q}\left[\mathrm{t}^{-1},(\mathrm{t}+1)^{-1},(\mathrm{t}-1)^{-1}, \mathrm{t}\right]$, and $\mathrm{DL}_{4}(q)$ is a Cayley graph of the associated group of affine matrices.

(ii) When $q$ is prime and $d \leq q+1$ then we can use $\ell_{1}=0, \ldots, \ell_{d-1}=d-2$. Again, $\mathrm{DL}_{d}(q)$ is a Cayley graph of the group of affine matrices $\Gamma_{d}\left(\mathfrak{Z}_{q}\right)$ associated with $\mathcal{R}_{d}(q)=\mathfrak{Z}_{q}\left[\mathrm{t}^{-1},(\mathrm{t}+1)^{-1}, \ldots,(\mathrm{t}+d-2)^{-1}, \mathrm{t}\right]$.

The last case (where $\mathfrak{Z}_{q}=\mathbb{F}_{q}$, the field of order $q$ ) points towards a more general answer.

\section{B. $\mathrm{DL}_{d}(q)$ and quasi-isometries}

For arbitrary $q \geq 2$, let $q=p_{1} \cdots p_{r}$ be its factorization as a product of prime powers. (Caution: the $p_{\iota}$ are prime powers, not necessarily primes themselves!) Suppose first that $p_{\iota} \geq d-1$ for all $\iota \in\{1, \ldots, r\}$, and let $\mathbb{F}_{p_{\iota}}$ be the field of order $p_{\iota}$. Now construct the ring

$$
\mathbb{F}_{q}=\mathbb{F}_{p_{1}} \times \cdots \times \mathbb{F}_{p_{r}}
$$

with coordinatewise addition and multiplication. Let $\ell_{\iota, 1}, \ldots, \ell_{\iota, d-1}$ be distinct elements of each $\mathbb{F}_{p_{l}}$, and set $\ell_{j}=\left(\ell_{1, j}, \ldots, \ell_{r, j}\right) \in \mathbb{F}_{q}$ for $j=1, \ldots, d-1$. Then all the differences $\ell_{i}-\ell_{j} \quad(i \neq j)$ are invertible elements of $\mathbb{F}_{q}$. Therefore, we can apply Theorem 3.6 with the ring

$$
\mathcal{R}_{d}\left(\mathbb{F}_{q}\right)=\mathbb{F}_{q}\left[\left(\mathrm{t}+\ell_{1}\right)^{-1}, \ldots,\left(\mathrm{t}+\ell_{d-1}\right)^{-1}, \mathrm{t}\right]
$$

of all Laurent polynomials over $\mathbb{F}_{q}$ in $\mathrm{t}+\ell_{1}, \ldots, \mathrm{t}+\ell_{d-1}$.

(3.17) Corollary. Let $q=p_{1} \cdots p_{r}$ be the factorization of $q$ into prime powers, and suppose $p_{\iota} \geq d-1$ for all $\iota \in\{1, \ldots, r\}$. Then $\mathrm{DL}_{d}(q)$ is a Cayley graph of the group $\Gamma=\Gamma_{d}\left(\mathbb{F}_{q}\right)$ of affine matrices

$$
\left(\begin{array}{cc}
\left(\mathrm{t}+\ell_{1}\right)^{k_{1}} \cdots\left(\mathrm{t}+\ell_{d-1}\right)^{k_{d-1}} & \mathrm{P} \\
0 & 1
\end{array}\right), \quad k_{1}, \ldots, k_{d-1} \in \mathbb{Z}, \mathrm{P} \in \mathcal{R}_{d}\left(\mathbb{F}_{q}\right) .
$$

When $d \geq p_{\iota}$ for some $\iota \in\{1, \ldots, r\}$, then we cannot use the above construction. However, we can show that $\mathrm{DL}_{d}(q)$ is quasi-isometric with $\mathrm{DL}_{d}\left(q^{s}\right)$ for arbitrary $s \geq 1$, and when $s$ is sufficiently large, it is a Cayley graph by Corollary 3.17 .

Recall that a quasi-isometry between two metric spaces $\left(X_{1}, d_{1}\right)$ and $\left(X_{2}, d_{2}\right)$ is a mapping $\phi: X_{1} \rightarrow X_{2}$ such that there are constants $A>0, B \geq 0$ with

$$
\begin{aligned}
A^{-1} d_{2}\left(\phi x_{1}, \phi y_{1}\right)-B & \leq d_{1}\left(x_{1}, y_{1}\right) \leq A d_{2}\left(\phi x_{1}, \phi y_{1}\right)+B \quad \text { and } \\
d\left(x_{2}, \phi X_{1}\right) & \leq B \quad \text { for all } x_{1}, y_{1} \in X_{1}, x_{2} \in X_{2} .
\end{aligned}
$$


For connected graphs, quasi-isometry refers to the discrete graph metric, and for finitely generated groups, it refers to the word metric, i.e., the graph metric of one of its Cayley graphs: for a finitely generated group $\Gamma$, any two of its Cayley graphs with respect to finite, symmetric generating sets are quasi-isometric (with $B=0$, i.e., bi-Lipschitz). Quasi-isometry of groups has been an object of intensive study in geometric group theory since its introduction by Gromov [30]. For the following, recall the group $\mathcal{A}\left(q_{1}, \ldots, q_{d}\right)$ defined in Proposition 2.5

(3.19) Proposition. For arbitrary $q_{1}, \ldots, q_{d} \geq 2$ and $s \geq 2$, the graphs $\operatorname{DL}\left(q_{1}^{s}, \ldots, q_{d}^{s}\right)$ and $\operatorname{DL}\left(q_{1}, \ldots, q_{d}\right)$ are quasi-isometric. The quasi-isometry has the following property: if $\Gamma \leq \mathcal{A}\left(q_{1}, \ldots, q_{d}\right)$, then the subgroup

$$
\Gamma_{s}=\left\{g=g_{1} \cdots g_{d} \in \Gamma: \mathfrak{h}\left(g_{i}\right) \in s \mathbb{Z}, i=1, \ldots, d\right\}
$$

embeds into $\mathcal{A}\left(q_{1}^{s}, \ldots, q_{d}^{s}\right)$, and if $\Gamma$ acts transitively and/or with trivial stabilizers on $\operatorname{DL}\left(q_{1}, \ldots, q_{d}\right)$, then it also acts transitively and/or with trivial stabilizers on $\operatorname{DL}\left(q_{1}^{s}, \ldots, q_{d}^{s}\right)$.

Proof. In the tree $\mathbb{T}_{q_{i}}$, consider the horocycles $H_{s n}, n \in \mathbb{Z}$. Their union becomes the vertex set of $\mathbb{T}_{q^{s}}$, if we introduce new edges as follows: if $n \in \mathbb{Z}$ and $x \in H_{s n}$, then draw an edge between $x$ and $x^{-s} \in H_{s(n-1)}$, where $x^{-k}$ is defined recursively by $x^{-1}=x^{-}$ (the predecessor of $x$ ), and $x^{-k-1}=\left(x^{-k}\right)^{-}$. See Figure 4, where $q=2$ and $s=3$.

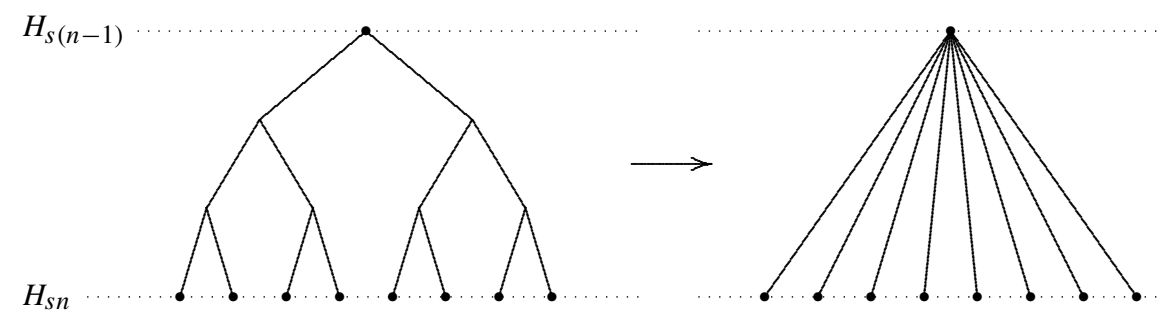

Fig. 4

In this way, by inverting the arrow in Figure 4, we get a mapping $\phi_{i}: \mathbb{T}_{q_{i}^{s}} \rightarrow \mathbb{T}_{q_{i}}$ which is one-to-one from the horocycle $H_{n}\left(\mathbb{T}_{q_{i}^{s}}\right)$ onto $H_{s n}\left(\mathbb{T}_{q_{i}}\right)$ for each $n \in \mathbb{Z}$. Furthermore,

$$
\begin{aligned}
& s \cdot(d(x, y)-2)+2 \leq d\left(\phi_{i} x, \phi_{i} y\right) \leq s \cdot d(x, y) \quad \text { and } \\
& \mathfrak{h}\left(\phi_{i} x\right)=s \cdot \mathfrak{h}(x) \quad \text { for all } x, y \in \mathbb{T}_{q_{i}^{s}} .
\end{aligned}
$$

(Here, $d(\cdot, \cdot)$ refers to the respective graph metric in each of the two graphs involved.) Clearly, $\phi_{i}$ is a quasi-isometry, and by 3.20), we can embed the group $\left\{g \in \operatorname{Aff}\left(\mathbb{T}_{q_{i}}\right)\right.$ : $\mathfrak{h}(g) \in s \mathbb{Z}\}$ into $\operatorname{Aff}\left(\mathbb{T}_{q_{i}^{s}}\right)$ by $g \mapsto \phi_{i}^{-1} \circ g \circ \phi_{i}$.

We now define $\phi: \operatorname{DL}\left(q_{1}^{s}, \ldots, q_{d}^{s}\right) \rightarrow \operatorname{DL}\left(q_{1}, \ldots, q_{d}\right)$ by $\phi=\phi_{1} \cdots \phi_{d}$, that is, the action of $\phi$ on the $i$-th coordinate $x_{i}$ of $x=x_{1} \cdots x_{d} \in \operatorname{DL}\left(q_{1}^{s}, \ldots, q_{d}^{s}\right)$ is given by $x_{i} \mapsto \phi_{i} x_{i}(i=1, \ldots, d)$. It is now straightforward that $\phi$ is a quasi-isometry with the asserted properties. 
(3.21) Corollary. For arbitrary $q$ and $d \geq 2$, the graph $\mathrm{DL}_{d}(q)$ is quasi-isometric with $\mathrm{DL}_{d}\left(q^{s}\right)$, which is a Cayley graph of the group $\Gamma_{d}\left(\mathbb{F}_{q^{s}}\right)$, when s is sufficiently large.

Proof. As above, let $q=p_{1} \cdots p_{r}$ be the factorization of $q$ into prime powers. Now choose $s$ sufficiently large such that $p_{\iota}^{s} \geq d-1$ for all $\iota \in\{1, \ldots, r\}$. Then $\mathrm{DL}_{d}\left(q^{s}\right)$ is a Cayley graph of the group $\Gamma_{d}\left(\mathbb{F}_{q^{s}}\right)$ by Corollary 3.17

(3.22) Remarks. (a) In conclusion, we are not able to prove that every Diestel-Leader graph $\mathrm{DL}_{d}(q)$ is itself a Cayley graph. The first open case is $\mathrm{DL}_{4}(2)$, the horocyclic product of four trees with the same branching number 2 (三 degree 3 ).

(b) Proposition 3.19 leads to the following question: is it true that two Diestel-Leader graphs are quasi-isometric if and only if there are $r, s \geq 1$ such that (up to permutation of their "coordinate" trees) they are of the form $\operatorname{DL}\left(q_{1}^{r}, \ldots, q_{d}^{r}\right)$ and $\operatorname{DL}\left(q_{1}^{s}, \ldots, q_{d}^{s}\right)$ ? For the case $d=2$, compare with Wortman [47, §4] and the results of Eskin, Fisher and Whyte [25].

(c) The following generalizes the conjecture of Diestel and Leader [23] that $\mathrm{DL}(2,3)$ is not quasi-isometric to any Cayley graph: is it true that $\mathrm{DL}\left(q_{1}, \ldots, q_{d}\right)$ for $d \geq 2$ is quasiisometric with a Cayley graph of some finitely generated group if and only if $q_{1}=\cdots$ $=q_{d}$ ? For $d=2$, see once more [25].

\section{The $D L$ complex}

We compute homotopical properties of DL in this section, and combine this information with the results of the previous section to derive conclusions on groups acting on DL.

We defined DL as a graph in the introduction; for our purposes, it is now better to view $\mathrm{DL}$ as a cell complex (in which cells are represented by subsets of the vertex set), whose 1-skeleton is the graph defined by (1.1) and (1.2).

Let therefore $T_{1}=\mathbb{T}_{q_{1}}, \ldots, T_{d}=\mathbb{T}_{q_{d}}$ be our homogeneous trees with their respective Busemann functions. We now turn $\mathrm{DL}=\mathrm{DL}\left(q_{1}, \ldots, q_{d}\right)$ into a cell complex, whose vertex set $\mathrm{DL}^{0}(0$-skeleton) is given by 1.1$\}$. Now choose for each $i \in\{1, \ldots, d\}$ a subset $E_{i} \subset X_{i}$ of cardinality 1 or 2 ; if $E_{i}=\{x, y\}$, then $x \sim y$ has to be an edge of $T_{i}$. To this choice there corresponds an $s$-dimensional cell

$$
\gamma\left(E_{1}, \ldots, E_{d}\right)=\left\{x_{1} \cdots x_{d} \in \mathrm{DL}^{0}: x_{i} \in E_{i} \text { for all } i\right\},
$$

whenever $\left|\gamma\left(E_{1}, \ldots, E_{d}\right)\right| \geq 2$, with $s=\left|E_{1}\right|+\cdots+\left|E_{d}\right|-d-1$. The faces of $\gamma\left(E_{1}, \ldots, E_{d}\right)$ are all $\gamma\left(E_{1}, \ldots, E_{j-1}, F_{j}, E_{j+1}, \ldots, E_{d}\right)$ for all possible choices of $j$ and $F_{j} \subset E_{j}$ with $\left|F_{j}\right|=1$ and $\left|E_{j}\right|=2$.

The 1-skeleton of DL is given by the condition (1.2), and the dimension of the DL complex is $d-1$. If $d=2$ then there are no cells of dimension $\geq 2$, and we recover the original construction of $\mathrm{DL}$ as a graph.

(4.1) Remark. A cell is always of the form $\gamma\left(E_{1}, \ldots, E_{d}\right)$, where each $E_{i}$ is either $\left\{x_{i}\right\}$ or $\left\{x_{i}, y_{i}\right\}$ with $x_{i} \sim y_{i}$ and $\mathfrak{h}\left(y_{i}\right)=\mathfrak{h}\left(x_{i}\right)+1$.

If $d \geq 3$ then besides the 1-dimensional cells (the edges), there are also two kinds of 2-dimensional cells: there must be precisely three indices $j<k<l$ such that 
$\left|E_{j}\right|=\left|E_{k}\right|=\left|E_{l}\right|=2$. The requirement that $\left|\gamma\left(E_{1}, \ldots, E_{d}\right)\right| \geq 2$ is met when $\sum \mathfrak{h}\left(x_{i}\right) \in\{-1,-2\}$. If now $\sum \mathfrak{h}\left(x_{i}\right)=-1$, then $\gamma\left(E_{1}, \ldots, E_{d}\right)$ is a triangle, with vertices $x_{1} \cdots x_{j-1} y_{j} x_{j+1} \cdots x_{d}, x_{1} \cdots x_{k-1} y_{k} x_{k+1} \cdots x_{d}$ and $x_{1} \cdots x_{l-1} y_{l} x_{l+1} \cdots x_{d}$. If $\sum \mathfrak{h}\left(x_{i}\right)=-2$, then $\gamma\left(E_{1}, \ldots, E_{d}\right)$ is also a triangle but of a different kind: its vertices have the form $x_{1} \cdots y_{j} \cdots y_{k} \cdots x_{d}, x_{1} \cdots y_{j} \cdots y_{l} \cdots x_{d}$ and $x_{1} \cdots y_{k} \cdots y_{l} \cdots x_{d}$.

If $d \geq 4$, then in addition, there are also three kinds of 3-dimensional cells: there is now a set $I$ of four indices with $\left|E_{i}\right|=2$ if $i \in I$, and we must have $\sum \mathfrak{h}\left(x_{i}\right) \in$ $\{-1,-2,-3\}$. If $\sum \mathfrak{h}\left(x_{i}\right)=-1$, then $\gamma\left(E_{1}, \ldots, E_{d}\right)$ is a tetrahedron, spanned by the vertices $z_{1} \cdots z_{d}$ with $z_{j}=y_{j}$ for precisely one $j \in I$, and all other $z_{i}=x_{i}$. If $\sum \mathfrak{h}\left(x_{i}\right)=-2$, then $\gamma\left(E_{1}, \ldots, E_{d}\right)$ is an octahedron, spanned by the vertices $z_{1} \cdots z_{d}$ with $z_{j}=y_{j}$ for precisely two $j \in I$ (there are $6=\left(\begin{array}{l}4 \\ 2\end{array}\right)$ choices). If $\sum \mathfrak{h}\left(x_{i}\right)=-3$, then $\gamma\left(E_{1}, \ldots, E_{d}\right)$ is again a tetrahedron, spanned by the vertices $z_{1} \cdots z_{d}$ with $z_{j}=y_{j}$ for precisely three $j \in I$.

In general, if $d \geq s+1$ there are $s$ kinds of $s$-dimensional cells, according to the values of $\sum \mathfrak{h}\left(x_{i}\right) \in\{-1, \ldots,-s\}$, and $\left|E_{i}\right|=2$ for all $i$ in a set $I \subset\{1, \ldots, d\}$ of cardinality $s+1$.

(4.2) Definition. Choose $R \in \mathbb{N}$ and points $b=b_{1} \cdots b_{d}, t=t_{1} \cdots t_{d}, t^{\prime}=t_{1}^{\prime} \cdots t_{d}^{\prime} \in$ $\prod_{i} T_{i}$ such that for all $i \in\{1, \ldots, d\}$ we have (a) $\sum_{i} \mathfrak{h}\left(b_{i}\right)=-R$, (b) $b_{i}=t_{i} \curlywedge t_{i}^{\prime}$ (the greatest common ancestor $)$, and (c) $\mathfrak{h}\left(t_{i}\right)=\mathfrak{h}\left(t_{i}^{\prime}\right)=\mathfrak{h}\left(b_{i}\right)+R$.

The octahedron $\mathcal{O}_{t, t^{\prime}, b}$ is the subcomplex of DL spanned by the vertices $x_{1} \cdots x_{d}$ such that $x_{i}$ lies on the path $\overline{t_{i} t_{i}^{\prime}}$ for all $i$.

The "downward" geodesics in $T_{i}$ of length $R$ starting at $b_{i}$ can be ordered lexicographically by the sequence ("word") of length $R$ of the labels (in $\left\{0, \ldots, q_{i}-1\right\}$ ) along their edges. The octahedron $\mathcal{O}_{t, t^{\prime}, b}$ is basic if for all $i$ the geodesic $\overline{b_{i} t_{i}^{\prime}}$ is the immediate successor of $\overline{b_{i} t_{i}}$, that is, the label words along $\overline{b_{i} t_{i}}$ and $\overline{b_{i} t_{i}^{\prime}}$ have the form $(j-1)\left(q_{i}-1\right) \cdots\left(q_{i}-1\right)$ and $j 0 \cdots 0$, respectively, where $j \in\left\{1, \ldots, q_{i}-1\right\}$. See Figure 5.
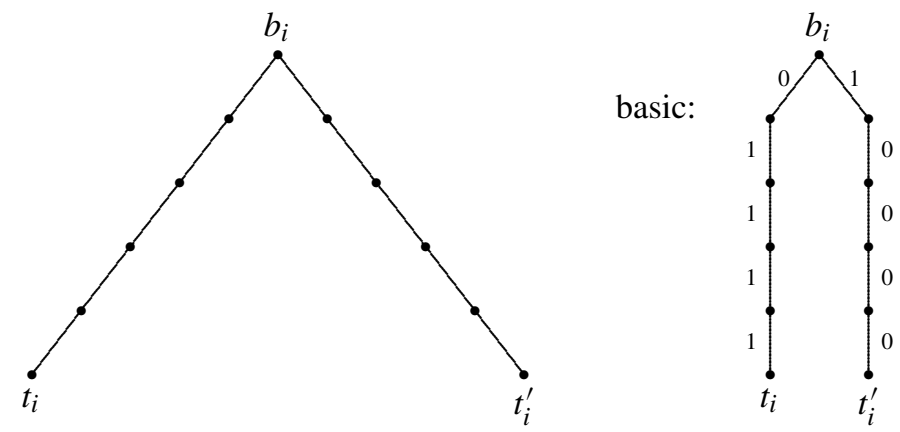

Fig. 5

If $d=3$, octahedra are usual octahedra: six extremal vertices and eight triangular faces with side lengths $R$. Each face is subdivided into basic triangles with side lengths 1 , that is, 2-cells; see Figure 6. For arbitrary $d$, octahedra have $2 d$ vertices and $2^{d}$ topdimensional faces. 


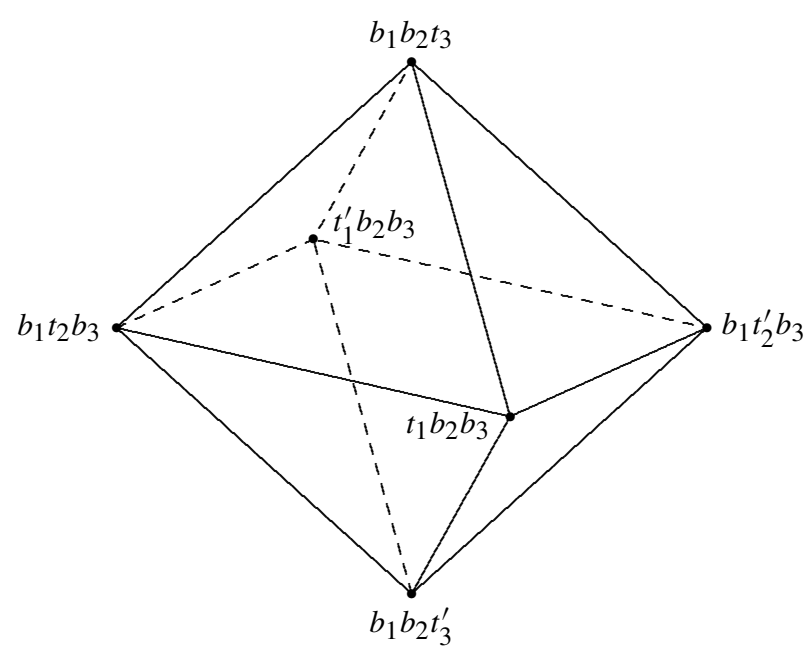

Fig. 6. A basic octahedron in $\operatorname{DL}\left(q_{1}, q_{2}, q_{3}\right)$. The face spanned by $b_{1} b_{2} t_{3}, b_{1} t_{2} b_{3}$ and $t_{1} b_{2} b_{3}$ consists of all $x_{1} x_{2} x_{3} \in \mathrm{DL}$ with $x_{i} \in \overline{b_{i} t_{i}}$.

(4.3) Lemma. Let $\mathfrak{l}_{i}, \mathfrak{l}_{i}^{\prime}$ be distinct geodesics from $\omega_{i}$ to the "lower" boundary $\partial^{*} T_{i}$ in $T_{i}$, and set $\mathcal{P}=\left(\mathfrak{l}_{1} \cup \mathfrak{l}_{1}^{\prime}\right) \times \cdots \times\left(\mathfrak{l}_{d} \cup \mathfrak{l}_{d}^{\prime}\right) \cap \mathrm{DL}$.

Let $b_{i}$ be the bifurcation point of $\mathfrak{l}_{i}$ and $\mathfrak{l}_{i}^{\prime}$; set $R=-\sum_{i} \mathfrak{h}\left(b_{i}\right)$; and let $t_{i}, t_{i}^{\prime}$ be the points respectively on $\mathfrak{l}_{i}, \mathfrak{l}_{i}^{\prime}$ with $\mathfrak{h}\left(t_{i}\right)=\mathfrak{h}\left(t_{i}^{\prime}\right)=\mathfrak{h}\left(b_{i}\right)+R$.

If $R>0$, then $\mathcal{P}$ retracts to the octahedron $\mathcal{O}_{t, t^{\prime}, b}$. If $R \leq 0$, then $\mathcal{P}$ is contractible.

Furthermore, every octahedron is homeomorphic to a sphere of dimension $d-1$.

Proof. Assume first $R>0$. Then clearly $\mathcal{O}=\mathcal{O}_{t, t^{\prime}, b}$ is a subset of $\mathcal{P}$. Consider a point $x=x_{1} \cdots x_{d}$ in $\mathcal{P} \backslash \mathcal{O}$. We will retract it to $\mathcal{O}$. Consider (1) in increasing order, the coordinates $i \in\{1, \ldots, d\}$ such that $\mathfrak{h}\left(x_{i}\right)<\mathfrak{h}\left(b_{i}\right)$ and, at the same time, (2) in decreasing order, the coordinates $j$ such that $\mathfrak{h}\left(x_{j}\right)>\mathfrak{h}\left(t_{i}\right)$. Move $x$, at unit speed, down on coordinate $i$ and up on coordinate $j$, until $x_{i}=b_{i}$ or $x_{j} \in\left\{t_{j}, t_{j}^{\prime}\right\}$; when this happens, move to the next $i$ or $j$. If there are no more $j$ 's available, keep moving up on the selected coordinate $j$.

This process defines a retraction, i.e., a continuous map $\rho: \mathcal{P} \times[0, \infty) \rightarrow \mathcal{P}$ with $\rho(x, 0)=x$ and $\lim _{t \rightarrow \infty} \rho(x, t) \in \mathcal{O}$.

If $R \leq 0$, then this process also produces a retraction, but now towards a single point in $\mathcal{P}$.

The last claim of the lemma is clear: an octahedron is topologically the $d$-fold join ${ }^{1}$ of a pair of points, that is, a $(d-1)$-dimensional sphere.

1 Recall that the join of two spaces $X, Y$ is $X \star Y=X \times[0,1] \times Y /\{(x, 0, y) \sim$ $\left.\left(x, 0, y^{\prime}\right),(x, 1, y) \sim\left(x^{\prime}, 1, y\right)\right\}$; it is classical that the join of an $(n-1)$-dimensional sphere and an $(m-1)$-dimensional sphere is an $(m+n-1)$-dimensional sphere. 
The following result bears a strong similarity to Theorem 4.1 of Bestvina and Brady [7]. However, there does not seem to be a natural discrete ambient group acting on $\prod T_{i}$ that would be required for their theorem to apply directly.

(4.4) Theorem. The Diestel-Leader graph $\mathrm{DL}\left(q_{1}, \ldots, q_{d}\right)$ has the homotopy type of a wedge of countably many $(d-1)$-spheres, that is, the topological space obtained by glueing together all those spheres at a single point. These spheres correspond bijectively to basic octahedra.

Proof. Embed each tree $T_{i}$ in the upper half-plane $\mathbb{H}$ in such a way that $\omega_{i}$ is the "upper" boundary point in $\mathbb{H}$, the "lower" boundary $\partial^{*} T_{i}$ lies in $\mathbb{R}$, and so that its edges are ordered lexicographically, i.e., the edges labelled $0,1, \ldots, q_{i}-1$ appear in left-to-right order below any vertex. Let $\Omega_{i}$ be the set of all geodesics in $T_{i}$ going from $\omega_{i}$ to $\mathbb{R}$.

The tree $T_{i}$, topologically, may be obtained from the disjoint union of the geodesics in $\Omega_{i}$ by glueing them along specified half-geodesics from a vertex to $\omega_{i}$. Therefore, the product $T_{1} \times \cdots \times T_{d}$ may be viewed as a disjoint union of hyperplanes glued along "hyper-octants", and is naturally embedded in $\mathbb{H}^{d}$.

Similarly, DL is obtained by considering the disjoint union of the planes $\mathfrak{l}_{1} \times \cdots \times$ $\mathfrak{l}_{d} \cap \mathrm{DL}$ for all $\mathfrak{l}_{i} \in \Omega_{i}$, and glueing them along subspaces. It is naturally embedded in a codimension-one contractible subspace $W$ of $\mathbb{H}^{d}$.

The homotopy type of DL, therefore, is that of a wedge of "pieces", which are glued together at a single point. Each "piece" is specified by two lexicographically consecutive geodesics $\mathfrak{l}_{i}, \mathfrak{l}_{i}^{\prime}$ in each tree $T_{i}$, and is of the form $\mathcal{P}=\left(\mathfrak{l}_{1} \cup \mathfrak{l}_{1}^{\prime}\right) \times \cdots \times\left(\mathfrak{l}_{d} \cup \mathfrak{l}_{d}^{\prime}\right) \cap \mathrm{DL}$. It is naturally a subspace of $W$. By Lemma 4.3 , this piece $\mathcal{P}$ is either contractible, or retracts to an octahedron, which is basic by the choice of $\mathfrak{l}_{i}, \mathfrak{l}_{i}^{\prime}$. Furthermore all basic octahedra appear in this way.

Finally, it is obvious that there are countably many basic octahedra, since there are countably many choices of $b_{i}, t_{i}$ and $t_{i}^{\prime}$.

Recall that a group $\Gamma$ is of type $F_{d}$ if it is the fundamental group of an aspherical cell complex (i.e. a $K(\Gamma, 1)$ ) whose $d$-skeleton is compact. ("Aspherical" means that it is a topological space whose fundamental cover is contractible.) In particular, "type $F_{1}$ " means "finitely generated", and "type $F_{2}$ " means "finitely presented".

(4.5) Corollary. If $\mathrm{DL}_{d}(q)$ is a Cayley graph of a group $\Gamma$, then $\Gamma$ is of type $F_{d-1}$, but not of type $F_{d}$. In particular, if $d \geq 2$, it is finitely generated, and if $d \geq 3$, it is finitely presented.

Proof. The cell complex $\Gamma \backslash \mathrm{DL}$ is compact, and by Theorem 4.4 it approximates up to dimension $d-1$ a classifying space $K(\Gamma, 1)$. This proves that $\Gamma$ is of type $F_{d-1}$. On the other hand, $K(\Gamma, 1)$ has as many $d$-cells as there are $\Gamma$-orbits on basic octahedra, and there are infinitely many of them, so $\Gamma$ is not of type $F_{d}$.

In particular, the group $\Gamma_{3}\left(\mathfrak{Z}_{q}\right)$ is finitely presented, and contains as a subgroup the lamplighter group $\Gamma_{2}\left(\mathfrak{Z}_{q}\right) \cong \mathfrak{Z}_{q} 2 \mathbb{Z}$. 
The first example of a finitely presented group that is not of type $F_{3}$ was discovered by Stallings [42]. It was only later, in particular through the work of Bieri [8], that Stallings' group was interpreted as the kernel of an epimorphism to $\mathbb{Z}$.

Explicit embeddings of the lamplighter group were already known: Johnson [31] embeds it first in the group $G$ of permutations of $\mathbb{Z}$ that are translations outside of a finite set (as the subgroup generated by the transposition $(1,2)$ and the translation $n \mapsto n+2$ ); and then naturally embeds $G$ in the group $\widetilde{G}$ of permutations of a three-branched star which are translations outside of a finite set; he finally exhibits a presentation of $\widetilde{G}$. (This group does not act transitively and with trivial stabilizers on $\mathrm{DL}_{3}(q)$.) Homological properties of some metabelian groups were studied by Bux [15], and the groups he considers are closely related to the matrix groups appearing in Theorem 3.6 .

The Cayley graph of $\Gamma_{3}\left(\mathfrak{Z}_{q}\right)$ contains unbounded "dead ends", as was first noticed by Cleary and Riley [21]. It is easy to see geometrical properties of this group in its DL description.

Baumslag [4, 5] proves that every finitely generated metabelian group can be embedded in a finitely presented metabelian group. His construction is quite explicit: the group into which $\mathfrak{Z}_{q}<\mathbb{Z}$ embeds has presentation

$$
\Gamma=\left\langle a, s, t \mid a^{q},[s, t],\left[a, a^{t}\right], a^{-t} a a^{s}\right\rangle .
$$

In fact, this presentation can be interpreted in terms of "lamplighters" quite naturally as follows.

Consider the abelian group $\mathfrak{V}$ of all finitely supported functions $\mathbb{Z}^{2} \rightarrow \mathfrak{Z}_{q}$ (the configurations of "lamps"). Thus, $\mathfrak{V}$ is generated by all point masses $\delta_{x, y}$ at $(x, y) \in \mathbb{Z}^{2}$ (with $\delta_{x, y}(x, y)=1 \in \mathfrak{Z}_{q}$ and 0 elsewhere). Let $\mathfrak{W}$ be the subgroup generated by all elements of the form $\delta_{x, y}+\delta_{x+1, y}-\delta_{x, y+1}$, where $(x, y) \in \mathbb{Z}^{2}$. The group $\mathbb{Z}^{2}=\langle s, t\rangle$ acts by translations on $\mathfrak{V}$ preserving $\mathfrak{W}$, and one has $\Gamma \cong\langle s, t\rangle \ltimes(\mathfrak{V} / \mathfrak{W})$, by identifying the element $a$ of the presentation 4.6 with $\delta_{0,0}$, and more generally $a^{s^{x}} t^{y}$ with $\delta_{x, y}$.

Using Corollary 3.15, it is easy to identify $\Gamma$ with $\mathrm{DL}_{3}(q)$, under the correspondence

$$
a \leftrightarrow\left(\begin{array}{ll}
1 & 1 \\
0 & 1
\end{array}\right), \quad s \leftrightarrow\left(\begin{array}{cc}
\mathrm{t}^{-1} & 0 \\
0 & 1
\end{array}\right), \quad t \leftrightarrow\left(\begin{array}{cc}
(1+\mathrm{t})^{-1} & 0 \\
0 & 1
\end{array}\right) .
$$

We also remark that in our context it may be more adequate to replace $\mathbb{Z}^{2}$ with $\mathbb{A}_{2}$ via the correspondence $(x, y) \mapsto(x, y,-x-y)$.

More generally, a presentation of $\Gamma_{d}(q)$ may be read off from DL, as follows.

(4.7) Theorem. Under the hypotheses and with the notation of Corollary 3.17, if $d \geq 3$, the group $\Gamma_{d}(q)$ admits the presentation

$$
\begin{aligned}
\Gamma_{d}(q)=\left\langle g_{i, j, \lambda}, 1 \leq i, j \leq d(i \neq j), \lambda \in \mathfrak{Z}_{q}\right| g_{i, j, \lambda} g_{j, i,-\lambda}, \\
\quad g_{j, i, \lambda} g_{k, j, \mu} g_{i, k, v} \text { whenever } \lambda+\mu+v=0, \\
g_{i, j, \lambda} g_{j, k, \mu} g_{k, i, v} \text { whenever } \\
\lambda+\mu+v=0 \text { and } \lambda \ell_{k}+\mu \ell_{i}+v \ell_{j}=0 \text { and } d \neq i, j, k, \\
\left.\quad g_{i, j, \lambda} g_{j, d, \mu} g_{d, i, v} \text { whenever } \mu+v=0 \text { and } \lambda+\mu \ell_{i}+v \ell_{j}=0\right\rangle .
\end{aligned}
$$


Proof. By Theorem 4.4, the 2-skeleton of $\mathrm{DL}$ is simply connected if $d \geq 3$. Therefore a presentation of $\Gamma_{d}(q)$ may be read off from this 2 -skeleton. The generators are the $\Gamma_{d}(q)$-orbits of edges, and the relations are the $\Gamma_{d}(q)$-orbits of 2-cells. These orbits can be identified with the neighbourhood of a given vertex. There are $d(d-1) q$ generators, since this last number is the vertex degree in $\mathrm{DL}_{d}(q)$.

The group element $g_{i, j, \lambda}$ maps $o$ to its neighbour $x$ of type $\mathbf{e}_{i}-\mathbf{e}_{j}$ with label $\lambda$, that is, $x_{j}=o_{j}^{-}, x_{i}^{-}=o_{i}$, and the label on the edge $\left[o_{i}, x_{i}\right]$ of $T_{i}$ is $\lambda$. Thus, the inverse of $g_{i, j, \lambda}$ is $g_{j, i,-\lambda}$. This corresponds to the relations in line (a).

There are $d(d-1)(d-2) q^{2}$ relations of length 3 corresponding to the first kind of triangles indicated in Remark 4.1, these are given by the relations in line (b). There are also $d(d-1)(d-2) q$ relations of length 3 corresponding to the second kind of triangles indicated in Remark 4.1, these are given by the relations in lines (c) and (d).

Note that the generators $g_{i, d, \lambda}$ and $g_{i, j, \lambda}$ seem to have different roles in the above presentation. This asymmetry can be masked as follows: define polynomials $\Lambda_{i}=\mathrm{t}+\ell_{i}$ for $i<d$, and $\Lambda_{d}=1$. Then the presentation can be written as

$$
\begin{aligned}
\Gamma_{d}(q)=\langle & g_{i, j, \lambda}, 1 \leq i, j \leq d(i \neq j), \lambda \in \mathfrak{Z}_{q} \mid g_{i, j, \lambda} g_{j, i,-\lambda}, \\
& g_{j, i, \lambda} g_{k, j, \mu} g_{i, k, v} \text { whenever } \lambda+\mu+v=0, \\
& \left.g_{i, j, \lambda} g_{j, k, \mu} g_{k, i, v} \text { whenever } \lambda \Lambda_{k}+\mu \Lambda_{i}+v \Lambda_{j}=0\right\rangle .
\end{aligned}
$$

Moreover, combining with Corollary 4.5, we obtain:

(4.8) Corollary. The lamplighter group $\mathfrak{Z}_{q} 2 \mathbb{Z}$ can, for all $d$, be embedded in a metabelian group of type $F_{d}$.

Proof. We will use some algebraic number theory in the proof.

Factor $q=p_{1} \cdots p_{r}$ as a product of prime powers. It suffices to embed $\mathfrak{Z}_{p_{\iota}} 2 \mathbb{Z}$ in a metabelian group of type $F_{d}$. Indeed, $\mathfrak{Z}_{q} \cong \mathfrak{Z}_{p_{1}} \times \cdots \times \mathfrak{Z}_{p_{r}}$, so that by identifying $\mathbb{Z}$ with the principal diagonal of $\mathbb{Z}^{r}$ via $k \leftrightarrow(k, \ldots, k)$, we can embed $\mathfrak{Z}_{q} 2 \mathbb{Z}$ into the direct product of the $\mathfrak{Z}_{p_{\iota}}<\mathbb{Z}$. But type $F_{d}$ is inherited by products.

Write therefore $p_{\iota}=p^{n}$ as a prime power, and let $s$ be such that $p^{s} \geq d$. The field $\mathbb{F}_{p^{s}}$ contains elements $\ell_{1}^{\prime}, \ldots, \ell_{d}^{\prime}$ whose pairwise differences are invertible.

There exists a unique non-ramified extension $\mathbb{K}$ of $\mathbb{Q}_{p}$, with ring of integers $\mathfrak{O}$ and maximal ideal $\mathcal{M}=p \mathfrak{O}$, such that $\mathfrak{O} / \mathcal{M} \cong \mathbb{F}_{p^{s}}$. Furthermore, $\mathbb{K}$ is a $\mathbb{Q}_{p}$-vector space of dimension $s$, and $\mathfrak{O}$ is a free $\mathbb{Z}_{p}$-module of rank $s$. For all $i$, let $\ell_{i}^{\prime \prime}$ be an arbitrary preimage of $\ell_{i}^{\prime}$ in $\mathfrak{O}$ under the natural projection $\mathfrak{O} \rightarrow \mathbb{F}_{p^{s}}$. Then the pairwise differences $\ell_{i}^{\prime \prime}-\ell_{j}^{\prime \prime}$ lie in $\mathfrak{O} \backslash \mathcal{M}$, and are therefore invertible in $\mathfrak{O}$.

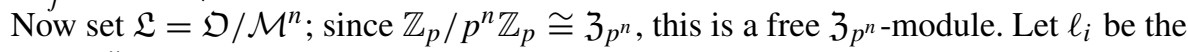
image of $\ell_{i}^{\prime \prime}$ in $\mathfrak{L}$. The pairwise differences of the different $\ell_{i}$ are again invertible in $\mathfrak{L}$.

In the group $\Gamma_{d+1}(\mathfrak{L})$ - which has type $F_{d}$ by Corollary $4.5-$ consider the elements $x=\left(\begin{array}{ll}1 & 1 \\ 0 & 1\end{array}\right)$ and $y=\left(\begin{array}{cc}\mathrm{t}+\ell_{1} & 0 \\ 0 & 1\end{array}\right)$. Then $x$ has order $p^{n}$, and the subgroup $\langle x, y\rangle$ of $\Gamma_{d+1}(\mathfrak{L})$ is isomorphic to $\mathfrak{Z}_{p^{n}} \geq \mathbb{Z}$.

Note that it is not possible to embed $\mathfrak{Z}_{q} 2 \mathbb{Z}$ in a metabelian group of type $F$ (i.e., one with a compact $K(\Gamma, 1)$ ). Indeed, recall the following from Bieri and Groves [9]: a group $G$ is of 
type $F P_{n}$, for $n \in \mathbb{N} \cup\{\infty\}$, if the trivial $G$-module $\mathbb{Z}$ admits a $\mathbb{Z} G$-projective resolution of bounded rank in each dimension up to $n$; this condition is weaker than type $F_{n}$. A group $G$ is of type $F P$ if it is of type $F P_{\infty}$ and has finite cohomological dimension; this condition is weaker than type $F$, but it also implies that $G$ is torsion-free. In [9] it is proven that a metabelian group of type $F P_{\infty}$ is virtually of type $F P$, and therefore is virtually torsionfree.

(4.9) Remark. Assume that $\Gamma$ is one of the groups with Cayley graph $\mathrm{DL}_{d}(q)$, constructed in $\$ 3$. Then $\Gamma$ is in most cases an "automata group", as we shall explain below. But it is never an "automatic group" in the sense of Epstein et al. [24]. Indeed, by Corollary 4.5. $\Gamma$ is of type $F_{d-1}$ but not of type $F_{d}$; in particular, it is not of type $F P_{\infty}$. On the other hand, automatic groups are always of type $F P_{\infty}$, by [24, Theorem 10.2.6].

We briefly recall the definition of automata groups. Fix an alphabet $\Sigma$. Automata groups are permutation groups of the set of infinite words $\Sigma^{\infty}$. A family of automatic transformations is given by a machine with bounded memory computing the permutation in real time. It can be modelled by a finite set $A$ of states and a function $\Phi: A \times \Sigma \rightarrow$ $\Sigma \times A$ such that for all $a \in A$ the composition $((\tau, b) \mapsto \tau) \circ \Phi \circ(\sigma \mapsto(a, \sigma))$ is a permutation of $\Sigma$. The associated transformations $T_{a}$, for $a \in A$, act on infinite strings $\sigma=\sigma_{1} \sigma_{2} \cdots \in \Sigma^{\infty}$ by

$$
T_{a}(\sigma)=\tau_{1} T_{b}\left(\sigma_{2} \sigma_{3} \cdots\right) \quad \text { if } \Phi\left(a, \sigma_{1}\right)=\left(\tau_{1}, b\right)
$$

The product and inverse of automatic transformations are easily seen to be automatic.

Assume now that the group $\Gamma=\Gamma_{d}\left(\mathfrak{L}_{q}\right)$ is constructed as in Theorem 3.6 by use of elements $\ell_{1}, \ldots, \ell_{d-1} \in \mathfrak{L}_{q}$ whose pairwise differences are invertible. Assume furthermore that all $\ell_{i} \in \mathfrak{L}_{q}$ are invertible. Identify $\Sigma$ with $\mathfrak{L}_{q}$, and $\Sigma^{\infty}$ with $\mathfrak{L}_{q}[[\mathrm{t}]]$, the ring of formal power series in tover $\mathfrak{L}_{q}$.

For $j \in\{1, \ldots, d-1\}$, consider the finite set of states $A^{(j)} \cong \mathfrak{L}_{q}$ with associated affine transformations $T_{a}^{(j)}$ of $\Sigma^{\infty} \equiv \mathfrak{L}_{q}[[\mathrm{t}]]$ of the form

$$
T_{a}^{(j)}(\mathrm{f}(\mathrm{t}))=a+\left(\mathrm{t}+\ell_{j}\right) \mathrm{f}(\mathrm{t}), \quad \text { where } \quad a \in \mathfrak{L}_{q} .
$$

Note here that invertibility of $\ell_{j}$ implies that also the inverse transformation of $T_{a}^{(j)}$ preserves $\mathfrak{L}_{q}[[\mathrm{t}]]$. If we write $\mathrm{f}(\mathrm{t})=b_{0}+\operatorname{tg}(\mathrm{t})$ with constant term $b_{0} \in \mathfrak{L}_{q}$ and $\mathrm{g}(\mathrm{t}) \in \mathfrak{L}_{q}[[\mathrm{t}]]$, then

$$
T_{a}^{(j)}(\mathrm{f}(\mathrm{t}))=\left(\ell_{j} b_{0}+a\right)+\mathrm{t} \cdot T_{b_{0}}^{(j)}(\mathrm{g}(\mathrm{t})),
$$

and therefore each $T_{a}^{(j)}$ is an automatic transformation, with

$$
\Phi_{j}(a, b)=\left(\ell_{j} b+a, b\right) .
$$

Since $\Gamma$ is generated by $\left\{T_{a}^{(j)}: j=1, \ldots, d-1, a \in \mathfrak{L}_{q}\right\}$, all of its elements are automatic transformations. We note again that in the construction above we need $\ell_{j}$ to be invertible. The inverse of $T_{a}^{(j)}$ is again defined by an automaton: let us write $\left(T_{a}^{(j)}\right)^{-1}=$ $U_{a}^{(j)}$; then we have $U_{a}^{(j)}\left(b_{0}+\operatorname{tg}(\mathrm{t})\right)=\ell_{j}^{-1}\left(b_{0}-a\right)+\mathrm{t} \cdot U_{\ell_{j}^{-1}\left(b_{0}-a\right)}^{(j)}(\mathrm{g}(\mathrm{t}))$, so $U_{a}^{(j)}$ is the 
transformation defined on the set of states $\bar{A}^{(j)} \cong \mathfrak{L}_{q}$ by the map $\bar{\Phi}(a, b)=\left(\ell_{j}^{-1}(b-a)\right.$, $\left.\ell_{j}^{-1}(b-a)\right)$.

For more general information on automata groups, see the survey by Bartholdi, Grigorchuk and Nekrashevych [2].

We finally remark that $\operatorname{DL}\left(q_{1}, \ldots, q_{d}\right)$ has rational growth function $\sum_{x \in \mathrm{DL}} \mathrm{d}^{d(x, o)}$, where $d(\cdot, \cdot)$ is the graph metric. We intend to come back to this point on another occasion.

\section{The spectrum of simple random walk}

Recall the definition 1.5, 1.6 of the SRW operator $P$. It acts on the space $\ell^{2}(\mathrm{DL})$ of all square-summable functions $f: \mathrm{DL} \rightarrow \mathbb{C}$ with the standard inner product $\langle f, g\rangle=$ $\sum_{x} f(x) \overline{g(x)}$. Since $P$ is stochastic and self-adjoint, we have $\|P\|=\rho(P) \leq 1$, where $\rho(P)$ is its spectral radius. We set $\rho^{\prime}(P)=\min \operatorname{spec}(P)$, and write $D=(d-1) \sum_{i} q_{i}$ for the (constant) vertex degree of DL.

\section{A. Polyhedra and horizontal functions}

We now generalize the method of [3], where the spectrum of SRW on the horocyclic product of two trees was considered.

A function $f: \mathrm{DL} \rightarrow \mathbb{R}$ is called horizontal if it is finitely supported and

$$
\sum_{y_{j} \in \mathbb{T}_{j}: \mathfrak{h}\left(y_{j}\right)=\mathfrak{h}\left(x_{j}\right)} f\left(x_{1} \cdots x_{j-1} y_{j} x_{j+1} \cdots x_{d}\right)=0
$$

for every $x=x_{1} \cdots x_{d} \in \mathrm{DL}$ and $j \in\{1, \ldots, d\}$.

(5.2) Lemma. The subspace of $\ell^{2}(\mathrm{DL})$ spanned by the horizontal functions is dense.

Proof. Since the point masses $\delta_{x}(x \in \mathrm{DL})$ generate a dense subspace of $\ell^{2}(\mathrm{DL})$, and since $\mathrm{DL}$ is vertex-transitive, it is sufficient to show that $\delta_{o}$ (with $o=o_{1} \cdots o_{d}$ ) can be approximated in the $\ell^{2}$-norm by horizontal functions.

For each $j$, pick a vertex $b_{j}=b_{j}^{n}$ of $T_{j}$ on the horocycle $H_{-n}^{j}$ of $T_{j}$ that is not an ancestor of $o_{j}$. Now let $f_{j}=f_{j}^{(n)}$ be the function on $T_{j}$ defined by

$$
f_{j}\left(x_{j}\right)= \begin{cases}1 & \text { if } x_{j}=o_{j}, \\ -q_{j}^{-n} & \text { if } b_{j} \preccurlyeq x_{j} \in H_{0}^{j}, \\ 0 & \text { in all other cases. }\end{cases}
$$

Let $f=f^{(n)}$ be defined by $f\left(x_{1} \cdots x_{d}\right)=f_{1}\left(x_{1}\right) \cdots f_{d}\left(x_{d}\right)$. This function is horizontal, and

$$
\left\|f^{(n)}-\delta_{o}\right\|^{2}=\prod_{j=1}^{d}\left(q_{j}^{-n}+1\right)-1 \rightarrow 0 \quad \text { as } n \rightarrow \infty .
$$


(5.3) Definition. Let $a_{1}, \ldots, a_{d}$ be vertices of $T_{1}, \ldots, T_{d}$, respectively, such that $N=$ $-\sum_{j=1}^{d} \mathfrak{h}\left(a_{j}\right) \geq 2$. The polyhedron $\mathrm{S}=\mathrm{S}\left(a_{1}, \ldots, a_{d}\right)$ is the induced subgraph of $\mathrm{DL}$ on the set

$$
\left\{x_{1} \cdots x_{d} \in \mathrm{DL}: a_{j} \preccurlyeq x_{j} \forall j=1, \ldots, d\right\}
$$

with height $N=N(\mathrm{~S})$.

Note that $\mathrm{S}$ is finite; the octahedra $\mathcal{O}$ of Definition 4.2 are unions of $2^{d}$ polyhedra of the same height. Set

$$
\begin{aligned}
\mathbf{h} & =\mathbf{h}(\mathrm{S})=\left(\mathfrak{h}\left(a_{1}\right), \ldots, \mathfrak{h}\left(a_{d}\right)\right) \in \mathbb{Z}^{d}, \\
B_{\mathbf{h}} & =\left\{\mathbf{k} \in \mathbb{A}_{d-1}: k_{j} \geq \mathfrak{h}\left(a_{j}\right) \text { for } j=1, \ldots, d\right\} .
\end{aligned}
$$

If $\mathrm{S}$ and $\widetilde{\mathrm{S}}$ are two polyhedra with the same height $N=N(\mathrm{~S})=N(\widetilde{\mathrm{S}})$, then the graphs $B_{\mathbf{h}(\mathrm{S})}$ and $B_{\mathbf{h}(\widetilde{\mathrm{S}})}$ are isomorphic. As a representative of their isomorphism class, we single out the following one, by a slight abuse of notation:

$$
B_{N}=B_{(0, \ldots, 0,-N)}=\left\{\mathbf{k} \in \mathbb{A}_{d-1}: k_{1}, \ldots, k_{d-1} \geq 0, k_{d} \geq-N\right\} .
$$

It contains $\left(\begin{array}{c}N+d-1 \\ d-1\end{array}\right)$ elements. If $x=x_{1} \cdots x_{d}$ is an element of $\mathrm{S}\left(a_{1}, \ldots, a_{d}\right)$ and $\mathfrak{H}(x)=$ $\left(\mathfrak{h}\left(x_{1}\right), \ldots, \mathfrak{h}\left(x_{d}\right)\right)$ then $\mathfrak{H}(x)-\mathbf{h}-N \mathbf{e}_{d} \in B_{N}$. In particular, for each $j$, we have $x_{j} \in$ $T\left(a_{j}\right)=\left\{y_{j} \in T_{j}: a_{j} \preccurlyeq y_{j}\right\}$ and $d\left(x_{j}, a_{j}\right) \leq N$. The boundary $\partial \mathrm{S}$ of $\mathrm{S}$, that is, the set of all points in $\mathrm{S}$ having a neighbour in $\mathrm{DL} \backslash \mathrm{S}$, consists of all points $x \in \mathrm{S}$ for which there is at least one $j$ such that $x_{j}=a_{j}$. The interior of $\mathrm{S}$ is $\mathrm{S}^{o}=\mathrm{S} \backslash \partial \mathrm{S}$. Analogously, we set $\partial B_{\mathbf{h}}=\left\{\mathbf{k} \in B_{\mathbf{h}}: k_{j}=\mathfrak{h}\left(a_{j}\right)\right.$ for some $\left.j\right\}$ and $B_{\mathbf{h}}^{o}=B_{\mathbf{h}} \backslash \partial B_{\mathbf{h}}$, the boundary and interior of the graph $B_{\mathbf{h}}$ (cf. Fig. 7).

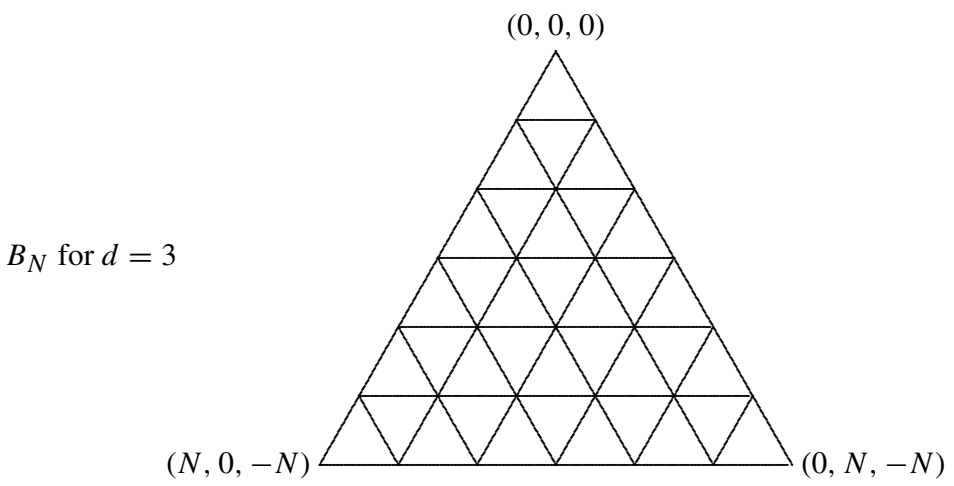

Fig. 7

For $\mathbf{k} \in B_{\mathbf{h}}$, the $\mathbf{k}$-th level of $\mathbf{S}$ is defined by

$$
L_{\mathbf{k}}=L_{\mathbf{k}}\left(a_{1}, \ldots, a_{d}\right)=\left\{x \in \mathrm{S}: \mathfrak{h}\left(x_{j}\right)=k_{j}, j=1, \ldots, d\right\}=\prod_{j=1}^{d}\left(T_{j}\left(a_{j}\right) \cap H_{k_{j}}^{j}\right),
$$

where $H_{m}^{j}$ denotes the $m$-th horocycle of the tree $T_{j}$. As in Figure 1, we can label all edges of $T_{j}$ by the elements of $\mathfrak{Z}_{q_{j}}$ so that for each vertex of $T_{j}$, the edges to its successors all 
carry distinct labels. Write $v_{j, l}$ for the successor of $a_{j}$ such that the edge from $a_{j}$ to $v_{j, l}$ has label $l \in \mathfrak{Z}_{q_{j}}$. We choose a function $\varphi^{j}: \mathfrak{Z}_{q_{j}} \rightarrow \mathbb{R}$ with

$$
\sum_{l \in \mathfrak{Z}_{q_{j}}} \varphi^{j}(l)=0 \quad \text { and } \quad \sum_{l \in \mathfrak{Z}_{q_{j}}}\left(\varphi^{j}(l)\right)^{2}=1 .
$$

For $j \in\{1, \ldots, d\}$ and $k \in \mathbb{Z}$, we denote by $f_{k}^{j}=f_{k}^{j}\left[a_{j}, \varphi^{j}\right]: T_{j} \rightarrow \mathbb{R}$ the function

$$
f_{k}^{j}\left(x_{j}\right)= \begin{cases}\varphi^{j}(l) q^{\left(\mathfrak{h}\left(a_{j}\right)-k+1\right) / 2} & \text { if } k>\mathfrak{h}\left(a_{j}\right) \text { and } v_{j, l} \preccurlyeq x_{j} \in H_{k}^{j}, \\ 0 & \text { otherwise, }\end{cases}
$$

and we define for each $\mathbf{k}=\left(k_{1}, \ldots, k_{d}\right) \in \mathbb{A}_{d-1}$ the following function on $\mathrm{DL}$ :

$$
f_{\mathbf{k}, \mathrm{S}}(x)=f_{\mathbf{k}}\left[\mathrm{S}, \varphi^{1}, \ldots, \varphi^{d}\right](x)=\prod_{j=1}^{d} f_{k_{j}}^{j}\left(x_{j}\right), \quad x=x_{1} \cdots x_{d} \in \mathrm{DL} .
$$

Since $f_{\mathbf{k}, \mathrm{S}} \equiv 0$ on $\mathrm{DL} \backslash \mathrm{S}^{o}$, we can also consider $f_{\mathbf{k}, \mathrm{S}}$ as a function on $\mathrm{S}=\mathrm{S}\left(a_{1}, \ldots, a_{d}\right)$ which is 0 on $\partial S$.

(5.8) Lemma. The functions $f_{\mathbf{k}, \mathrm{S}}$, where $\mathbf{k} \in B_{\mathbf{h}}^{o}$ and $\mathbf{h}=\mathbf{h}(\mathrm{S})$, are horizontal and orthonormal in $\ell^{2}(\mathrm{DL})$, and

$$
P f_{\mathbf{k}, \mathrm{S}}=\frac{1}{D} \sum_{i=1}^{d} \sum_{j \neq i} \sqrt{q_{i} q_{j}} f_{\mathbf{k}+\mathbf{e}_{i}-\mathbf{e}_{j}, \mathrm{~S}} .
$$

Proof. It is immediate by construction that the $f_{\mathbf{k}, S}, \mathbf{k} \in B_{\mathbf{h}}^{o}$, are horizontal and orthonormal. Regarding the action of $P$, first note that for each $x_{j} \in T_{j}$, we have

$$
\sum_{y_{j}: y_{j}^{-}=x_{j}} f_{k}^{j}\left(y_{j}\right)=\sqrt{q_{j}} f_{k-1}^{j}\left(x_{j}\right),
$$

and when $x_{j}^{-} \neq a_{j}$ then also $f_{k}^{j}\left(x_{j}^{-}\right)=\sqrt{q_{j}} f_{k+1}^{j}\left(x_{j}\right)$. Thus, for $x \in \mathrm{DL} \backslash \partial \mathrm{S}$ we have

$$
\begin{aligned}
P f_{\mathbf{k}, \mathbf{S}}(x) & =\frac{1}{D} \sum_{i=1}^{d} \sum_{j \neq i} \sum_{y \in N_{j, i}(x)} f_{\mathbf{k}, \mathbf{S}}(y) \\
& =\frac{1}{D} \sum_{i=1}^{d} f_{k_{i}}^{i}\left(x_{i}^{-}\right) \sum_{j \neq i}\left(\sum_{y_{j} \in T_{j}: y_{j}^{-}=x_{j}} f_{k_{j}}^{j}\left(y_{j}\right)\right) \prod_{l \neq i, j} f_{k_{l}}^{l}\left(x_{l}\right) \\
& (\text { using now that } x \notin \partial \mathrm{S}) \\
& =\frac{1}{D} \sum_{i=1}^{d} \sqrt{q_{i}} f_{k_{i}+1}^{i}\left(x_{i}\right) \sum_{j \neq i} \sqrt{q_{j}} f_{k_{j}-1}^{j}\left(x_{j}\right) \prod_{l \neq i, j} f_{k_{l}}^{l}\left(x_{l}\right) \\
& =\frac{1}{D} \sum_{i=1}^{d} \sum_{j \neq i} \sqrt{q_{i} q_{j}} f_{\mathbf{k}+\mathbf{e}_{i}-\mathbf{e}_{j}, \mathrm{~S}}(x) .
\end{aligned}
$$


When $x \in \partial \mathrm{S}$, there is $j^{\prime}$ such that $x_{j^{\prime}}=a_{j^{\prime}}$. Thus, the double sum in the last line vanishes. On the other hand, we split the sum in the second line into three pieces. The first is the sum over all pairs $(i, j)$ with $i \neq j$ and both $i, j \neq j^{\prime}$. Then $\prod_{l \neq i, j} f_{k_{l}}^{l}\left(x_{l}\right)=0$, since this product contains the factor $f_{k_{j^{\prime}}}^{j^{\prime}}\left(a_{j^{\prime}}\right)=0$. The second is the sum over all pairs $(i, j)$ with $i \neq j^{\prime}$ and $j=j^{\prime}$. Then $\sum_{y_{j}^{-}=x_{j}} f_{k_{j}}^{j}\left(y_{j}\right)=0$ since $x_{j^{\prime}}=a_{j^{\prime}}$. The third is the sum over all pairs $(i, j)$ with $i=j^{\prime}$ and $j \neq j^{\prime}$. But then $f_{k_{i}}^{i}\left(x_{i}^{-}\right)=f_{k_{j^{\prime}}}^{j^{\prime}}\left(a_{j^{\prime}}^{-}\right)=0$. Thus, we also have $P f_{\mathbf{k}, \mathrm{S}}(x)=0$ when $x \in \partial \mathrm{S}$.

(5.9) Corollary. If $q_{1}=\cdots=q_{d}=q$ then for all $\mathbf{k} \in B_{N}^{o}$,

$$
P f_{\mathbf{k}, \mathrm{S}}=\frac{1}{d(d-1)} \sum_{i=1}^{d} \sum_{j \neq i} f_{\mathbf{k}+\mathbf{e}_{i}-\mathbf{e}_{j}, \mathrm{~S}}
$$

\section{B. The spectra of $Q$ and $P$}

Lemma 5.8 leads us to consider the self-adjoint convolution operator on $\ell^{2}\left(\mathbb{Z}^{d}\right)$ defined by

$$
Q f(\mathbf{k})=\frac{1}{D} \sum_{i=1}^{d} \sum_{j \neq i} \sqrt{q_{i} q_{j}} f\left(\mathbf{k}+\mathbf{e}_{i}-\mathbf{e}_{j}\right) .
$$

It leaves the subspace $\ell^{2}\left(\mathbb{A}_{d-1}\right)$ invariant.

(5.11) Proposition. The spectrum of $Q$ is an interval $\left[\rho^{\prime}(Q), \rho(Q)\right]$ with endpoints $\rho(Q)=\sum_{i, j: j \neq i} \sqrt{q_{i} q_{j}} / D$ and $\rho^{\prime}(Q) \geq-1 /(d-1)$.

(i) If there is an $i$ such that $\sqrt{q_{i}}>\sum_{j \neq i} \sqrt{q_{j}}$ then $\rho^{\prime}(Q)>-1 /(d-1)$.

(ii) If $d=2$ then $\rho^{\prime}(Q)=-\rho(Q)$.

(iii) If for each $i$ there is $j \neq i$ such that $q_{i}=q_{j}$ then $\rho^{\prime}(Q)=-1 /(d-1)$.

(iv) In particular, if $q_{1}=\cdots=q_{d}$ then $\operatorname{spec}(Q)=[-1 /(d-1), 1]$.

Proof. For $\mathbf{t}=\left(t_{1}, \ldots, t_{d}\right) \in[0,2 \pi]^{d}$, the conjugate of the operator $Q$ by the Fourier transform is the operator of multiplication with the function

$$
\widehat{Q}(\mathbf{t})=\frac{1}{D} \sum_{i, j: j \neq i} \sqrt{q_{i} q_{j}} \cos \left(t_{i}-t_{j}\right) .
$$

It is well known that $\operatorname{spec}(Q)$ coincides with the set of values of $\widehat{Q}$. As a continuous image of the connected set $[0,2 \pi]^{d}$, it must be an interval. The upper bound $\rho(Q)$ is attained for $\mathbf{t}=\mathbf{0}$. For the lower bound, rewrite

$$
\widehat{Q}(\mathbf{t})=\frac{1}{D}\left(\left|\sum_{j=1}^{d} \sqrt{q_{j}} e^{i} t_{j}\right|^{2}-\sum_{j=1}^{d} q_{j}\right) \geq-\frac{1}{D} \sum_{j=1}^{d} q_{j}=-\frac{1}{d-1} .
$$

(Here, $\mathfrak{i}$ is the complex unit.) 
In case (i), we have

$$
\left|\sum_{j=1}^{d} \sqrt{q_{j}} e^{i t_{j}}\right| \geq \sqrt{q_{i}}-\sum_{j \neq i} \sqrt{q_{j}}>0,
$$

whence inf $\widehat{t} \widehat{Q}(\mathbf{t})>-1 /(d-1)$.

In case (ii), we find $\widehat{Q}(\pi, 0)=-\rho(Q)$.

In case (iii), suppose without loss of generality that there are $2 \leq r(1) \leq \cdots \leq$ $r(s)=d$ such that $r(l)-r(l-1) \geq 2$ and $q_{j}=q_{r(l)}$ for $r(l-1)<j \leq r(l)$. For those $j$, we set $t_{j}=2 \pi j /(r(l)-r(l-1))$, so that $\sum_{j=r(l-1)+1}^{r(l)} e^{\mathfrak{i} t_{j}}=0$, whence $\widehat{Q}\left(t_{1}, \ldots, t_{d}\right)=-1 /(d-1)$.

Now consider the vector space $\mathfrak{V}_{0}\left(B_{\mathbf{h}}\right)$ of all functions $f: B_{\mathbf{h}} \rightarrow \mathbb{C}$ with $f \equiv 0$ on $\partial B_{\mathbf{h}}$, and the symmetric operator (matrix) $Q_{\mathbf{h}}: \mathfrak{V}_{0}\left(B_{\mathbf{h}}\right) \rightarrow \mathfrak{V}_{0}\left(B_{\mathbf{h}}\right)$ defined by

$$
Q_{\mathbf{h}} f(\mathbf{k})= \begin{cases}Q f(\mathbf{k}) & \text { if } \mathbf{k} \in B_{\mathbf{h}}^{o}, \\ 0 & \text { if } \mathbf{k} \in \partial B_{\mathbf{h}} .\end{cases}
$$

Again by slight abuse of notation, we write $Q_{N}$ for $Q_{(0, \ldots, 0,-N)}$, acting on $\mathfrak{V}_{0}\left(B_{N}\right)$. Since $B_{\mathbf{h}}$ and $B_{N}$ are isomorphic when $\langle\mathbf{h}, \mathbf{1}\rangle=-N$, we have $\operatorname{spec}\left(Q_{\mathbf{h}}\right)=\operatorname{spec}\left(Q_{N}\right)$ in this case.

(5.12) Lemma.

$$
\operatorname{spec}(Q)=\overline{\bigcup_{N \geq 2} \operatorname{spec}\left(Q_{N}\right)}
$$

Proof. The operator $Q$ is the simple random walk operator on $\mathbb{A}_{d-1}$. In particular, $\operatorname{spec}(Q)$ is the spectrum of $Q$ acting on $\ell^{2}\left(\mathbb{A}_{d-1}\right)$.

Consider functions in $\mathfrak{V}_{0}\left(B_{N}\right)$ as functions on $\mathbb{A}_{d-1}$ with value 0 outside of $B_{N}^{o}$. For each $f \in \mathfrak{V}_{0}\left(B_{N}\right)$, we have $Q_{N} f(\mathbf{k})=Q f(\mathbf{k})$ for each $\mathbf{k} \in \mathbb{A}_{d-1} \backslash \partial B_{N}$, while $f \equiv 0$ on $\partial B_{N}$. Therefore

$$
\left\langle Q_{N} f, f\right\rangle=\langle Q f, f\rangle\left\{\begin{array}{l}
\leq \rho(Q)\langle f, f\rangle \\
\geq \rho^{\prime}(Q)\langle f, f\rangle .
\end{array}\right.
$$

Consequently, $\rho^{\prime}(Q) \leq \rho^{\prime}\left(Q_{N}\right) \leq \rho\left(Q_{N}\right) \leq \rho(Q)$, and

$$
\operatorname{spec}\left(Q_{N}\right) \subset\left[\rho^{\prime}(Q), \rho(Q)\right]=\operatorname{spec}(Q) .
$$

On the other hand, choose $\lambda \in \operatorname{spec}(Q)$ and $\varepsilon>0$. As $Q$ is self-adjoint there exists a finitely supported $f \in \ell^{2}\left(\mathbb{A}_{d-1}\right)$ of norm 1 such that $\|\lambda f-Q f\|<\varepsilon$. There is an $\mathbf{h}$ such that $f \in \mathfrak{V}_{0}\left(B_{\mathbf{h}}\right)$ and $Q f=Q_{\mathbf{h}} f$. Thus, expanding $f$ with respect to an orthonormal basis of $\mathfrak{V}_{0}\left(B_{\mathbf{h}}\right)$ consisting of $Q_{\mathbf{h}}$-eigenfunctions, we see that there must be an eigenvalue $\lambda^{\prime}$ of $Q_{\mathbf{h}}$ such that $\left|\lambda-\lambda^{\prime}\right|<\varepsilon$. Since $\operatorname{spec}\left(Q_{\mathbf{h}}\right)=\operatorname{spec}\left(Q_{N}\right)$, where $N=-\langle\mathbf{h}, \mathbf{1}\rangle$, the proof is complete.

(5.13) Lemma. Let $\mathrm{S}=\mathrm{S}\left(a_{1}, \ldots, a_{d}\right)$ and $\widetilde{\mathrm{S}}=\mathrm{S}\left(\tilde{a}_{1}, \ldots, \tilde{a}_{d}\right)$ be two polyhedra, both of height $\geq 2$, and set $\mathbf{h}=\mathbf{h}(\mathrm{S}), \tilde{\mathbf{h}}=\mathbf{h}(\widetilde{S})$. Furthermore let $\varphi^{1}, \ldots, \varphi^{d}$ and $\widetilde{\varphi}^{1}, \ldots, \widetilde{\varphi}^{d}$ be functions on $\mathfrak{Z}_{q_{j}}$ satisfying $\sqrt[5.5]{ }$. For $\mathbf{k} \in B_{\mathbf{h}}^{o}$ and $\mathbf{l} \in B_{\widetilde{\mathbf{h}}}^{o}$, write $f_{\mathbf{k}, S}=f_{\mathbf{k}}\left[\mathrm{S}, \varphi^{1}, \ldots, \varphi^{d}\right]$ and $\tilde{f}_{\mathbf{l}, \widetilde{S}}=f_{\mathbf{l}}\left[\widetilde{S}, \widetilde{\varphi}^{1}, \ldots, \widetilde{\varphi}^{d}\right]$. Suppose that one of the following conditions is satisfied. 
(1) $\left(a_{1}, \ldots, a_{d}\right) \neq\left(\tilde{a}_{1}, \ldots, \tilde{a}_{d}\right)$, or

(2) $\left(a_{1}, \ldots, a_{d}\right)=\left(\tilde{a}_{1}, \ldots, \tilde{a}_{d}\right)$ and $\varphi^{j} \perp \widetilde{\varphi}^{j}$ in $\ell^{2}\left(\mathfrak{Z}_{q_{j}}\right)$ for some $j \in\{1, \ldots, d\}$.

Then

$$
\left\{f_{\mathbf{k}, \mathrm{S}}: \mathbf{k} \in B_{\mathbf{h}}^{o}\right\} \perp\left\{\tilde{f}_{\mathbf{l}, \widetilde{\mathrm{s}}}: \mathbf{l} \in B_{\widetilde{\mathbf{h}}}^{o}\right\} .
$$

Proof. (1) If $\mathrm{S} \cap \widetilde{\mathrm{S}}=\emptyset$ then the above two sets are obviously orthogonal.

Suppose now $\mathrm{S} \cap \widetilde{\mathrm{S}} \neq \emptyset$. There is $j$ such that $a_{j} \neq \tilde{a}_{j}$, and $a_{j}, \tilde{a}_{j}$ are comparable in the ancestor relation $\preccurlyeq$. Without loss of generality, suppose $a_{1} \neq \tilde{a}_{1}$ and $a_{1} \preccurlyeq \tilde{a}_{1}$. Then $\tilde{a}_{1} \succcurlyeq v_{1, l}$, where the latter is one of the successors of $a_{1}$ in $T_{1}$.

Let $\mathbf{k} \in B_{N}^{o}$ and $\mathbf{l} \in B_{\tilde{N}}^{o}$ be given. If $\mathbf{k} \neq \mathbf{l}$ then certainly $f_{\mathbf{k}, S} \perp \widetilde{f}_{\mathbf{l}, \widetilde{S}}$, as these functions are supported on disjoint sets.

Assume therefore $\mathbf{k}=\mathbf{1}$. By construction 5.6, the function $f_{k_{1}}^{1}$ on the subtree of $T_{1}$ is constant on the support of $\widetilde{f}_{k_{1}}^{1}$. Also by construction, the sum of $\widetilde{f}_{k_{1}}^{1}$ over its support is zero. Thus,

$$
\sum_{x_{1} \in T_{1}: \mathfrak{h}\left(x_{1}\right)=k_{1}} f_{k_{1}}^{1}\left(x_{1}\right) \tilde{f}_{k_{1}}^{1}\left(x_{1}\right)=0,
$$

that is, $f_{k_{1}}^{1} \perp \widetilde{f}_{k_{1}}^{1}$. Hence, again by construction 5.6,

$$
\sum_{x} f_{\mathbf{k}, \mathbf{S}}(x) \tilde{f}_{\mathbf{l}, \widetilde{\mathbf{S}}}(x)=\prod_{j} \sum_{x_{j}} f_{k_{j}}^{j}\left(x_{j}\right) \tilde{f}_{k_{j}}^{j}\left(x_{j}\right)=0 .
$$

(2) If $\left(a_{1}, \ldots, a_{d}\right)=\left(\tilde{a}_{1}, \ldots, \tilde{a}_{d}\right)$ then $\widetilde{\mathrm{S}}=\mathrm{S}$ and $\tilde{\mathbf{h}}=\mathbf{h}$. If $\mathbf{k}, \mathbf{l} \in B_{N}^{o}$ are distinct then $f_{\mathbf{k}, \mathrm{S}}$ and $\tilde{f}_{\mathbf{l}, \mathrm{S}}$ have disjoint supports and are perpendicular. In the last remaining case, we compute

$$
\left\langle f_{\mathbf{k}, \mathrm{S}}, \widetilde{f}_{\mathbf{k}, \mathrm{S}}\right\rangle=\prod_{j=1}^{d}\left\langle\varphi^{j}, \widetilde{\varphi}^{j}\right\rangle=0 .
$$

Now let $\left\{\psi_{\mathbf{m}, N}: \mathbf{m} \in B_{N}^{o}\right\}$ be an orthonormal basis of $\mathfrak{V}_{0}\left(B_{n}\right)$ consisting of eigenfunctions (-vectors) of $Q_{N}$ with associated eigenvalues $\lambda_{\mathbf{m}, N}$, parametrized by all $\mathbf{m} \in B_{N}^{o}$. The following is immediate from Lemma 5.8 and the fact that for $\langle\mathbf{h}, \mathbf{1}\rangle=-N$, the natural isomorphism $B_{N} \rightarrow B_{\mathbf{h}}$ is given by $\mathbf{k} \mapsto \mathbf{k}+\mathbf{h}+N \mathbf{e}_{d}$.

(5.14) Corollary. Let $\mathrm{S}=\mathrm{S}\left(a_{1}, \ldots, a_{d}\right)$, $\mathbf{h}=\mathbf{h}(\mathrm{S})$ and $N=N(\mathrm{~S})$. The functions $g_{\mathbf{m}, \mathrm{S}}: \mathrm{DL} \rightarrow \mathbb{R}$, defined by

$$
g_{\mathbf{m}, \mathrm{S}}=\sum_{\mathbf{k} \in B_{N}^{o}} \psi_{\mathbf{m}, N}(\mathbf{k}) f_{\mathbf{k}+\mathbf{h}+N \mathbf{e}_{d}, \mathrm{~S}}, \quad \mathbf{m} \in B_{N}^{o},
$$

are orthonormal and horizontal vectors in $\ell^{2}(\mathrm{~S})$ as well as in $\ell^{2}(\mathrm{DL})$. They satisfy

$$
\operatorname{span}\left\{g_{\mathbf{m}, \mathrm{S}}: \mathbf{m} \in B_{N}^{o}\right\}=\operatorname{span}\left\{f_{\mathbf{k}, \mathrm{S}}: \mathbf{k} \in B_{\mathbf{h}}^{o}\right\} \quad \text { and } \quad P g_{\mathbf{m}, \mathrm{S}}=\lambda_{\mathbf{m}, N} \cdot g_{\mathbf{m}, \mathrm{S}} .
$$


Remember that each function $g_{\mathbf{m}, S}$, with $\mathbf{m} \in B_{N}^{o}$, depends not only on the polyhedron $\mathrm{S}=\mathrm{S}\left(a_{1}, \ldots, a_{d}\right)$, but also on the functions $\varphi_{1}, \ldots, \varphi_{d}$ of (5.5), that is,

$$
g_{\mathbf{m}, \mathbf{S}}=g_{\mathbf{m}}\left[\mathbf{S}, \varphi_{1}, \ldots, \varphi_{d}\right] \text {. }
$$

For each $q_{j}$, we now select functions $\varphi_{k}^{j}, k \in \mathfrak{Z}_{q_{j}} \backslash\{0\}$, that satisfy 5.5 and are mutually orthogonal:

$$
\varphi_{l}^{j}(s)= \begin{cases}0, & s=0, \ldots, l-2, \\ \left(q_{j}-l\right) / \sqrt{\left(q_{j}-l\right)\left(q_{j}+1-l\right)}, & s=l-1, \\ -1 / \sqrt{\left(q_{j}-l\right)\left(q_{j}+1-l\right)}, & s=l, \ldots, q_{j}-1 .\end{cases}
$$

(5.17) Proposition. The set

$$
\mathfrak{B}_{\mathrm{S}}=\left\{g_{\mathbf{m}}\left[\widetilde{\mathrm{S}}, \varphi_{l_{1}}^{1}, \ldots, \varphi_{l_{d}}^{d}\right]: \widetilde{\mathrm{S}} \subset \mathrm{S}, \mathbf{m} \in B_{N(\widetilde{\mathrm{S}})}^{o}, l_{j} \in \mathfrak{Z}_{q_{j}} \backslash\{0\}\right\},
$$

where $\widetilde{\mathrm{S}}$ runs through all polyhedra with height $N(\widetilde{\mathrm{S}}) \geq 2$ contained in $\mathrm{S}$, is an orthonormal basis of the vector space $\mathfrak{V}_{0} \mathrm{~S}$ of all functions which are horizontal and supported by $\mathrm{S}$.

Proof. Lemma 5.13 and Corollary 5.14 imply that the elements of $\mathfrak{B}_{S}$ are orthonormal, hence linearly independent. What remains is to show that $\mathfrak{B}_{S}$ spans the whole of $\mathfrak{V}_{0}(\mathrm{~S})$. We can replace each $g_{\mathbf{m}}\left[\widetilde{\mathrm{S}}, \varphi_{l_{1}}^{1}, \ldots, \varphi_{l_{d}}^{d}\right] \in \mathfrak{B}_{S}$ with $f_{\mathbf{m}+\mathbf{h}+N \mathbf{e}_{d}}\left[\widetilde{\mathrm{S}}, \varphi_{l_{1}}^{1}, \ldots, \varphi_{l_{d}}^{d}\right]$, since these last functions are also linearly independent and span the same vector space by Corollary 5.14

Recall that $L_{\mathbf{k}}$ denotes the k-th level of the polyhedron $\mathbf{S}$ with height $N=N(\mathrm{~S})$. A function $f$ in the space of all complex functions supported by $L_{\mathbf{k}}$ is horizontal if and only if it satisfies each of the equations

$$
\sum_{y_{j} \in \mathbb{T}_{j}: \mathfrak{h}\left(y_{j}\right)=\mathfrak{h}\left(x_{j}\right)} f\left(x_{1} \cdots x_{j-1} y_{j} x_{j+1} \cdots x_{d}\right)=0,
$$

for all $j \in\{1, \ldots, d\}$ and $x_{i} \in T_{i}\left(a_{i}\right) \cap H_{k_{i}}^{j}$ for each $i \neq j$. The number of these equations is $\sum_{j=1}^{d} \prod_{i \neq j} q_{i}^{k_{i}-\mathfrak{h}\left(a_{i}\right)}$, but they are not independent. By inclusion-exclusion, we find

$$
\operatorname{dim} \mathfrak{V}_{0}\left(L_{\mathbf{k}}\right)=\prod_{i=1}^{d}\left(q_{i}^{k_{i}-\mathfrak{h}\left(a_{i}\right)}-1\right)
$$

The proof will be complete when we can show that $\mathfrak{V}_{0}\left(L_{\mathbf{k}}\right)$ is spanned by all the functions $f_{\mathbf{k}}\left[\widetilde{\mathrm{S}}, \varphi_{l_{1}}^{1}, \ldots, \varphi_{l_{d}}^{d}\right]$, where $\widetilde{\mathrm{S}} \subset \mathrm{S}$, which are supported in $L_{\mathbf{k}}$. To this end, we count all functions of this last type (which are linearly independent).

First of all, the interior of $\widetilde{\mathrm{S}}=\mathrm{S}\left(\tilde{a}_{1}, \ldots, \tilde{a}_{d}\right)$ has to intersect $L_{\mathbf{k}}$. This means that for each $j$, we need to have $\mathfrak{h}\left(a_{j}\right) \leq \mathfrak{h}\left(\tilde{a}_{j}\right)<k_{j}$. There are $\sum_{r=0}^{k_{j}-\mathfrak{h}\left(a_{j}\right)-1} q_{j}^{r}$ points $\tilde{a}_{j}$ of this type. Thus, the number of feasible polyhedra $\widetilde{\mathrm{S}}$ is $\prod_{j=1}^{d}\left(q_{j}^{k_{j}-\mathfrak{h}\left(a_{j}\right)}-1\right) /\left(q_{j}-1\right)$. 
The remaining choices that we have are those of the functions $\varphi_{l}^{j}$, where $l \in \mathfrak{Z}_{q_{j}} \backslash$ $\{0\}$ and $j \in\{1, \ldots, d\}$. There are $\prod_{j=1}^{d}\left(q_{j}-1\right)$ such choices, so that the total number of functions $f_{\mathbf{m}}\left[\widetilde{\mathrm{S}}, \varphi_{l_{1}}^{1}, \ldots, \varphi_{l_{d}}^{d}\right]$ supported in $L_{\mathbf{k}}$ is $\prod_{j=1}^{d}\left(q_{j}^{k_{j}-\mathfrak{h}\left(a_{j}\right)}-1\right)$. This number coincides with the dimension of $\mathfrak{V}_{0}\left(L_{\mathbf{k}}\right)$, as claimed.

We can now show that as sets, the spectra of $P$ on $\ell^{2}(\mathrm{DL})$ and $Q$ on $\mathbb{A}_{d-1}$ coincide, although their "inner structure" is completely different. Indeed, $\operatorname{spec}(Q)$ is continuous, i.e., the associated spectral (Plancherel) measure is absolutely continuous with respect to Lebesgue measure, while for $\operatorname{spec}(P)$, we have the following.

(5.18) Theorem. The spectrum of the operator $P$ is pure point. It is the closure of the set of eigenvalues

$$
\operatorname{spec}_{p}(P)=\left\{\lambda_{\mathbf{m}, N}: N \geq 2, \mathbf{m} \in B_{N}^{o}\right\}=\bigcup_{N \geq 2} \operatorname{spec}\left(Q_{N}\right) .
$$

Each eigenvalue has infinite multiplicity. An associated orthonormal basis consisting of finitely supported eigenfunctions of $P$ is given by

$\mathfrak{B}=\left\{g_{\mathbf{m}}\left[\mathrm{S}, \varphi_{l_{1}}^{1}, \ldots, \varphi_{l_{d}}^{d}\right]: \mathrm{S}\right.$ polyhedron in $\mathrm{DL}$ with $\left.n(\mathrm{~S}) \geq 2, \mathbf{m} \in B_{N(\mathrm{~S})}^{o}, l_{j} \in \mathfrak{Z}_{q_{j}} \backslash\{0\}\right\}$,

where $g_{\mathbf{m}}\left[\mathrm{S}, \varphi_{l_{1}}^{1}, \ldots, \varphi_{l_{d}}^{d}\right]$ is defined in Corollary 5.14 and 5.15.

Proof. Pick any function $f$ on DL which is horizontal. Its support is then contained in an appropriate polyhedron S. Proposition 5.17 implies that $f$ is in the span of $\mathfrak{B}_{\mathrm{S}}$. Hence every horizontal function is a linear combination of functions in $\mathfrak{B}$. Therefore $\mathfrak{B}$ is an orthonormal basis by Lemma 5.2 and the whole spectrum must consist of the closure of the set of associated eigenvalues.

In particular, we get $\rho(P)=\rho(Q)$, that is, the spectral radius of $P$ is

$$
\rho(P)=\frac{2}{D} \sum_{i, j: i<j} \sqrt{q_{i} q_{j}}, \quad \text { where } \quad D=(d-1) \sum_{i} q_{i} .
$$

This formula might also have been derived in a completely different way, by use of Theorem $1(\mathrm{~b})$ of [41].

For $\operatorname{DL}\left(q_{1}, q_{2}\right)$, the eigenvalues of $P$ can be computed explicitly [3]. In general, this amounts to the explicit computation of the eigenvalues of $Q_{N}$ on $B_{N}$. For $d \geq 3$, this is the precise discrete analogue to computing the eigenvalues of the Laplacian on the $(d-1)$-dimensional Euclidean simplex whose side lengths are proportional to $\sqrt{q_{i} q_{j}}$ (respectively). An explicit solution to this last problem is known only for equilateral triangles in dimension $d-1=2$, but in no other case: this solution goes back to the work of Lamé in the 19th century (see e.g. Lamé [33]), and has reappeared in the literature several times (with or without knowledge of Lamé's work): see Pinsky [38], Práger [39. 40], McCartin [36]. 
C. The spectrum of $P$ on $\operatorname{DL}(q, q, q)$

We now consider briefly the case of $\operatorname{DL}(q, q, q)$, where we know from Corollary 5.9 that $Q$ is the transition operator of SRW on $\mathbb{A}_{2}$.

Let $\mathfrak{S}_{3}$ be the permutation group of $\{1,2,3\}$. For $\sigma \in \mathfrak{S}_{3}$ and $\mathbf{k}=\left(k_{1}, k_{2}, k_{3}\right) \in \mathbb{Z}^{3}$, we write $\sigma \mathbf{k}=\left(k_{\sigma^{-1}(1)}, k_{\sigma^{-1}(2)}, k_{\sigma^{-1}(3)}\right)$. Also, we write $\mathfrak{A}_{3}$ for the subgroup of even permutations in $\mathfrak{S}_{3}$. Now we define the following functions for $N \geq 2$ :

$$
\psi_{\mathbf{m}, N}(\mathbf{k})=\frac{1}{\sqrt{6} N} \sum_{\sigma \in \mathfrak{S}_{3}} \operatorname{sign}(\sigma) \exp \left(\operatorname{sign}(\sigma) \frac{2 \pi \mathfrak{i}}{3 N}\langle\mathbf{m}, \sigma \mathbf{k}\rangle\right), \quad \mathbf{m}, \mathbf{k} \in \mathbb{A}_{2} .
$$

(5.21) Proposition. We have the following:

$$
\begin{aligned}
\psi_{\mathbf{m}, N} & =0 & & \text { on } \partial B_{N} \text { for each } \mathbf{m} \in B_{N}^{o}, \\
\left\langle\psi_{\mathbf{m}, N}, \psi_{\mathbf{m}^{\prime}, N}\right\rangle & =\delta_{\mathbf{m}}\left(\mathbf{m}^{\prime}\right) & & \text { on } B_{N} \text { for all } \mathbf{m}, \mathbf{m}^{\prime} \in B_{N}^{o}, \\
Q_{N} \psi_{\mathbf{m}, N} & =\lambda_{\mathbf{m}, N} \cdot \psi_{\mathbf{m}, N} & & \text { on } B_{N}, \text { where }
\end{aligned}
$$

$$
\lambda_{\mathbf{m}, N}=\frac{1}{3}\left(\cos \left(\frac{2 \pi\left(m_{1}-m_{2}\right)}{3 N}\right)+\cos \left(\frac{2 \pi\left(m_{2}-m_{3}\right)}{3 N}\right)+\cos \left(\frac{2 \pi\left(m_{3}-m_{1}\right)}{3 N}\right)\right) .
$$

Proof. (a) The boundary of $B_{N}$ consists of the points $k\left(\mathbf{e}_{1}-\mathbf{e}_{3}\right)$ (first part), $k\left(\mathbf{e}_{2}-\mathbf{e}_{3}\right)$ (second part) and $N\left(\mathbf{e}_{1}-\mathbf{e}_{3}\right)+k\left(\mathbf{e}_{2}-\mathbf{e}_{1}\right)=(N-k, k,-N)$ (third part), where $k=$ $0, \ldots, N$.

Let $\tau_{i, j} \in \mathfrak{S}_{3}$ be the transposition of $i$ and $j(i \neq j)$. Then

$$
\begin{aligned}
& \psi_{\mathbf{m}, N}\left(k \mathbf{e}_{i}-k \mathbf{e}_{j}\right) \\
& =\frac{1}{\sqrt{6} N} \sum_{\sigma \in \mathfrak{A}_{3}}\left(\exp \left(\frac{2 \pi \mathfrak{i}}{3 N}\left\langle\mathbf{m}, \sigma\left(k \mathbf{e}_{i}-k \mathbf{e}_{j}\right)\right\rangle\right)-\exp \left(\frac{2 \pi \mathfrak{i}}{3 N}\left\langle\mathbf{m},-\sigma \tau_{i, j}\left(k \mathbf{e}_{i}-k \mathbf{e}_{j}\right)\right\rangle\right)\right) \\
& =0 .
\end{aligned}
$$

Thus, $\psi_{\mathbf{m}, N}=0$ on the first and second parts of $\partial B_{N}$. Regarding the third part, note that for $\mathbf{m} \in \mathbb{A}_{2}$,

$$
\langle\mathbf{m},(N-k, k,-N)\rangle+\left\langle\mathbf{m}, \tau_{1,2}(N-k, k,-N)\right\rangle=\left(m_{1}+m_{2}-2 m_{3}\right) N \equiv 0 \bmod 3 N .
$$

Therefore, pairing each element $\sigma \in \mathfrak{A}_{3}$ with $\sigma \tau_{1,2}$ as in the above sum shows that $\psi_{\mathbf{m}, N}=0$ on the third part of $\partial B_{N}$.

(b) We have by construction

$$
\psi_{\mathbf{m}, N}(\mathbf{k})=\operatorname{sign}(\sigma) \psi_{\mathbf{m}, N}(\operatorname{sign}(\sigma) \sigma \mathbf{k}),
$$

and since each of $\langle\mathbf{m},(N, N,-2 N)\rangle,\langle\mathbf{m},(N,-2 N, N)\rangle$ and $\langle\mathbf{m},(-2 N, N, N)\rangle$, for $\mathbf{m} \in \mathbb{A}_{2}$, is a multiple of $3 N$, we get

$$
\begin{aligned}
\psi_{\mathbf{m}, N}(\mathbf{k}) & =\psi_{\mathbf{m}, N}(\mathbf{k}+(N, N,-2 N))=\psi_{\mathbf{m}, N}(\mathbf{k}+(N,-2 N, N)) \\
& =\psi_{\mathbf{m}, N}(\mathbf{k}+(-2 N, N, N)) .
\end{aligned}
$$


That is, up to a change of sign, $\psi_{\mathbf{m}, N}$ is invariant under the group of motions of $\mathbb{A}_{2}$ generated by the translations $\mathbf{k} \mapsto \mathbf{k}+N\left(\mathbf{e}_{i}+\mathbf{e}_{j}-2 \mathbf{e}_{l}\right)$, where $\{i, j, l\}=\{1,2,3\}$, and the reflections $\mathbf{k} \mapsto-\tau_{i, j} \mathbf{k}$, where $1 \leq i<j \leq 3$. Now $B_{3 N}$ is the union of nine distinct images of $B_{N}$ under elements of that group, and those images meet only at their respective boundaries, where $\psi_{\mathbf{m}, N}=0$. Therefore

$$
\left\langle\psi_{\mathbf{m}, N}, \psi_{\mathbf{m}^{\prime}, N}\right\rangle_{B_{3 N}}=9\left\langle\psi_{\mathbf{m}, N}, \psi_{\mathbf{m}^{\prime}, N}\right\rangle_{B_{N}},
$$

where the inner products are taken over $B_{3 N}$ and $B_{N}$, respectively. Also, $\psi_{\mathbf{m}, N}=0$ on $\partial B_{3 N}$. Recall that $\sum_{k=0}^{3 N-1} \exp \left(\frac{2 \pi \mathfrak{i}}{3 N} r k\right)=0$ when $r \in \mathbb{Z}$ is not a multiple of $3 N$. We now obtain, setting $\mathbf{k}=\left(k_{1}, k_{2},-k_{1}-k_{2}\right)$ if $k_{1}$ and $k_{2}$ are given,

$\left\langle\psi_{\mathbf{m}, N}, \psi_{\mathbf{m}^{\prime}, N}\right\rangle_{B_{N}}$

$$
\begin{aligned}
& =\frac{1}{54 N^{2}} \sum_{\sigma, \tau \in \mathfrak{S}} \operatorname{sign}(\sigma \tau) \sum_{k_{1}, k_{2}=0}^{3 N-1} \exp \left(\frac{2 \pi \mathfrak{i}}{3 N}\left\langle\operatorname{sign}(\sigma) \sigma^{-1} \mathbf{m}-\operatorname{sign}(\tau) \tau^{-1} \mathbf{m}^{\prime}, \mathbf{k}\right\rangle\right) \\
& =\frac{1}{6} \sum_{\sigma, \tau \in \mathfrak{S}} \operatorname{sign}\left(\sigma^{-1} \tau\right) \delta_{\mathbf{m}}\left(\operatorname{sign}\left(\sigma^{-1} \tau\right) \sigma \tau^{-1} \mathbf{m}^{\prime}\right) \\
& =\sum_{\sigma \in \mathfrak{S}} \operatorname{sign}(\sigma) \delta_{\mathbf{m}}\left(\left(\operatorname{sign}(\sigma) \sigma \mathbf{m}^{\prime}\right)=\delta_{\mathbf{m}}\left(\mathbf{m}^{\prime}\right),\right.
\end{aligned}
$$

as $B_{N}^{o} \cap(\operatorname{sign}(\sigma) \sigma) B_{N}^{o}=\emptyset$ for $\sigma \neq \mathrm{id}$.

(c) is a straightforward computation.

(5.22) Corollary. The spectrum of the SRW operator $P$ on $\operatorname{DL}(q, q, q)$ is the interval $[-1 / 2,1]$. It is the closure of the set of eigenvalues

$$
\left\{\lambda_{\mathbf{m}, N}: N \geq 2, \mathbf{m} \in \mathbb{A}_{2} \text { with } m_{1}, m_{2}>0, m_{3}>-N\right\}
$$

given by Proposition 5.21.(c).

The associated orthonormal basis of finitely supported eigenfunctions can be computed explicitly by use of the functions $\psi_{\mathbf{m}, N}$ of 5.20$)$, according to Theorem 5.18

We remark here that the groups $\Gamma_{3}\left(\mathfrak{Z}_{q}\right)$ and $\Gamma_{d}\left(\mathbb{F}_{q}\right)(d \geq 3)$ constructed in Corollaries 3.15 and 3.17 are the first examples of finitely presented groups where SRW has a pure point spectrum.

\section{Spectral measure and return probabilities on $\operatorname{DL}(q, q, q)$}

If $\mathfrak{B}$ is the orthonormal basis of Theorem 5.18 and we write $\lambda(g)$ for the eigenvalue associated with $g \in \mathfrak{B}$, then we see that the $n$-step transition probabilities $p^{(n)}(x, y)=$ $p^{(n)}(y, x)=\left\langle P^{n} \delta_{x}, \delta_{y}\right\rangle$ satisfy

$$
\begin{aligned}
p^{(n)}(x, y) & =\sum_{g \in \mathfrak{B}} \lambda(g)^{n} g(x) g(y)=\sum_{\lambda \in \operatorname{spec}_{p}(P)} \lambda^{n} \mu_{x, y}(\lambda), \text { where } \\
\mu_{x, y}(\lambda) & =\sum_{g \in \mathfrak{B}: \lambda(g)=\lambda} g(x) g(y) .
\end{aligned}
$$


Thus, we obtain the $(x, y)$-element $\mu_{x, y}$ of the spectral measure of the self-adjoint operator $P$ (see e.g. Grigorchuk and Żuk [29] for spectra of Markov operators on graphs, and [3] in the context of DL-graphs). Since $\operatorname{spec}(P)$ is pure point, $\mu_{x, y}$ is an infinite sum of weighted point masses $\mu_{x, y}(\lambda) \delta_{\lambda}$. We want to have a closer look at the measure $\mu_{x, x}=\mu_{o, o}$, which plays the role of the Plancherel measure.

The eigenvalues found in Proposition 5.21 satisfy the obvious relation $\lambda_{\mathbf{m}, N}=\lambda_{\ell \mathbf{m}, \ell N}$ for each $\ell \in \mathbb{N}$. Besides, we also have $\lambda_{\mathbf{m}, N}=\lambda_{\tau_{1,2} \mathbf{m}, N}$ (exchanging of $m_{1}$ and $m_{2}$ ), and when $N$ and $\mathbf{m} \in B_{N}^{o}$ are such that $2 m_{1}+4 m_{2}=3 N$ then $\lambda_{\mathbf{m}, N}=-1 / 3$. In general, it is a very delicate task to determine all $\left(\mathbf{m}^{\prime}, N^{\prime}\right)$ for which $\lambda_{\mathbf{m}^{\prime}, N^{\prime}}=\lambda_{\mathbf{m}, N}$, where $(\mathbf{m}, N)$ is given. Thus, instead of a complete description, we pull back $\mu_{o, o}$ to a measure $\tilde{\mu}$ on the parameter space

$$
\mathcal{M}=\left\{(\mathbf{m}, N): N \geq 2, \mathbf{m} \in B_{N}^{o}, \operatorname{gcd}\left(N, m_{1}, m_{2}\right)=1\right\},
$$

by considering only the first, obvious relation above. (Since $m_{3}=-m_{2}-m_{1}$, the parametrization is in reality over all $N \geq 2$ and $m_{1}, m_{2} \geq 1$ with $m_{1}+m_{2} \leq N-1$.) We note that for any polyhedron $\mathrm{S}$, we have $g_{\mathbf{m}}\left[\widetilde{\mathrm{S}}, \varphi_{l_{1}}^{1}, \varphi_{l_{2}}^{2}, \varphi_{l_{3}}^{3}\right](o) \neq 0$ if and only if $o \in S^{o}$ and (since the edge from each $o_{j}$ to its predecessor in $T_{j}$ has label 0 ) if $l_{1}=l_{2}=l_{3}=1$. We write $g_{\mathbf{m}}[\widetilde{\mathbf{S}}]=g_{\mathbf{m}}\left[\widetilde{\mathbf{S}}, \varphi_{1}^{1}, \varphi_{1}^{2}, \varphi_{1}^{3}\right]$. Thus, for $(\mathbf{m}, N) \in \mathcal{M}$, we compute

$$
\tilde{\mu}(\mathbf{m}, N)=\sum_{\ell=1}^{\infty} \mathrm{Z}(\ell \mathbf{m}, \ell N), \quad \text { where } \mathrm{Z}(\mathbf{m}, N)=\sum_{\mathrm{S}: o \in \mathrm{S}^{o}, N(\mathrm{~S})=N}\left(g_{\mathbf{m}}[\tilde{\mathrm{S}}](o)\right)^{2} .
$$

Now let $\mathrm{S}=\mathrm{S}\left(a_{1}, a_{2}, a_{3}\right), o \in \mathrm{S}^{o}, N(\mathrm{~S})=N$ and $\mathbf{h}=\mathbf{h}(\mathrm{S})$. Then $a_{j} \preccurlyeq o_{j}, a_{j} \neq o_{j}$ $(j=1,2,3)$, and $\mathbf{k}=-\mathbf{h}-N \mathbf{e}_{3} \in B_{N}^{o}$. From Corollary 5.14 we get

$$
g_{\mathbf{m}}[\tilde{\mathrm{S}}](o)=\psi_{\mathbf{m}, N}(\mathbf{k}) f_{\mathbf{0}}(o)=\psi_{\mathbf{m}, N}(\mathbf{k})(q-1)^{3 / 2} q^{-N / 2} .
$$

If we vary $\mathrm{S}$ in such a way that $N(\mathrm{~S})=N$ and $o \in \mathrm{S}^{o}$, then this amounts to varying $\mathbf{k}=-\mathbf{h}(\mathrm{S})-N \mathbf{e}_{3}$ in $B_{N}^{o}$. Thus

$$
\mathbf{Z}(\mathbf{m}, N)=\sum_{\mathbf{k} \in B_{N}^{o}} \psi_{\mathbf{m}, N}(\mathbf{k})^{2}(q-1)^{3} q^{-N}=(q-1)^{3} q^{-N},
$$

and therefore

$$
\tilde{\mu}(\mathbf{m}, N)=(q-1)^{3} /\left(q^{N}-1\right) .
$$

The Plancherel measure $\mu_{o, o}$ is the image of $\tilde{\mu}$ under the mapping $\mathcal{M} \rightarrow \operatorname{spec}(P)$, ( $\mathbf{m}, N) \mapsto \lambda_{\mathbf{m}, N}$. (If $m_{1} \neq m_{2}$ then this mapping is at least two-to-one.) We get in particular the following nice expression for the $n$-step return probabilities.

(5.26) Corollary. With $\mathcal{M}$ and $\lambda_{\mathbf{m}, N}$ defined as in 5.24) and Proposition 5.21 respectively, the n-step return probabilities for $\operatorname{SRW}$ on $\mathrm{DL}(q, q, q)$ are given by

$$
\begin{aligned}
p^{(n)}(x, x) & =\sum_{(\mathbf{m}, N) \in \mathcal{M}} \lambda_{\mathbf{m}, N}^{n}(q-1)^{3} /\left(q^{N}-1\right) \\
& \sim A n^{1 / 6} \exp \left(-B n^{1 / 3}\right) \quad \text { as } n \rightarrow \infty, \quad \text { for some } A, B>0 .
\end{aligned}
$$

Here $a_{n} \sim b_{n}$ means that $a_{n} / b_{n} \rightarrow 1$ as $n \rightarrow \infty$. 
We omit the computational details of the asymptotic formula, which follow the method of [3, §5]. Since for $d \geq 4$, the precise information about the spectral measure is not available, we cannot extend the above corollary to all DL-graphs. However, a rougher estimate is available. If $\left(a_{n}\right)$ and $\left(b_{n}\right)$ are two sequences of positive numbers, then we write $a_{n} \preccurlyeq b_{n}$ if there are constants $C, D>0$ such that $a_{n} \leq C b_{D n}$. If $a_{n} \preccurlyeq b_{n}$ and $b_{n} \preccurlyeq a_{n}$, then we say that $\left(a_{n}\right)$ and $\left(b_{n}\right)$ have the same asymptotic type, and write $a_{n} \approx b_{n}$. Thus, SRW on $\operatorname{DL}(q, q, q)$ satisfies $p^{(n)}(x, x) \approx \exp \left(-n^{1 / 3}\right)$ by the above, and the same is true for $\operatorname{DL}(q, q)$ (when $n$ is even, since $\operatorname{DL}(q, q)$ is bipartite); see [6, 3]. Also, for arbitrary $d$, when $q_{1}, \ldots, q_{d}$ do not all coincide then $\rho(P)<1$, that is,

$$
p^{(n)}(x, x) \approx \exp (-n) \quad \text { on } \operatorname{DL}\left(q_{1}, \ldots, q_{d}\right)
$$

again considering only even $n$ when $d=2$.

(5.27) Proposition. For each $d \geq 3$, SRW on $\mathrm{DL}_{d}(q)$ satisfies $p^{(n)}(x, x) \approx \exp \left(-n^{1 / 3}\right)$.

Proof. We follow a well-known method (see e.g. [45, §14-15]). It is a general fact that on a vertex-transitive graph with exponential growth, $p^{(n)}(x, x) \preccurlyeq \exp \left(-n^{1 / 3}\right)$ (see [45, Corollary $14.5(b)]$ ). Thus, we only need to prove the lower bound. This is based on the following estimate, where we take $x=o$, and $A_{m}$ is a suitably chosen finite set of vertices of our graph (see e.g. [45, §15]).

$$
p^{(2 n)}(o, o) \geq \operatorname{Pr}_{o}\left[Z_{n} \in A_{m}\right]^{2} /\left|A_{m}\right|
$$

where $Z_{n}$ is the random position of the random walk starting at $o$. (For details of the probabilistic notions, see $\$ 6$ below.)

Now let $a_{j}=o_{j}^{-m}$ be the $m$-th predecessor of $o_{j}$, and set $A_{m}=\mathrm{S}\left(a_{1}, \ldots, a_{d}\right)$. The cardinality of this set is of the order of $C m^{d-1} q^{d m}$. Let $Z_{j, n} \in T_{j}$ be the $j$-th coordinate of $Z_{n}$. If $Z_{n} \notin A_{m}$ then there must be $k \leq n$ and $j \in\{1, \ldots, d\}$ such that $\mathfrak{h}\left(Z_{j, k}\right)<-m$. Thus, if we set

$$
S_{n}=\max \left\{\left|\mathfrak{h}\left(Z_{j, k}\right)\right|: j=1, \ldots, d, k \leq n\right\},
$$

then $\operatorname{Pr}_{o}\left[Z_{n} \in A_{m}\right] \geq \operatorname{Pr}_{o}\left[S_{n} \leq m\right]$. Since $\mathfrak{H}\left(Z_{n}\right)=\left(\mathfrak{h}\left(Z_{1, n}\right), \ldots, \mathfrak{h}\left(Z_{d, n}\right)\right)$ defines a translation invariant random walk on $\mathbb{A}_{d-1} \cong \mathbb{Z}^{d-1}$, Lemma 1.2 of Alexopoulos [1] implies that there are constants $C^{\prime}, D^{\prime}>0$ such that

$$
\operatorname{Pr}_{o}\left[S_{n} \leq m\right] \geq C^{\prime} \exp \left(-D^{\prime} n / m^{2}\right)
$$

Therefore

$$
p^{(2 n)}(o, o) \geq \widetilde{C} \exp \left(-\widetilde{D}\left(n / m^{2}+m+\log m\right)\right) .
$$

Setting $m=\left\lfloor n^{1 / 3}\right\rfloor$, we obtain the claimed lower estimate. 


\section{The Poisson boundary of a random walk}

In this section, we consider a larger class of random walks than those of $\$ 5$. On DL $=$ $\operatorname{DL}\left(q_{1}, \ldots, q_{d}\right)$, consider a stochastic transition matrix

$$
P=(p(x, y))_{x, y \in \mathrm{DL}}
$$

with the following properties:

(i) Irreducibility: For each $x, y \in \mathrm{DL}$ there is $N$ such that the $(x, y)$-element of $P^{N}$ satisfies $p^{(N)}(x, y)>0$.

(ii) Group-invariance: the group

$$
\Gamma=\{g \in \mathcal{A}: p(g x, g y)=p(x, y) \text { for all } x, y \in \mathrm{DL}\}
$$

acts transitively on DL, where $\mathcal{A}$ is as in Proposition 2.5 .

(iii) Finite first moment: for the graph metric $d(\cdot, \cdot)$ of $\mathrm{DL}$,

$$
m_{1}(P)=\sum_{x} p(o, x) d(o, x)<\infty .
$$

Let $\left(Z_{n}\right)_{n \geq 0}$ be the random walk on $\mathrm{DL}$ governed by $P$, and let $\operatorname{Pr}_{x_{0}}$ be the probability measure on the associated trajectory space for the initial point $Z_{0}=x_{0}$. Thus,

$$
\operatorname{Pr}_{x_{0}}\left[Z_{k+n}=y \mid Z_{k}=x\right]=p^{(n)}(x, y)
$$

whenever $\operatorname{Pr}_{x_{0}}\left[Z_{k}=x\right]=p^{(k)}\left(x_{0}, x\right)>0$.

Since DL is a vertex-transitive graph with exponential growth, the random walk is transient, that is,

$$
\operatorname{Pr}_{x}\left[d\left(Z_{n}, Z_{0}\right) \rightarrow \infty\right]=1
$$

(see Woess [45, Theorem 5.13]). Our first question is whether one can give a more precise geometric description of how $\left(Z_{n}\right)$ tends to infinity in $\mathrm{DL}$.

(6.1) Definition. The geometric compactification $\widehat{\mathrm{DL}}$ of $\mathrm{DL}$ is the closure of $\mathrm{DL}$ in the product $\prod_{j=1}^{d} \widehat{T}_{j}$ of the end compactifications. The geometric boundary of $\mathrm{DL}$ is

$$
\partial \mathrm{DL}=\widehat{\mathrm{DL}} \backslash \mathrm{DL}=\left\{\zeta=\zeta_{1} \cdots \zeta_{d} \in \prod_{j=1}^{d} \widehat{T}_{j}: \zeta_{j}=\omega_{j} \text { for at least one } j\right\} .
$$

To justify the last fact, first observe that $\left(\prod_{j=1}^{d} \widehat{T}_{j}\right) \backslash\left(\prod_{j=1}^{d} T_{j}\right)$ consists of all $\zeta=$ $\zeta_{1} \cdots \zeta_{d} \in \prod_{j=1}^{d} \widehat{T}_{j}$ such that $\zeta_{j} \in \partial T_{j}$ for at least one $j$ (while it may well be that some $\zeta_{i}$ are vertices in $T_{i}$ ). Now, if such a $\zeta$ is the limit of a sequence $x_{n}=x_{1, n} \cdots x_{d, n} \in \mathrm{DL}$, then we can distinguish the following cases:

(1) If there are coordinates $i$ such that $\left|\mathfrak{h}\left(x_{i, n}\right)\right| \rightarrow \infty$ then there must be some $j$ such that $\mathfrak{h}\left(x_{j, n}\right) \rightarrow-\infty$ and consequently $x_{j, n} \rightarrow \omega_{j}$. 
(2) If $\left|\mathfrak{h}\left(x_{i, n}\right)\right|$ is bounded for each $i$, then there still must be some coordinate $j$ such that $d\left(o_{j}, x_{j, n}\right) \rightarrow \infty$. But in this case, we must also have $\mathfrak{u}\left(o_{j}, x_{j, n}\right) \rightarrow \infty$ (see $\left.\sqrt{2.2}\right)$ ). Therefore $x_{j, n} \rightarrow \omega_{j}$.

In general, up to a permutation of the coordinates, the situation will be as follows: there are indices $1 \leq r \leq s \leq d$ such that $\zeta_{j}=\omega_{j}$ for $1 \leq j \leq r$ (and $\mathfrak{h}\left(x_{j, n}\right)$ may be bounded or unbounded from below as well as from above!), $\zeta_{j} \in \partial^{*} T_{j}$ for $r<j \leq s$ (and $\mathfrak{h}\left(x_{j, n}\right) \rightarrow \infty$ ), and $\zeta_{j} \in T_{j}$ for $s<j \leq d$ (and $x_{j, n}=\zeta_{j}$ for all but finitely many $n$ ).

For a detailed description of boundary and convergence in the case $d=2$ (two trees), see [14, (5.3)] or Bertacchi [6].

For $j \in\{1, \ldots, d\}$, let $\Gamma_{j}$ be the image of $\Gamma$ under the projection $\mathcal{A} \rightarrow \operatorname{Aff}\left(T_{j}\right)$. This group acts transitively on $T_{j}$. Write $Z_{j, n}$ for the image of $Z_{n}$ under the projection $\mathrm{DL} \rightarrow T_{j}$. Then $\left(Z_{j, n}\right)$ is an irreducible random walk on $T_{j}$ whose transition probabilities $p_{j}\left(x_{j}, y_{j}\right)$ are $\Gamma_{j}$-invariant. We also consider the image

$$
\mathfrak{H}\left(Z_{n}\right)=\left(\mathfrak{h}\left(Z_{1, n}\right), \ldots, \mathfrak{h}\left(Z_{d, n}\right)\right) \in \mathbb{A}_{d-1} .
$$

This defines a random walk on $\mathbb{A}_{d-1}$ whose transition probabilities are invariant under translation by elements of $\mathbb{A}_{d-1}$. In the same way, $\left(\mathfrak{h}\left(Z_{j, n}\right)\right)_{n \geq 0}$ is a translation invariant random walk on $\mathbb{Z}$. Its increments are i.i.d. integer random variables with expected value

$$
\alpha_{j}=\alpha_{j}(P)=E_{o}\left(\mathfrak{h}\left(Z_{j, 1}\right)\right)=\sum_{x \in \mathrm{DL}} p(o, x) \mathfrak{h}\left(x_{j}\right)
$$

which is finite, since $\left|\alpha_{j}\right| \leq m_{1}(P)$. Note that $\sum_{j} \alpha_{j}=0$, so that there must be a $j$ with $\alpha_{j} \leq 0$. Applying the results of [19] and (for convergence to the boundary when $\alpha_{j}=0$ ) Brofferio [12, Theorem 3.1], one finds the following under the above assumptions (i)-(iii).

(6.2) Proposition. For each $j \in\{1, \ldots, d\}$, we have for the distance on $T_{j}$,

$$
\lim _{n \rightarrow \infty} \frac{d\left(Z_{j, n}, Z_{0, n}\right)}{n}=\left|\alpha_{j}\right| \quad \operatorname{Pr}_{x} \text {-almost surely, }
$$

and there is a $\partial T_{j}$-valued random variable $Z_{j, \infty}$ such that

$$
\lim _{n \rightarrow \infty} Z_{j, n}=Z_{j, \infty} \quad \operatorname{Pr}_{x} \text {-almost surely }
$$

in the topology of $\widehat{T}$, for every $x \in \mathrm{DL}$. One has the following.

(a) If $\alpha_{j} \leq 0$ then $Z_{j, \infty}=\omega_{j}$ almost surely.

(b) If $\alpha_{j}>0$ then $Z_{j, \infty}$ is a $\partial^{*} T_{j}$-valued random variable whose $\operatorname{Pr}_{x}$-distribution is $a$ continuous measure (i.e., it carries no point mass) supported by the whole of $\partial^{*} T_{j}$.

Proof. The results in [19] and [12] are formulated for random walks on Aff $\left(\mathbb{T}_{q}\right)$, that is, for $R_{n} o$, where $R_{n}=g_{0} X_{1} \cdots X_{n}$ (group product) and the $X_{n}$ are i.i.d. group-valued random variables. The only point that we have to clarify is that our situation arises as a special case of that form. 
There is a standard method for turning a $\Gamma$-invariant random walk on a transitive graph into $R_{n} o$, where $\left(R_{n}\right)$ is a random walk on $\Gamma$; see e.g. Kaimanovich and Woess [32, Prop. $2.15]$. Indeed, if $d g$ is the left Haar measure on the (locally compact, totally disconnected) group $\Gamma$, then

$$
\mu(d g)=p(o, g o) d g
$$

defines a probability measure on $\Gamma$. Now let $\left(X_{n}\right)_{n \geq 1}$ be i.i.d. $\mu$-distributed in $\Gamma$. Then $Z_{n}=g_{0} X_{1} \cdots X_{n} o$ defines a Markov chain on the graph with $Z_{0}=g_{0} o$ and transition matrix $P$.

Let us write $\Lambda_{j}\left(\alpha_{j}\right)=\left\{\omega_{j}\right\}$ if $\alpha_{j} \leq 0$, and $\Lambda_{j}\left(\alpha_{j}\right)=\partial^{*} T_{j}$ if $\alpha_{j}>0$, and define the following Borel subset of $\partial \mathrm{DL}$ :

$$
\Lambda=\prod_{j=1}^{d} \Lambda_{j}\left(\alpha_{j}\right)
$$

(6.5) Corollary. Under assumptions (i)-(iii), there is a $\Lambda$-valued random variable $Z_{\infty}$ such that

$$
\lim _{n \rightarrow \infty} Z_{n}=Z_{\infty} \quad \operatorname{Pr}_{x} \text {-almost surely }
$$

in the topology of $\widehat{\mathrm{DL}}$, for every $x \in \mathrm{DL}$. The $\operatorname{Pr}_{x}$-distribution $v_{x}$ of $Z_{\infty}$ is a Borel probability measure supported by the whole of $\Lambda$. It is a continuous measure unless $\Lambda=\left\{\omega_{1} \cdots \omega_{d}\right\}$ is trivial, which happens precisely when $\alpha_{j}=0$ for all $j$.

The measures $v_{x}$ satisfy $v_{x}=\sum_{y} p(x, y) v_{y}$ and are mutually absolutely continuous.

(The last statement is immediate by factoring through the first step of the random walk, and using irreducibility.)

Thus, we see that the space $\Lambda$ together with the family of harmonic measures $\nu_{\bullet}=$ $\left(v_{x}\right)_{x \in \mathrm{DL}}$ is a boundary of the random walk $\left(Z_{n}\right)$ in the sense of [32], a notion going back to Furstenberg [28]. Indeed, the group $\Gamma$ defined in (ii) acts on $\Lambda$, i.e., $\Lambda$ is a $\Gamma$-space, and we have the convolution identity $\mu * v_{o}=v_{o}$ for the probability measure $\mu$ on $\Gamma$ defined in 6.3. This identity holds since $\delta_{g} * v_{o}=v_{g o}$ for every $g \in \Gamma$.

We now want to decide whether this is the "best" (biggest) model, as a measure space, for distinguishing limit points at infinity of our random walk. To formulate this question more precisely, we recall that a $P$-harmonic function is a function $h: \mathrm{DL} \rightarrow \mathbb{R}$ such that $h(x)=\sum_{y} p(x, y) h(y)$. Now, if $\varphi \in L^{\infty}\left(\Lambda, v_{\bullet}\right)$, then

$$
h(x)=\int_{\Lambda} \varphi d v_{x}
$$

defines a mapping from $L^{\infty}\left(\Lambda, v_{\bullet}\right)$ to the space of all bounded harmonic functions. When the mapping is bijective, $\left(\Lambda, \nu_{\bullet}\right)$ is called the Poisson boundary of the random walk. This boundary is unique up to isomorphism of measure spaces. For precise details in the present setting of a group-invariant random walk on a transitive graph, we refer to [32]; additional information can be found in the references given there. The following theorem 
should be compared with the main result (based on a different method) of Brofferio [13], who considers the Poisson boundary of random walks on finitely generated groups of affine mappings with rational coefficients: those groups act on a "weighted" horocyclic product of distinct trees with hyperbolic upper half-plane.

(6.6) Theorem. Under assumptions (i)-(iii), $\left(\Lambda, v_{\bullet}\right)$ is the Poisson boundary of the random walk $\left(Z_{n}\right)$ on $\mathrm{DL}$.

Proof. For the proof, we apply the ray criterion of Kaimanovich (see [32, Thm. 5.18]). Formulated in our specific terms of $\Lambda$ and DL, it says the following.

Suppose that $\Lambda$ is a $\Gamma$-space carrying a family of probability measures $v_{\bullet}=\left(v_{x}\right)_{x \in \mathrm{DL}}$ such that $\delta_{g} * v_{o}=v_{g o}$ and $v_{o}=\sum_{x} p(o, x) v_{x}$. Then $\left(\Lambda, v_{\bullet}\right)$ is the Poisson boundary of the random walk $\left(Z_{n}\right)$, provided that there is a sequence of measurable mappings $\Pi_{n}: \Lambda \rightarrow \mathrm{DL}$ such that, in the graph metric of DL,

$$
\lim _{n \rightarrow \infty} \frac{1}{n} d\left(Z_{n}, \Pi_{n}\left(Z_{\infty}\right)\right)=0 \quad \operatorname{Pr}_{o} \text {-almost surely. }
$$

We now construct $\Pi_{n}$. We know that $\alpha_{j} \leq 0$ for some $j$, and assume without loss of generality that $\alpha_{d} \leq 0$. Let now $\xi=\xi_{1} \cdots \xi_{d} \in \Lambda$ be given, so that $\xi_{j} \in \partial T_{j}$ for each $j$, and $\xi_{j}=\omega_{j}$ for at least one $j$. By Proposition 6.2, $\xi_{d}=\omega_{d}$.

For integer $k \geq 0$, denote by $\xi_{j}(k)$ the element on the geodesic $\overline{o_{j} \xi_{j}}$ at distance $k$ from $o_{j}$. Similarly, if $\xi_{j} \in \partial^{*} T_{j}$ and $k \geq 0$, denote by $\xi_{j}[k]$ the element on the geodesic $\overline{o_{j} \xi_{j}}$ which lies on the horocycle $H_{k}^{j}$ of $T_{j}$ and, in case there are two such elements (only when $k=0$ ), is furthest possible from $o_{j}$. Finally, if $\xi_{j}=\omega_{j}$ and $k \leq 0$ then the geodesic $\overline{o_{j}} \xi_{j}$ intersects $H_{k}$ at the point $\xi_{j}[k]=\omega_{j}(|k|)$. See Figure 8.

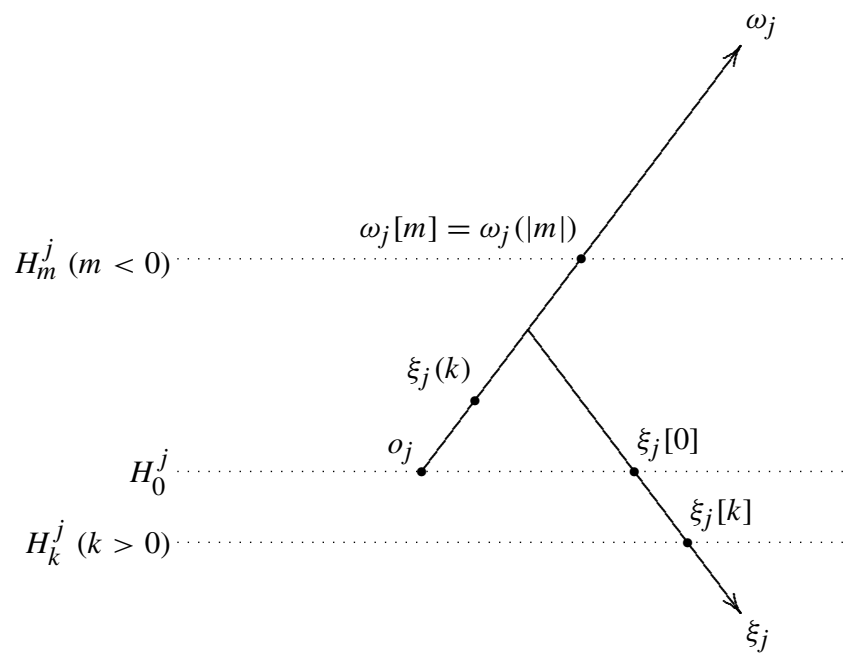

Fig. 8 
Note that

$$
d\left(\xi_{j}[k], \xi_{j}(|k|)\right)=d\left(o_{j}, \xi_{j}[0]\right)
$$

We define

$$
\begin{gathered}
\Pi_{n}(\xi)=x_{1} \cdots x_{d}, \quad \text { where } \quad x_{j}=\Pi_{j, n}\left(\xi_{j}\right)=\xi_{j}\left[k_{j}\right] \text { with } \\
k_{j}=\left\lceil\alpha_{j} n\right\rceil, \quad j=1, \ldots, d-1, \quad \text { and } \quad k_{d}=-k_{1}-\cdots-k_{d-1} .
\end{gathered}
$$

Note that this is well defined: when $\alpha_{j}>0$ we have $\xi_{j} \in \partial^{*} T_{j}$ and $k_{j}>0$, so that $H_{k_{j}}^{j}$ intersects $\overline{o_{j} \xi_{j}}$ in a unique point. When $j<d$ and $\alpha_{j} \leq 0$ we have $\xi_{j}=\omega_{j}$ and $k_{j} \leq 0$, so that $H_{k_{j}}^{j}$ also intersects $\overline{o_{j} \omega_{j}}$ in a unique point. This also holds for $j=d$, since $k_{d} \leq-\alpha_{1} n-\cdots-\alpha_{d-1} n=\alpha_{d} n \leq 0$.

We now apply the results and methods of [19]. Since $m_{1}(P)<\infty$, we also have $m_{1}\left(P_{j}\right)<\infty$, where $P_{j}$ is the transition matrix of $\left(Z_{j, n}\right)_{n \geq 0}$ on $T_{j}$. This implies via the law of large numbers that

$$
\frac{1}{n} d\left(Z_{j, n+1}, Z_{j, n}\right) \rightarrow 0 \quad \text { and } \quad \frac{1}{n} \mathfrak{h}\left(Z_{j, n}\right) \rightarrow \alpha_{j} \quad \operatorname{Pr}_{o} \text {-almost surely. }
$$

Applying [19, Def. 1 and Prop. 1], one finds that $\left(Z_{j, n}\right)_{n}$ is almost surely a regular sequence in $T_{j}$, that is,

$$
\frac{1}{n} d\left(Z_{j, n}, Z_{j, \infty}\left(\left\lceil\alpha_{j} n\right\rceil\right)\right) \rightarrow 0 \quad \operatorname{Pr}_{o} \text {-almost surely. }
$$

Now 6.7) implies that in the metric of $T_{j}$ also

$$
\frac{1}{n} d\left(Z_{j, n}, \Pi_{j, n}\left(Z_{j, \infty}\right)\right) \rightarrow 0 \quad \operatorname{Pr}_{o} \text {-almost surely. }
$$

Since for arbitrary $x, y \in \mathrm{DL}$ we have $d(x, y) \leq \sum_{j=1}^{d} d\left(x_{j}, y_{j}\right)$, we conclude that in the metric of DL,

$$
\frac{1}{n} d\left(Z_{n}, \Pi_{n}\left(Z_{\infty}\right)\right) \rightarrow 0 \quad \operatorname{Pr}_{o} \text {-almost surely, }
$$

so that the criterion applies.

Since $Z_{j, n} \rightarrow \omega_{j}$ almost surely, when $\alpha_{j} \leq 0$, we may omit this part in the description of the Poisson boundary, and can consider the harmonic measures $v_{x}$ as measures on the product of all $\partial^{*} T_{j}$ where $\alpha_{j}>0$.

(6.8) Corollary. The Poisson boundary of the random walk $\left(Z_{n}\right)$ satisfying (i)-(iii) can be identified with the space

$$
\prod_{j: \alpha_{j}>0} \partial^{*} T_{j}
$$

If $\alpha_{j}=0$ for all $j$ then the Poisson boundary is trivial, and all bounded harmonic functions are constant. 
In particular, for SRW on $\operatorname{DL}\left(q_{1}, \ldots, q_{d}\right)$, if we set $\bar{q}=\left(q_{1}+\cdots+q_{d}\right) / d$, then the Poisson boundary can be identified with

$$
\prod_{j: q_{j}>\bar{q}} \partial^{*} T_{j}
$$

it is trivial precisely when all $q_{j}$ coincide.

Indeed, for SRW, we compute $\alpha_{j}=\left(q_{j}-\bar{q}\right) d / D$, where $D$ is the vertex degree in DL.

\section{Outlook}

Here are some further examples of Busemann pairs that are not necessarily trees or treelike.

In some graph-theoretical papers, such graphs are said to have property $Z$, because they admit a graph homomorphism onto the canonical Cayley graph of $\mathbb{Z}$, that is, the two-way-infinite path. See in particular the interesting papers by Cameron, Praeger and Wormald [16], Evans [26], and Malnič, Marušič, Seifter and Zgrablić [34], where digraphs (oriented graphs) are studied whose isometry group acts transitively on the set of oriented paths of length $s$, for every $s$. Of course, in our setting we consider the underlying undirected graph, where the edge orientation is forgotten. Besides the homogeneous trees (with orientation according to the ancestor relation), $\mathrm{DL}\left(q_{1}, q_{2}\right)$ itself provides an example of this type.

If $\Gamma$ is any finitely generated group which admits a group homomorphism onto the additive group $\mathbb{Z}$, then suitable Cayley graphs of $\Gamma$ will have property $Z$, i.e., they will be Busemann pairs. For example, we can take the usual lattice $\mathbb{Z}^{d}$ where two points are neighbours if they are at distance 1 , and define $\mathfrak{h}\left(k_{1}, \ldots, k_{d}\right)=k_{1}+\cdots+k_{d}$.

A more interesting example is the amenable Baumslag-Solitar group $\mathrm{BS}_{q}=\langle a, b|$ $\left.a b=b^{q} a\right\rangle$. It has a natural homomorphism onto the infinite cyclic group generated by $a$, and its Cayley graph with respect to the set of generators $\left\{a^{ \pm 1},(a b)^{ \pm 1}\right\}$ has property Z. Indeed, it is a discretization of the horocyclic product of the hyperbolic plane $\mathbb{H}$ and the tree $\mathbb{T}_{q}$, and fully merits the name of Busemann pair.

The horocyclic product of the hyperbolic plane $\mathbb{H}$ and a tree is called a treebolic space $\mathrm{HT}(q, r)$ in forthcoming work of Bendikov, Saloff-Coste, Salvatori and Woess. Here, one considers the tree $\mathbb{T}_{r}$ as a 1-complex, where each edge is a homomorphic copy of the unit interval, the tree metric is extended in the natural way to the interior of each edge, and the Busemann (horocycle) function with respect to the reference end becomes $\mathbb{R}$-valued. Then $\operatorname{HT}(q, r)$ consists of all pairs $(z, w) \in \mathbb{H} \times \mathbb{T}_{r}$ with $\operatorname{Im} z=q^{\mathfrak{h}(w)}$. This is a 2dimensional complex with a natural metric inherited from $\mathbb{H}$ and $\mathbb{T}_{r}$. The group $\mathrm{BS}_{q}$ acts by isometries and with compact quotient on $\mathrm{HT}(q, q)$ (cf. Farb and Mosher [27]). Treebolic space is another example of a Busemann pair, with real-valued Busemann function.

In a similar way, the construction of horocyclic products of two hyperbolic planes leads to Sol-groups, resp. Sol-manifolds (cf. [25]). They are also Busemann pairs with real-valued Busemann function. 
More generally, one can also consider "Busemann pairs" where the horocycle function $\mathfrak{h}$ takes its values in $\mathbb{Z}^{d}$ (or $\mathbb{R}^{d}$, or any Abelian group). The horocyclic product of two or more spaces of this type (all with the same $d$ ) is then defined in the same way as in (1.1). (This construction has already been suggested in [32, p. 356].) For example, $\tilde{A}_{d}$-buildings have a boundary at infinity and a Busemann (horocycle) function with respect to a given boundary point that is $\mathbb{Z}^{d}$-valued (cf. e.g. Cartwright [18]). The resulting horocyclic products are the object of current investigations by J. Parkinson et al.

Acknowledgments. We acknowledge an email exchange with Brian J. McCartin on the spectrum of the Laplacian on equilateral triangles. We are particularly indebted to Christophe Pittet for an extremely useful discussion about the construction of co-compact lattices in the isometry group of $\mathrm{DL}_{3}(q)$. Fruitful conversations with André Henriques and Christian Wüthrich are gratefully acknowledged.

This research was supported by FWF (Austrian Science Fund) project P15577.

\section{References}

[1] Alexopoulos, G.: A lower estimate for central probabilities on polycyclic groups. Canad. J. Math. 44, 897-910 (1992) Zbl 0762.31003 MR 1186471

[2] Bartholdi, L., Grigorchuk, R. I., Nekrashevych, V.: From fractal groups to fractal sets. In: Fractals in Graz 2001, P. Grabner and W. Woess (eds.), Birkhäuser, Basel, 25-118 (2003) Zbl 1037.20040 MR 2091700

[3] Bartholdi, L., Woess, W.: Spectral computations on lamplighter groups and Diestel-Leader graphs. J. Fourier Anal. Appl. 11, 175-202 (2005) Zbl 1037.20040 MR 2131635

[4] Baumslag, G.: A finitely presented metabelian group with a free abelian derived group of infinite rank. Proc. Amer. Math. Soc. 35, 61-62 (1972) Zbl 0269.20029 MR 0299662

[5] Baumslag, G.: Finitely presented metabelian groups. In: Proc. Second Int. Conference on the Theory of Groups (Canberra, 1973), Lecture Notes in Math. 372, Springer, Berlin, 65-74 (1974) Zbl 0304.20015 MR 0404462

[6] Bertacchi, D.: Random walks on Diestel-Leader graphs. Abh. Math. Sem. Univ. Hamburg 71, 205-224 (2001) Zbl 0992.60052 MR 1873044

[7] Bestvina, M., Brady, N.: Morse theory and finiteness properties of groups. Invent. Math. 129, 445-470 (1997) Zbl 0888.20021 MR 1465330

[8] Bieri, R.: Homological Dimension of Discrete Groups. Queen Mary College Math. Notes, Univ. of London (1976) Zbl 0357.20027 MR 0466344

[9] Bieri, R., Groves, J. R. J.: Metabelian groups of type (FP) $\infty$ are virtually of type (FP). Proc. London Math. Soc. 45, 365-384 (1982) Zbl 0501.20022 MR 0670042

[10] Bogley, W. A., Harlander, J.: Improving tameness for metabelian groups. New York J. Math. 10, 287-294 (2004) Zbl pre02166327 MR 2114792

[11] Bridson, M. R.: Doubles, finiteness properties of groups, and quadratic isoperimetric inequalities, J. Algebra 214, 652-667 (1999) Zbl 0926.20026 MR 1680601

[12] Brofferio, S.: Renewal theory on the affine group of an oriented tree. J. Theoret. Probab. 17, 819-859 (2004) Zbl 1065.60123 MR 2105737

[13] Brofferio, S.: Poisson boundary for finitely generated groups of rational affinities. J. Math. Sci. (N.Y.), to appear

[14] Brofferio, S., Woess, W.: Green kernel estimates and the full Martin boundary for random walks on lamplighter groups and Diestel-Leader graphs. Ann. Inst. H. Poincaré Probab. Statist. 41, 1101-1123 (2005); Erratum, 42, 773-774 (2006) Zbl 1083.60062 
[15] Bux, K.-U.: Finiteness properties of certain metabelian arithmetic groups in the function field case. Proc. London Math. Soc. (3) 75, 308-322 (1997) Zbl 0922.20051 MR 1455858

[16] Cameron, P. J., Praeger, Ch. E., Wormald, N. C.: Infinite highly arc transitive digraphs and universal covering digraphs. Combinatorica 13, 377-396 (1993) Zbl $0793.05065 \mid$ MR 1262915

[17] Cartier, P.: Fonctions harmoniques sur un arbre, Symposia Math. 9, 203-270 (1972) Zbl 0283.31005 MR 0353467

[18] Cartwright, D. I.: Spherical harmonic analysis on buildings of type $\tilde{A}_{n}$. Monatsh. Math. 133, 93-109 (2001) Zbl 1008.51019 MR 1860293

[19] Cartwright, D. I., Kaimanovich, V. A., Woess, W.: Random walks on the affine group of local fields and of homogeneous trees. Ann. Inst. Fourier (Grenoble) 44, 1243-1288 (1994) Zbl 0809.60010 MR 1306556

[20] Cartwright, D. I., Steger, T.: A family of $\tilde{A}_{n}$-groups. Israel J. Math. 103, 125-140 (1998) Zbl 0923.51010 MR 1613560

[21] Cleary, S., Riley, T. R.: A finitely presented group with unbounded dead-end depth. Proc. Amer. Math. Soc. 134, 343-349 (2006) Zbl pre02227172 MR 2176000

[22] Dicks, W., Schick, Th.: The spectral measure of certain elements of the complex group ring of a wreath product. Geom. Dedicata 93, 121-137 (2002) Zbl 1021.47014 MR 1934693

[23] Diestel, R., Leader, I.: A conjecture concerning a limit of non-Cayley graphs. J. Algebraic Combin. 14, 17-25 (2001) Zbl 0985.05020 MR 1856226

[24] Epstein, D. B. A., Cannon, J. W., Holt, D. F., Levy, S. V. F., Paterson, M. S., Thurston, W. P.: Word Processing in Groups, Jones and Bartlett (1992) Zbl 0764.20017 MR 1161694

[25] Eskin, A., Fisher, D., Whyte, K.: Coarse differentiation of quasi-isometries I: spaces not quasiisometric to Cayley graphs. Preprint, Univ. Chicago (2006); arXiv:math.GR/0607207.

[26] Evans, D. M.: An infinite highly arc-transitive digraph. Eur. J. Combin. 18, 281-286 (1997) Zbl 0873.05050 MR 1437003

[27] Farb, B., Mosher, L.: A rigidity theorem for the solvable Baumslag-Solitar groups. With an appendix by Daryl Cooper. Invent. Math. 131, 419-451 (1998) Zbl 0937.22003 MR 1608595

[28] Furstenberg, H.: Random walks and discrete subgroups of Lie groups. In: Advances in Probability and Related Topics, 1, P. Ney (ed.), Dekker, New York. 1-63 (1971) Zbl 0221.22008 MR 0284569

[29] Grigorchuk, R. I., Żuk, A.: The lamplighter group as a group generated by a 2-state automaton, and its spectrum. Geom. Dedicata 87, 209-244 (2001) Zbl 0990.60049||MR 1866850

[30] Gromov, M.: Infinite groups as geometric objects. In: Proc. Int. Congress of Mathematicians (Warszawa, 1983), PWN, Warszawa, 385-392 (1984) Zbl 0599.20041 MR 0804694

[31] Johnson, D. L.: Embedding some recursively presented groups. In: Groups St. Andrews 1997 in Bath, II, London Math. Soc. Lecture Note Ser. 261, Cambridge Univ. Press, Cambridge, 410-416 (1999) Zbl 0923.20025 MR 1676637

[32] Kaimanovich, V. A., Woess, W.: Boundary and entropy of space homogeneous Markov chains. Ann. Probab. 30, 323-363 (2002) Zbl 1021.60056 MR 1894110

[33] Lamé, G: Mémoire sur la propagation de la chaleur dans les polyèdres. J. École Polytechnique 22, 194-251 (1833),

[34] Malnič, A., Marušič, D., Seifter, N., Zgrablić, B.: Highly arc-transitive digraphs with no homomorphism onto $\mathbb{Z}$. Combinatorica 22, 435-443 (2002) Zbl 1012.05080 MR 1932063

[35] Margulis, G. A: Discrete Subgroups of Semisimple Lie Groups. Ergeb. Math. Grenzgeb. (3) 17, Springer, Berlin (1991) Zbl 0732.22008 MR 1090825

[36] McCartin, B. J.: Eigenstructure of the equilateral triangle. I. The Dirichlet problem. SIAM Rev. 45, 267-287 (2003) Zbl pre01982168 MR 2010379 
[37] Paterson, A. L. T.: Amenability. Math. Surveys Monogr. 29, Amer. Math. Soc., Providence, RI (1988) Zbl 0648.43001 MR 0961261

[38] Pinsky, M. A.: Completeness of the eigenfunctions of the equilateral triangle. SIAM J. Math. Anal. 16, 848-851 (1985) Zbl 0593.35032 MR 0793926

[39] Práger, M.: Eigenvalues and eigenfunctions of the Laplace operator on an equilateral triangle. Appl. Math. 43, 311-320 (1998) Zbl 0940.35059 MR 1627985

[40] Práger, M.: Eigenvalues and eigenfunctions of the Laplace operator on an equilateral triangle for the discrete case. Appl. Math. 46, 231-239 (2001) Zbl 1059.65101 MR 1828307

[41] Soardi, P. M., Woess, W.: Amenability, unimodularity, and the spectral radius of random walks on infinite graphs, Math. Z. 205, 471-486 (1990) Zbl 0693.43001 MR 1082868

[42] Stallings, J.: A finitely presented group whose 3-dimensional integral homology is not finitely generated. Amer. J. Math. 85, 541-543 (1963) Zbl 0122.27301 MR 0158917

[43] Trofimov, V. I.: Automorphism groups of graphs as topological groups, Math. Notes 38, $171-$ 720 (1985) Zbl 0596.05033 MR 0811571

[44] Woess, W.: Topological groups and infinite graphs, Discrete Math. 95, 373-384 (1991) Zbl 0757.05060 MR 1141949

[45] Woess, W.: Random Walks on Infinite Graphs and Groups, Cambridge Tracts in Math. 138, Cambridge Univ. Press, Cambridge (2000) Zbl 0951.60002 MR 1743100

[46] Woess, W.: Lamplighters, Diestel-Leader graphs, random walks, and harmonic functions. Combin. Probab. Comput. 14, 415-433 (2005) Zbl 1066.05075 MR 2138121

[47] Wortman, K.: A finitely presented solvable group with a small quasi-isometry group. Michigan Math. J. 55, 3-24 (2007) Zbl pre05212928 MR 2320169 